Florida International University FIU Digital Commons

7-12-2011

\title{
Simulation of Rising Bubbles Dynamics Using the Lattice Boltzmann Method
}

Merlin Ngachin

Florida International University, mngachin@yahoo.com

DOI: $10.25148 /$ etd.FI1 1081007

Follow this and additional works at: https://digitalcommons.fiu.edu/etd

\section{Recommended Citation}

Ngachin, Merlin, "Simulation of Rising Bubbles Dynamics Using the Lattice Boltzmann Method" (2011). FIU Electronic Theses and Dissertations. 466.

https://digitalcommons.fiu.edu/etd/466

This work is brought to you for free and open access by the University Graduate School at FIU Digital Commons. It has been accepted for inclusion in FIU Electronic Theses and Dissertations by an authorized administrator of FIU Digital Commons. For more information, please contact dcc@fiu.edu. 


\title{
FLORIDA INTERNATIONAL UNIVERSITY
}

Miami, Florida

\section{SIMULATION OF RISING BUBBLES DYNAMICS USING THE LATTICE BOLTZMANN METHOD}

\author{
A thesis submitted in partial fulfillment of the \\ requirements for the degree of \\ MASTER OF SCIENCE \\ in \\ GEOSCIENCES \\ by \\ Merlin Ngachin \\ 2011
}


To: Dean Kenneth Furton

College of Arts and Sciences

This thesis, written by Merlin Ngachin, and entitled Simulation of Rising Bubbles Dynamics Using the Lattice Boltzmann Method, having been approved in respect to style and intellectual content, is referred to you for judgment.

We have read this thesis and recommend that it be approved.

Ping Zhu

Michael C. Sukop, Major Professor

Date of defense: July 12, 2011

The thesis of Merlin Ngachin is approved.

Dean Kenneth Furton College of Arts and Sciences

Dean Lakshmi N. Reddi University Graduate School

Florida International University, 2011 


\section{DEDICATION}

This thesis is dedicated to my lovely wife Jacqueline Yhamen for being so supportive and understanding. I have not been able to spend time with you during this long and quite difficult moment you have been through. I hope you find here the love I was unable to share when needed. 


\section{ACKNOWLEDGMENTS}

This work was supported by the US Department of Energy and has been made possible with the help of many people.

The supervision and guidance of my Major Professor, Dr. Michael C. Sukop will never be fully recognized just by few words. He has opened me the door of a new world and has shown me the way by his advices, his patience, and encouragement. Working with him for these past few years has been an honor and a privilege.

I am indebted to my mentor at the Applied Research Center, Dr. Seckin Gokaltun, for being such an inspiration. I will never be able to express my gratitude for being so close to me, and able to answer every question since we began to work together. Your suggestions and guidance have contributed a lot, not only in the realization of this research work, but also in other aspects of my life.

To Dr. Leonel E. Lagos, who has given me this opportunity to enter the US DOE Fellowship program, and to work on several DOE projects. Your advice was valuable and is most appreciated. Find here the expression of my deepest gratitude.

My sincere thanks also go to Dr. Ping Zhu for being part of the Committee and for his valuables comments on the thesis. 
To the CFD team Mr. Rinaldo G. Galdamez, and Mr. Stephen Wood. We share so many things together, and working with you has been so valuable and fruitful.

My final thanks go to all the people I forgot to mention here, and who have been in anyway involved in this research work. 


\title{
ABSTRACT OF THE THESIS \\ SIMULATION OF RISING BUBBLES DYNAMICS USING THE LATTICE \\ BOLTZMANN METHOD
}

\author{
By \\ Merlin Ngachin \\ Florida International University, 2011 \\ Miami, Florida \\ Professor Michael C. Sukop, Major Professor
}

The main purpose of this thesis was to propose and test a new approach that captures the features of single and multiple bubbles dynamics using the Shan and Chen-type lattice Boltzmann method (LBM).

Two dimensional bubbles motions were simulated considering the buoyancy effect for which the topology of the bubble is characterized by the Eötvös $(E o)$, and Morton $(M)$ numbers. A qualitative and quantitative validation were performed using the Level set method. Bubble shape deformation was captured and analysis based on terminal Reynolds number and degree of circularity show very good agreement with the experimental results and with available simulation results. In sum, this study presents crucial preliminary information to further analyze multiphase fluid flows in various contexts. 


\section{TABLE OF CONTENTS}

CHAPTER

PAGE

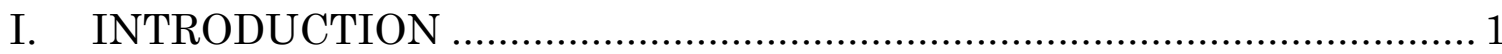

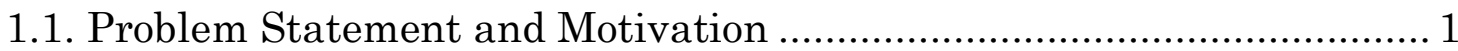

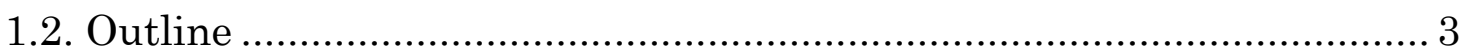

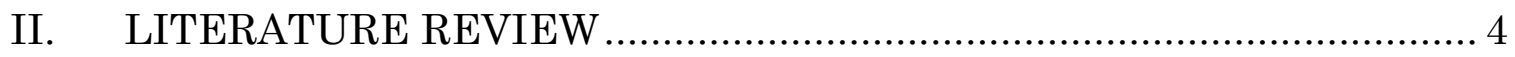

2.1. Experimental Studies and Correlations ............................................... 4

2.2. Multiphase Flow Simulation Using Computational Fluid Dynamics.... 8

2.3. Multiphase Lattice Boltzmann Method................................................ 11



III. LATTICE BOLTZMANN FORMULATION ....................................... 18

3.1. Single Component Lattice Boltzmann Method ................................... 18

3.2. Multicomponent Multiphase Lattice Boltzmann Method .................... 23

3.3. Shan and Chen Interparticle Potential Model .................................... 23

IV. SIMULATION OF SINGLE AND MULTIPLE BUBBLES ................... 27

4.1. Test Case Evaluation of Multicomponent Simulation........................ 27

4.1.1. Laplace Test: Interfacial Tension Evaluation................................ 27

4.1.2. Case Comparison of Single Rising Bubble...................................... 30



4.2.1. Bubble Shape, Terminal Rising Velocity, and Reynolds Number . 38 4.2.2. Wall Effects on Final Shape and Terminal Velocity of Single Rising

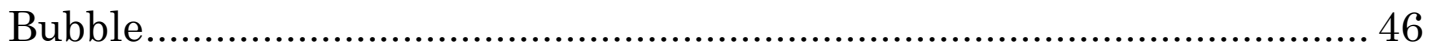


4.2.3. Effects on the Flow Field

4.3. Static Bubble Simulation with Explicit Density Difference................ 57

4.4. Multiple Bubbles Simulation ............................................................ 59

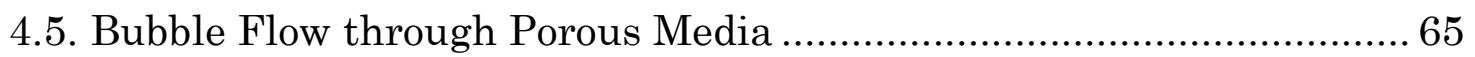



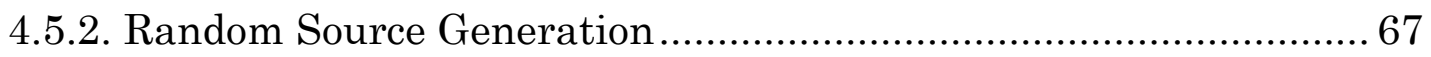

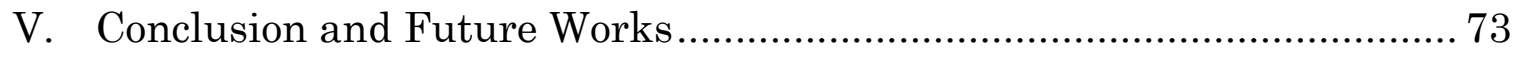

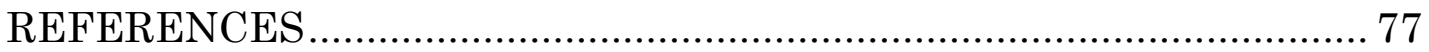

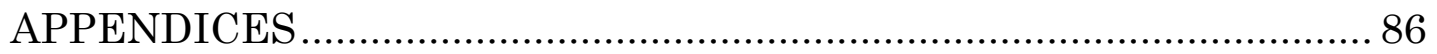




\section{LIST OF FIGURES}

\section{FIGURE}

PAGE

Figure 2.1. Shape regime diagram for bubbles in gravitational motion through liquids. s: spherical; oe: oblate ellipsoid; oed: oblate ellipsoidal (disklike and wobbling); oec: oblate ellipsoidal cap; scc: spherical cap with closed steady wake; sco: spherical cap with open, unsteady wake; sks: skirted with smooth, steady skirt; skw: skirted with wavy, unsteady skirt (adapted from Bhaga and Weber [13])

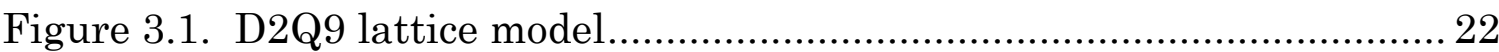

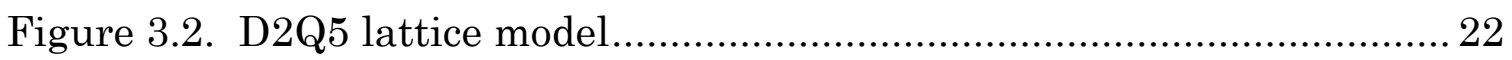

Figure 4.1. Steady density contour and velocity field for a static bubble. The blue bubble of radius $\mathrm{r}=25 \mathrm{lu}$ is surrounded by the red liquid. Domain

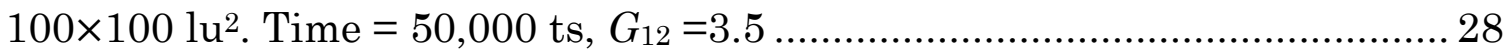

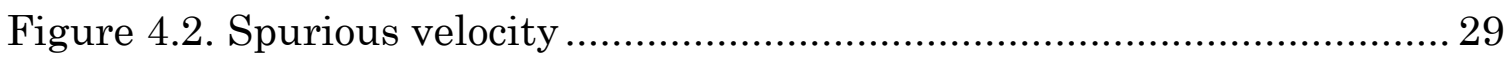

Figure 4.3. Pressure difference between the inside and the outside of a series of bubbles as a function of inverse bubble radius. Gкк $=3.5, \mathrm{t}=$ 50,000 time steps.

Figure 4.4. Representation of the buoyancy force versus the weight

force. $\rho H$ is the heavier liquid density, and $\rho L$ the lighter gas density.

Figure 4.5. Bubble shape at time $\mathrm{T}=4.2$ obtained with the reference method (COMSOL, blue) and the TP2D (red) for Eo $=9$ and $\mathrm{M}=6 \times 10^{-4}$ and a grid size of $50 \times 100$

Figure 4.6. Rise velocity as function of time obtained with the reference method (COMSOL, blue) and the TP2D (red) for $E_{O}=9$ and $M=6 \times 10^{-4}$. The grid sizes are shown in parenthesis.

Figure 4.7. Bubble shape evolution at $E o=9$ and $M=6 \times 10^{-4}$, as it forms an oblate ellipsoidal shape, obtained with LBM (left) in a $107 \times 212$ lu domain and LSM (right) in a $40 \times 80$ domain. The time in this plot is dimensionless $\mathrm{T}=\mathrm{t} /$ tref. 
Figure 4.8. Circularity at $E_{O}=9$ and $M=6 \times 10^{-4}$, obtained with LBM and LSM.

Figure 4.9 : Reynolds number as function of time obtained with LBM and LSM for $E o=9$ and $M=6 \times 10^{-4}$.

Figure 4.10. Bubble shape for $E_{O}=5$ and $M=1.3 \times 10^{-5}$. The red liquid surrounds the blue bubble.

Figure 4.11. Bubble shape evolution for cases $1 \mathrm{a}, 2 \mathrm{~b}$, and $3 \mathrm{~b}$.

Figure 4.12. Interfacial profile of rising bubbles simulated with LBM (top) and LSM (bottom).

Figure 4.13. a) The images in the inset are some results of the numerical simulations using Lattice Boltzmann method; b) Bubble shape regime of my work along with other available results. s: spherical; oe: oblate ellipsoid; oed: oblate ellipsoidal (disk-like and wobbling); oec: oblate ellipsoidal cap; scc: spherical cap with closed steady wake; sco: spherical cap with open, unsteady wake; sks: skirted with smooth, steady skirt; skw: skirted with wavy, unsteady skirt [13]. The dashed line represents the Air/water system obtained with the Fan-Tsuchiya correlation [43].

Figure 4.14. Terminal Reynolds number and circularity simulated with LBM and LSM.

Figure 4.15. Velocity vectors and final shape for ellipsoidal bubbles at $\mathrm{T}=$ $6.30, E O=5$ and $M=9.34 \times 10^{-5}$. The right-hand boundaries represent walls.

Figure 4.16. Wall effects on terminal Reynolds number, $E_{O}=5$, and $M=9.34 \times 10^{-5}$.

Figure 4.17. Wall effects on terminal Reynolds number for case 2a. $E o=5$ and $M=1.3 \times 10^{-5}$.

Figure 4.18. Comparison of spherical cap rise velocity with Krishna-Van Baten model for $\mathrm{r}^{*}=0.166$ and $E o=75 . V *=V t /$ gapplieddo. 51

Figure 4.19. Normalized bubble area obtained with with LBM and LSM..... 52 
Figure 4.20. Grid convergence study for case 5a using LSM.

Figure 4.21. Velocity vectors of the bubble in the domain reference frame, as it forms its terminal shape for different $E_{o}$ and $M$. The case is indicated at the top of the graph, and the associated Eo and $\mathrm{M}$ can be found in Table 1.

Figure 4.22. Streamlines around the bubble in the reference frame at the top of the bubble, at the terminal state for different $E o$ and $M$. The cases are indicated at the bottom of each graph, and the associated $E o$ and $M$ can be found in Table 1.

Figure 4.23. Free deformation of a static bubble from square shape to a circular shape.

Figure 4.24. Coalescence of two identical circular bubbles and free deformation to a circular shape.

Figure 4.25 : Density profile along the centerline of the bubble. The lighter gas is surrounded by the heavy liquid.

Figure 4.26. Bubble shape and velocity fields of two identical bubbles of diameter $23 \mathrm{lu}$. Initially the bubbles are on the same horizontal plane and separated by $3 d o$. Snapshots are taken at $\mathrm{T}=2.10,7.57,13 . E_{o}=1.6$, and $M=2.6 \times 10^{-4}$.

Figure 4.27. Bubble shape and velocity fields of two identical bubbles of diameter $30 \mathrm{lu}$. Initially the bubbles are separated by $3 d o$. Collisions occurs at $\mathrm{T}=8.42$, the coalescence at $\mathrm{T}=8.84$ and bubble attains its terminal ellipsoidal shape at 10.95. Eo $=4$, and $M=5.63 \times 10^{-4}$. 61

Figure 4.28. Bubble shape and velocity fields of two co-axial bubbles in a domain of $120 \times 350$ lattice nodes. Initially, the leading bubble has a diameter of $40 \mathrm{lu}$ and the trailing bubble $30 \mathrm{lu}$. Collision occurs at $\mathrm{T}=7.92$, coalescence at $\mathrm{T}=7.30$. The encapsulated liquid drop is shown at $\mathrm{T}=10.59$. The last snapshot shows a bump at the bottom of the bubble indicating the escape of the liquid drop.

Figure 4.29. Bubble shape and velocity fields of two oblique bubbles in a domain of $174 \times 300$ lattice nodes. $E o=16$ and $M=2 \times 10^{-4}$. The top snapshots represent the LBM results. Bubble diameter $=108 \mathrm{lu}$. From left to right, the 
snapshots correspond to $\mathrm{T}=1.17,2.75$ and 3.35 . The bottom snapshots show results obtained by Annaland et al. [21] in a $80 \times 80 \times 160$ grid size.

Figure 4.30. Line source generation in a multicomponent system and assuming a zero order kinetic. Frames from left to right: 1000, 1500, 8000, 10000,20000 time steps in a $100 \times 100 \mathrm{lu}^{2}$ domain. $\mathrm{g}=1.26 \times 10^{-6}$.

Figure 4.31. Multicomponent random nucleation. Frames from left to right: $1000,1500,2000,10,000$ time steps in a $100 \times 100 \mathrm{lu}^{2}$ domain. $\mathrm{g}=$ $1.26 \times 10^{-5}$. 68

Figure 4.32. Interaction of multicomponent fluids with solid surfaces for different contact angles with fluid 1 (in white). Snapshots represent 20,000 time steps from an initial condition of a $30 \times 30 \mathrm{lu}^{2}$ square of fluid 1 surrounded by fluid 2 (in red) in a $300 \times 50$ lattice nodes domain. Each region is associated with density $\rho A=1$ and a dissolved density $\rho B \sim 10-3$ at equilibrium. Images from left to right and top to bottom: Initial condition, $0^{0}, 15^{0}, 30^{\circ}, 45^{0}, 60^{\circ}, 90^{\circ}, 120^{\circ}, 135^{\circ}$, and $150^{\circ}$

Figure 4.33. Multicomponent fluid flows interacting with solid surfaces for different contact angles. The white bubble is the wetting fluid. Simulation domain: 100×100 lattice nodes. Frames from left to right: 500, 1000, 1500, 2500, and 4000 time steps. From top to bottom: $180^{\circ}, 120^{\circ}, 90^{\circ}$, and $15^{0}$ contact angle. 


\section{LIST OF TABLES}

TABLE

PAGE

Table 1. Parameters used in the Lattice Boltzmann simulations

Table 2. Terminal Reynolds number obtained with LBM and LSM............... 46

Table 3. Parameters used to investigate the wall effects.............................. 47

Table 4. Reynolds numbers and relative difference due to wall effects on single rising bubble, $E o=5$, and $M=9.34 \times 10^{-5}$.

Table 5. Adjusted Reynolds numbers associated with wall effects................ 50

Table 6. Parameters used for the oblique coalescence. ..................................... 63

Table 7. Adhesion parameters and contact angles as obtained with LBM and ImageJ. The last column gives the relative difference between the two

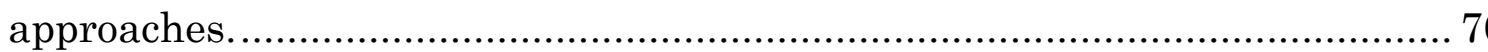




\section{LIST OF ABBREVIATIONS AND SYMBOLS}

\section{NOMENCLATURE}

CFD Computational fluid dynamics

c Lattice constant

$c_{S} \quad$ Speed of son

$d_{o} \quad$ Bubble initial diameter

Eo Eötvös number

$\boldsymbol{e}_{a} \quad$ Discrete velocities

$f \quad$ Particle distribution function

$f^{e q} \quad$ Equilibrium distribution function

g Acceleration due to gravity

$G_{\kappa \bar{\kappa}} \quad$ Interaction strength

LBM Lattice Boltzmann method

lu Lattice units

LSM Level Set Method

M Morton number

$m_{\kappa} \quad$ Molecular mass of component $\kappa$

P Pressure

R Gas constant

Re Reynolds number

T Dimensionless time

$u^{\prime} \quad$ Composite velocity

$\mathbf{u} \quad$ Velocity field $u^{e q} \quad$ Equilibrium velocity

$\mathrm{V} \quad$ Interaction potential

$V_{t} \quad$ Rise velocity

VOF Volume of fluid

W Width of the Domain

\section{GREEK LETTERS}

$\Omega \quad$ Collision operator

$\varsigma \quad$ Circularity

$\kappa \quad$ Component index

$\delta \quad$ Delta Dirac function

$\mu_{H} \quad$ Dynamic viscosity of the liquid phase

$\mu_{L} \quad$ Dynamic viscosity of the gas phase

$\psi \quad$ Effective density

$\rho_{L} \quad$ Gas density

$v_{H} \quad$ Kinematic viscosity of the liquid phase

$v_{L} \quad$ Kinematic viscosity of the gas phase

$\varphi \quad$ Level set function

$\rho_{H} \quad$ Liquid density

$\sigma \quad$ Surface tension

$\omega_{a} \quad$ Weighting factor in the $a$ direction 


\section{INTRODUCTION}

\subsection{Problem Statement and Motivation}

Multiphase systems, and more importantly, gas-liquid mixtures, are of great interest in many industrial (e.g., pulsed-air mixing, involving injection of inert gas in a liquid-filled tank) and natural (e.g., methane generation in peats) processes. Gas bubbles and/or liquid droplets are typical forms of such two phase flows. The investigation of such multicomponent and multiphase flows within the complicated geometries of porous media and in bubbleinduced sediment suspension is crucial to better understand processes found in both aforementioned environments.

The study of the fundamentals of bubble rise is crucial in a variety of practical applications ranging from the rise of steam in boiler tubes to gas bubbles in oil wells [1]. In addition, significant amount of methane $\left(\mathrm{CH}_{4}\right)$, a potent greenhouse gas is generated from peatland ecosystems. Generation of $\mathrm{CH}_{4}$ leads to bubble formation, which can rise through porous media and reach the atmosphere in a process termed ebullition. The nature of ebullition events (in terms of timing and release volumes) of this strong greenhouse gas are not well-known [2]. The application of simulation tools can improve the understanding of the dynamics of this gas in peat soils. 
Although considerable experimental effort has been made to investigate gas generation such as methane fluxes from peatland ecosystems, only a few simulations [3] have been devoted to the analysis of such observations to support existing experimental and semi-empirical findings [4].

In the nuclear industry and specifically in nuclear waste management, pulsed-air mixing, involving injection of discrete pulses of compressed air or inert gas, produces large bubbles that can be used for high-level waste retrieval and processing. As a result of nuclear weapon production, millions of gallons of high-level radioactive waste (HLW) were generated and stored in underground single shell tanks at U.S. Department of Energy (DOE) sites in Hanford [5]. Unfortunately, these single tanks leak and long-lived radioactive materials can contaminate the environment (especially surface and ground water). To prevent such release, pulsed air mixing is being used to mix the waste to avoid pipe clogging during the transfer to newer double shell tanks. By introducing bubbles at the bottom of the tank, large-scale vortices are induced, mobilizing the solids at the bottom of the tank, and making the tank's environment more homogeneous [6]. One of the issues with reliance on experimental studies is time constraints, which make simulation methods a valuable tool.

Because it is easy to parallelize, and due to its simplicity and ability to simulate complex geometries and multiphase flows, the Lattice Boltzmann 
method (LBM) has been proved to be a useful technique in the computational fluid dynamics (CFD) community and has been widely used through the course of the years [ [7], [8], [9], [10], [11] ].

The present study focuses on developing a new approach for rising bubble simulation using the SC-type multicomponent multiphase LBM. This approach, in which an effective buoyant force is applied to the gas component, faithfully captures observed rising bubble dynamics over a broad range of conditions. This method is validated through comparison with wellestablished observations, correlations, and simulations.

\subsection{Outline}

In Chapter 2, the results of previous experimental and simulation efforts on rising bubbles dynamics are summarized. A brief description of the Level Set Method (LSM) used in the traditional Computational Fluid Dynamics (CFD) approach to this problem is also given. Chapter 3 describes the theoretical aspects of the Lattice Boltzmann Method (LBM) approach, emphasizing multicomponent and multiphase systems. The methodology along with the validation tests and simulation results are presented in Chapter 4. The conclusions and recommendations for future work are considered in Chapter 5. 


\section{LITERATURE REVIEW}

Before the emergences of the Lattice Boltzmann approach, conventional computational fluid dynamics (CFD) approaches that solve the Navier-Stokes $(\mathrm{N}-\mathrm{S})$ equations were extensively used to simulate multiphase flows involving bubbles dynamics. Some of these CFD methods include the level set (LSM), the volume of fluid (VOF), and the front tracking method. This chapter presents a literature review of both traditional CFD and LBM approaches for simulating multiphase flows, and more specifically, rising bubble dynamics. A literature review on experimental results involving bubble flow dynamics as well as a brief description of the level set method is presented. Common boundary conditions used in LBM scheme are described and discussed.

\subsection{Experimental Studies and Correlations}

Bubble rise behavior has been extensively investigated. A rising bubble can be characterized in terms of its rise velocity and shape deformation. Several methods have been presented to accurately describe bubble shape deformation and rising velocity. The behavior of a rising bubble in an infinite fluid medium under the influence of gravitational forces can be grouped into three main regimes (spherical, ellipsoidal, and spherical/ellipsoidal cap) based on three dimensionless parameters, namely, the Eötvös number $(E o)$, Morton number $(M)$, and Reynolds number $(R e)$. These are defined as follows: 
- Eötvös number gives the ratio between the body force (gravitational force) and the surface tension force:

$$
E o=\frac{g \Delta \rho d_{o}^{2}}{\sigma}
$$

- Morton number mainly describes the properties of the continuous surrounding fluid such as density and viscosity:

$$
M=\frac{g \Delta \rho \rho_{L}^{2} v_{L}^{4}}{\sigma^{3}}
$$

- Reynolds number is defined as the ratio between the inertial forces and the viscous forces:

$$
R e=\frac{V_{t} d_{o}}{v_{L}}
$$

where $d_{o}$ is the bubble diameter, $\Delta \rho$ is the density difference between the components, $\rho_{L}$ is the liquid density, $g$ is the gravitational acceleration, $\sigma$ is the surface tension, $v_{L}$ is the kinematic viscosity of the liquid, and $V_{t}$ is the terminal velocity of the bubble.

Clift et al. [12] reported a vast body of literature dealing with the fluid dynamics and heat and mass transfer of bubbles and drops. They proposed a graphical correlation of bubble shape and terminal velocity for a broad range of Morton numbers $\left(1.7 \times 10^{-12} \leq M \leq 1.2 \times 10^{3}\right)$. A more comprehensive range of experimental data and a study of correlation of bubble rise velocity and shape deformation were presented by Bhaga and Weber [13] who revised the results 
in Clift et al. [12] by introducing more boundary lines between bubble shapes. Figure 2.1 summarizes the graphical interpretation of their findings. In their experiment, Bhaga and Weber introduced electronically hydrogen bubble tracer into the liquid to visualize the velocity field around the rising bubble, and reported the nature and the geometry of the wake based on the Reynolds number and the dimension of the bubble. Other correlations and models were proposed to compute the terminal velocity of single bubbles rising in pure and contaminated systems. Zudin [14] proposed an analytical solution for shape and rise velocity for large bubbles. The relative terminal rise velocity of a single gas bubble moving into a liquid phase was studied by Bozzano and Dente [15], who introduced a new friction coefficient to evaluate the terminal velocity for various flow regimes and for various bubble sizes. Joseph [16] investigated the work by Davies and Taylor [17] and proposed a formulation for rise velocity of spherical cap bubbles. Tomiyama [18] studied single bubble flows through an inner sub-channel and proposed semi-empirical correlations between shapes and terminal velocities of single rising bubbles. 


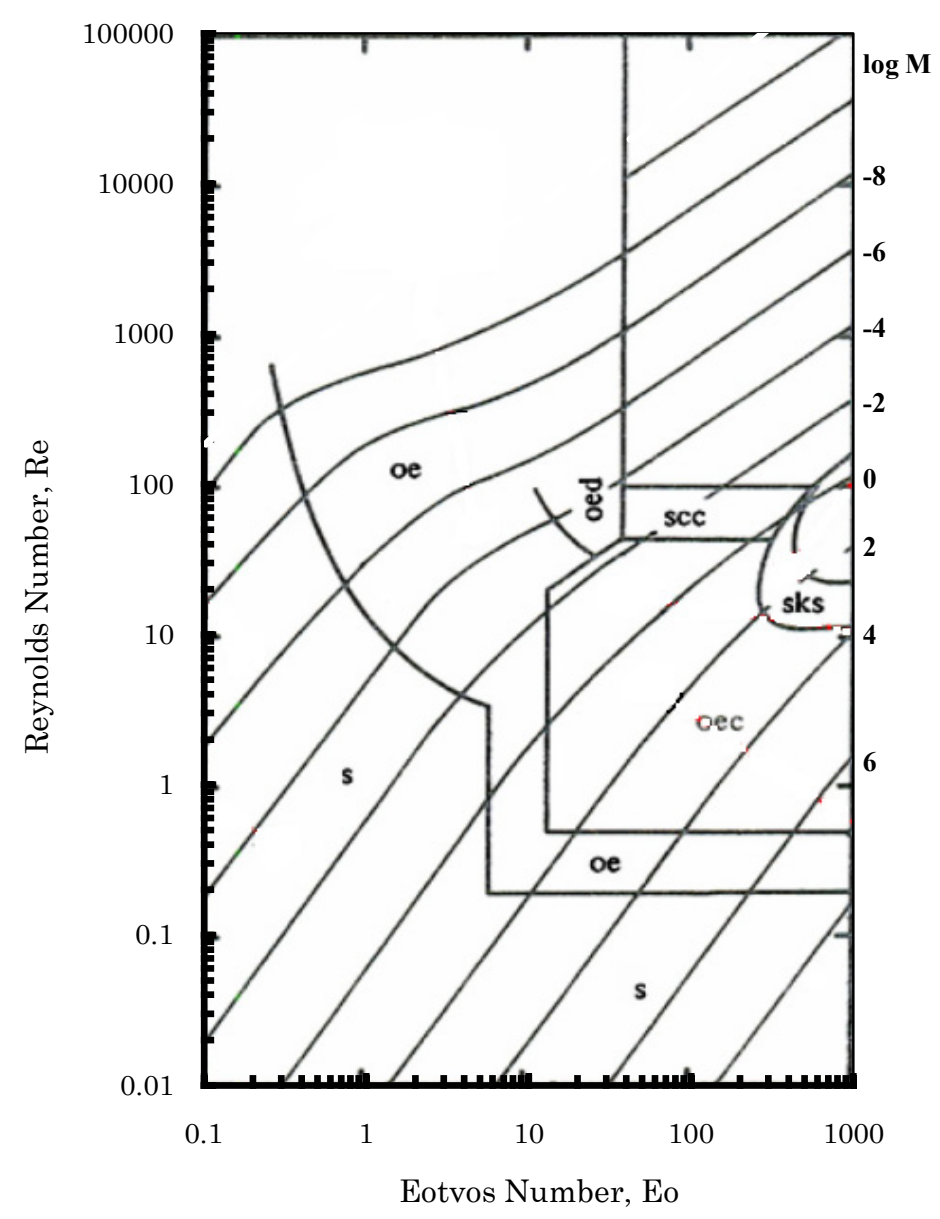

Figure 2.1. Shape regime diagram for bubbles in gravitational motion through liquids. s: spherical; oe: oblate ellipsoid; oed: oblate ellipsoidal (disklike and wobbling); oec: oblate ellipsoidal cap; scc: spherical cap with closed steady wake; sco: spherical cap with open, unsteady wake; sks: skirted with smooth, steady skirt; skw: skirted with wavy, unsteady skirt (adapted from Bhaga and Weber [13])

The wall effect on a single rising bubble, where the fluid outside the bubble is not infinite in extent, was experimentally investigated by Clift et al. [12], Davies and Taylor [17], and recently by Krishna [19]. From experimental observations, they proved that the rise velocity of a bubble is significantly reduced when the ratio of the diameter of the bubble to the diameter of the 
confining column increases, and Krishna suggested an empirical relation for rise velocity for spherical cap in a 2-dimension rectangular column. This wall effect will be investigated in Chapter 4 using Lattice Boltzmann simulations.

\subsection{Multiphase Flow Simulation Using Computational Fluid}

\section{Dynamics}

Although methods such as the level set (LSM), the volume of fluid (VOF), and the front tracking method have been used in conventional CFD solvers to model multiphase flows, only LSM, used as the reference method will be briefly described here; further details on the theory of LSM can be found in [20], and [21]. These traditional CFD approaches used not only the conventional Navier-Stokes (NS) equations to describe the dynamics of each phase, but a set of separate equations is also used to track the interface behavior.

In the LSM, the unsteady, incompressible, Newtonian, and multiphase flows are governed by the NS equations

$$
\begin{gathered}
(\nabla \cdot \boldsymbol{u})=0 \\
\frac{\partial(\rho \boldsymbol{u})}{\partial t}+(\boldsymbol{u} \cdot \nabla \boldsymbol{u})=-\nabla p+\rho \boldsymbol{g}+\left(\nabla \cdot \mu\left[(\nabla \boldsymbol{u})+(\nabla \boldsymbol{u})^{T}\right]\right)+\boldsymbol{F}_{\sigma}
\end{gathered}
$$

In Eq. (2.4), $p$ is the pressure field, $\rho g$ is the gravitational force, $\mu$ is the dynamic viscosity, $\rho$ is the density, and the surface tension force is denoted by $\boldsymbol{F}_{\sigma}$. 
In this approach, the interface is represented as the zero level set of a function with smooth gradient. In high density-ratio multiphase flows, the advection of mass (steep gradient) is replaced with the advection of the smooth level set function. The equation governing the motion of the level set function $\varphi$ is given by:

$$
\frac{\partial \varphi}{\partial t}+\boldsymbol{u} \cdot \nabla \varphi=0
$$

The interface is advected by the fluid velocity $\boldsymbol{u}$ as indicated in Eq. (2.5)

The typical signed distance function $\varphi$ is used to represent the interface between liquid and gas. By tracing an isocontour of the level set function, this application mode finds the interface between the two fluids; that is $\varphi>0$ if in gas region, $\varphi<0$ if in liquid region, $\varphi=0$ if on the interface, and the magnitude of $\varphi$ equals the distance to the interface [21].

For high density and viscosity ratio multiphase simulations, the viscosity $\mu$ and density $\rho$ in Eq. (2.1) are discontinuous across the interface. In such a case, a continuous version of the Heaviside function can be used to smooth out the discontinuity:

$$
\begin{aligned}
& \rho(\varphi)=\left(\rho_{L}-\rho_{G}\right) H(\varphi)+\rho_{G} \\
& \mu(\varphi)=\left(\mu_{L}-\mu_{G}\right) H(\varphi)+\mu_{G}
\end{aligned}
$$

where the Heaviside function $H(\varphi)$ is defined as [21]: 


$$
H(\varphi)=\left\{\begin{array}{cc}
0 & \varphi<-\epsilon \\
\frac{1}{2}+\frac{\varphi}{2 \epsilon}+\frac{1}{2 \pi} \tan \left(\frac{\pi \varphi}{\epsilon}\right) & |\varphi| \leq \epsilon \\
1 & \varphi>\epsilon
\end{array}\right.
$$

where $\epsilon$ corresponds to half the thickness of the interface.

The surface tension force in Eq. (2.4) is evaluated as:

$$
\boldsymbol{F}_{\sigma}=\sigma \eta \boldsymbol{n} \delta(\varphi)
$$

where $\sigma$ is the surface tension, $\eta=\nabla \cdot \frac{\nabla \varphi}{|\nabla \varphi|}$ is the mean curvature, $\boldsymbol{n}=\frac{\nabla \varphi}{|\nabla \varphi|}$ is the normal vector to the interface, and $\delta(\varphi)$ is the delta function defined as

$$
\delta(\varphi)= \begin{cases}\frac{1}{2 \epsilon}\left(1+\cos \left(\frac{\varphi}{\epsilon} \pi\right)\right), & |\varphi| \leq 0 \\ 0, & |\varphi|>0\end{cases}
$$

Results for rising bubble simulations using such conventional CFD methods are available, and only will be briefly summarized here. In 1996, Volkov [22] analyzed the interaction of liquid with a chain of identical gas bubbles by solving the N-S equation. He proved that the maximum flow velocity on the surface of identical bubbles is nearly the same. Olsson and Kreiss [20] presented a conservative method of level set type for moving interfaces in divergence-free velocity fields and applied this approach to single rising bubbles. The wake velocity and the bubble shapes of single and a chain of bubbles were studied by Miyahara et al. [23] for various Tadaki numbers $\left(\operatorname{Re} \times M^{0.23}\right)$. Hoffmann et al. [24] investigated bubbles rising at intermediate Reynolds and large Weber numbers using finite element simulations. Delnoij [25] used the VOF approach to study the time-dependent behavior of 
multiple, "large" gas bubbles rising in an initially quiescent liquid. Koebe [26] investigated gas bubble dynamics in a water-glycerol mixture for small Reynolds numbers, with high density ratio, and using the VOF. Numerical simulations of gas bubble behavior using three-dimensional VOF and front tracking methods, for large density and viscosity ratio were presented by Annaland et al. [27].

\subsection{Multiphase Lattice Boltzmann Method}

The Lattice Boltzmann Method (LBM) has emerged as an alternative and powerful tool for simulating complex fluids in complicated geometry. The four main Lattice Boltzmann-based approaches for analyzing two-phases flows, and based on the Bhatnagar-Gross-Krook (BGK) collision model, were developed by Gunstensen et al. [28], (color-fluid), Shan and Chen [29], pseudo-potential), Swift et al. [30] (free-energy), and He et al. [31] (meanfield theory). A detailed description of all these methods is beyond the scope of this thesis and only the Shan and Chen (SC) approach will be described in the next chapter.

The two-phase model proposed by Gunstensen is based on the original multiphase lattice-gas (LG) model developed by Rothman and Keller [32] and known as the color-fluid model. The LG approach is associated with unphysical effects such as lack of Galilean invariance, and inability to model 
fluids with different densities and viscosities. However, Grunau et al. [33] improved this model to allow for density and viscosity variation.

To model the interaction between the components (two in my study), Shan and Chen proposed an interparticle potential model in which the collision operator is modified by adding an interaction forcing term to the velocity field. The interaction potential is defined based on the local density. A suitable choice of this potential guarantees phase separation, controls the equation of state (EOS), and introduces surface tension effects. Apart from its shortcomings because of lack of interface sharpness, high viscosity, and spurious current, the Shan and Chen model has been proved to be exceptionally versatile [ [11], [34], [7] ] and some improvements have been made to model non-ideal fluids. For instance, to obtain a suitable equation of state (EOS), Yuan and Schaefer [35] modified the SC interparticle potential model. Chibbaro et al. [36] quantitatively studied the second neighbor repulsive interaction potential to control the surface tension independently of the EOS. More recently, Yu and Fan [37] proposed a multirelaxation time (MRT) SC-based LBM for two phase flow. Their MRT model was able to reduce the lowest viscosity in the single relaxation time (SRT) by an order of magnitude, and significantly decreases the spurious current at the gas-liquid interface. 
The so-called free-energy based model [30] describes non-equilibrium dynamics such as Cahn-Hilliard's approach, and incorporates it into the LB model using the free-energy function. Unlike the SC model, the momentum is locally conserved in the free-energy model. This original model lacked Galilean invariance, but that was solved by Pooley and Furtado [38], who introduced an additional term to the equilibrium distribution function. A few modifications have also been introduced by Inamuro et al. [39] and Zheng et al. [9] to achieve high density ratio simulations. Inamuro proposed the pressure projection method, whereas Zheng used the Cahn-Hilliard equation to describe the evolution of the interface.

Proposed by $\mathrm{He}$ et al. [31], the mean-field theory treats the interparticle interaction using the mean-field approximation. The mean-field theory model uses two set of particle distribution functions (pdfs) to simulate the hydrodynamic properties. The first set of pdf describes pressure and velocity fields, whereas the second set tracks the dynamics of the interface.

More recently, computational studies on the evaluation of different shape regimes and rising velocity were developed using Lattice Boltzmann Method. Sankaranarayanan et al. [ [11], [40] ] have explored the SC-LBM and developed an implicit LBM-BGK scheme to probe the flow characteristics of bubble dynamics with $E o<5$. This approach achieves small Morton numbers $\left(M \sim 10^{-10}\right)$ and Eötvös numbers of order unity. Takada et al. [41] presented a 
comparison study of bubble flow between LBM based on the free energy approach and the volume of fluid method. In more extended results, they simulated bubble coalescence processes. Frank et al. [42] used the free energy model for a single bubble rising for Reynolds numbers less than 1.8. Inamuro et al. [39] proposed a heuristic LBM approach in which two particle velocity distribution functions are used to simulate two-phase immiscible fluids with large density ratio. Kurtoglu and Lin [43] assessed the applicability of LBM based on phase-field to single bubble dynamics for density ratio of 2.45 and Eo $<100$. Zheng et al. [9] proposed a free energy approach for multiphase flow with large density ratio, which can be beyond 1000. Gupta and Kumar [7] studied the applicability of SC model to assess the physics of bubble motion and shape evolution for $0.23 \leq \mathrm{Eo} \leq 32$ and $\mathrm{Re}<40$. More recently, Thommes et al. [44] proposed a scheme built on the LBM coupled with the level set method to compute the evolution of the interface. Amaya-Bower and Lee [45] presented a study on dynamics of a single bubble rising for moderate Reynolds numbers using the LBM based on the Cahn-Hilliard diffuse interface approach. Huang et al. [46] applied the 3D free-energy-based LB model to study an air bubble rising in viscous fluid and to test the model performance on large density-ratio and low Reynolds number two-phase flows. Fakhari and Rahimian [47] combined the Multi-relaxation time Lattice Boltzmann method and the axisymmetric LBM model to simulate the buoyancy-driven motion of a rising bubble for low viscosity and very low 
Morton number. Chao et al. [48] assessed some issues of He-Chen-Zhang (HCZ) Lattice Boltzmann model for immiscible multiphase flows with large density ratio. They extended the HCZ model with a filter technique and mass correction procedure.

\subsection{The Boundary Conditions in LBM}

Various classes of boundary conditions have been proposed by many investigators in Lattice Boltzmann Method. Known as the wall boundary conditions, the bounceback (BB) condition was originally taken from the Lattice Gas method proposed by Wolfram in 1986 [49]. Due to its simplicity, the $\mathrm{BB}$ condition has been widely used and is still the most preferred no-slip boundary condition for simulating fluids in complex geometries such as those found in porous media. In the BB scheme, the momentum from the incoming particle that streams and hits the wall is bounced back along the direction opposite where it came from. Several variants of BB were proposed. Inamuro et al. [50] proposed a non-slip boundary condition where the unknown distribution functions (inward-pointing links) at the wall are assumed to be a Maxwell distribution with a counter slip-velocity which is evaluated in such a way that the fluid velocity at the wall equals the wall velocity. However, their method is only accurate for non-slip flat boundary and cannot handle corners. Noble et al. [51] have pointed out that the BB boundary conditions give first order accuracy and proposed an alternative by adding a constraint to 
maintain the internal energy constant under steady flow conditions. Ginzbourg and d'Humieres [52] proposed a rigorous theoretical treatment of the wall boundary with a second order accuracy. However, their approach is not easy to implement and has not been as widely adopted as the BB scheme.

In their method, Maier at al. [53] formulated supplemental rules which employ extrapolation (for density conditions) and mass addition and redistribution (for velocity conditions) for the external links. The implementation of these rules allows solving the number of unknown particle distribution functions at the boundaries which can exceeds the number of equations in 3D lattices. Chen et al. [54] proposed a simple extrapolation scheme considering that the Lattice Boltzmann scheme can be regarded as a special finite difference solution of the kinetic equation. With the idea that for any given fluid flow, there is one additional layer of sites beyond the boundary, they enforced the following condition each time step for the outside nodes before streaming:

$$
f_{i}^{O L}=2 f_{i}^{W}-f_{i}^{I L}
$$

where $f_{i}^{O L}, 2 f_{i}^{W}$, and $f_{i}^{I L}$ represent the distribution functions on the outside layer, the wall layer, and the first layer inside the fluid, respectively.

A new method for pressure and velocity boundary conditions was proposed by He and Zou [55] using half-way wall BB boundary conditions. In their approach, the BB boundary condition was extended for the non-equilibrium 
part of the particle distribution function to determine the inward density distribution functions. Their method was tested for 3-D Poiseuille flow with pressure and velocity inlet/outlet conditions, and found to be of second order accuracy. Mei et al. [56] proposed a scheme to treat curved boundaries. Their method was tested against 2-D benchmark problems such as pressure-driven channel flow and lid-driven, square cavity flow, and was found to be of second order accuracy. Later on, they generalized their method for 3D geometry with the same accuracy and stabilities [57]. 


\section{LATTICE BOLTZMANN FORMULATION}

The Lattice Boltzmann methods (LBMs) have developed into a promising alternative to traditional Computational Fluid Dynamics (CFD) for simulating single and multicomponent multiphase fluid flows. While the level-set method (LSM) coupled with the continuum Navier-Stokes equation solvers has been successfully used for two-phase flow systems [20], LBM has been proved to have various advantages over conventional CFD methods. Such advantages, including implementation of boundary conditions, high performance and efficiency on parallel computing platforms, simulation of complex geometries, and a "natural" ability to simulate the interface between multiple phases have led the LBM to become a promising tool in the CFD community.

\subsection{Single Component Lattice Boltzmann Method}

Historically, the LBM derives from the lattice gas cellular automata (LGCA) model proposed in 1986 by Frisch, Hasslacher, and Pomeau [58]. The LGCA used a Boolean operator to characterize the presence of particle at the lattice site. Known as a six-velocity or FHP model, this LGCA lacks Galilean invariance, presents anomalous pressure dependent velocity, and instabilities and complexity occur at low viscosity [59]. Later on, McNamara and Zanetti [60] helped to circumvent some of these shortcomings in the LGCA, which later evolved to the well-established single relaxation time LBM. 
The Boltzmann equation, which is continuous in the space and velocity variables, constitutes the core of the Lattice Boltzmann models. In the LBM schemes, one solves the kinetic equation, and a discrete set of particle distribution functions $f$, in both time and space are used to describe the fluid dynamics. Unlike conventional CFD approaches, which obtain the macroscopic variables (density and momentum) by solving the Navier-Stokes equations, LBM obtains macroscopic variables by calculating the hydrodynamics moments of the distribution function.

Introduced by Bhatnagar-Gross-Krook (BGK) [61], the single relaxation time collision model has been widely used and adopted in the LBM community. The kinetic equation is

$$
\frac{\partial f}{\partial t}=\boldsymbol{e} \cdot \boldsymbol{\nabla} f=\frac{1}{\tau}\left(f-f^{e q}\right)
$$

where $f^{e q}$ is the equilibrium distribution function (the Maxwell-Boltzmann distribution function), $\boldsymbol{e}$ is a particle velocity vector and $\tau$ is the relaxation time.

In the 2 dimensional, nine velocity, square lattice model known as D2Q9, Figure 3.1, the fluid dynamics is described by the following discrete velocity BGK equation:

$$
\frac{\partial f_{a}}{\partial t}=\boldsymbol{e}_{\boldsymbol{a}} \cdot \boldsymbol{\nabla} f_{a}=\frac{1}{\tau}\left(f_{a}-f_{a}^{e q}\right), \quad 0 \leq a \leq 8
$$


where $f=f_{a}(\boldsymbol{r}, t) \equiv f\left(\boldsymbol{r}, \boldsymbol{e}_{a}, t\right)$ is the distribution function associated with the ath discrete velocity $\boldsymbol{e}_{a}$, at position $\boldsymbol{r}$ and time $t$. The relaxation time $\tau$ is related to the kinematic viscosity as:

$$
v=c_{s}^{2}(\tau-0.5 \delta t)
$$

The discrete possible velocities, $\boldsymbol{e}_{a}$ s are defined as:

$$
\boldsymbol{e}_{a}=\left\{\begin{array}{lr}
(0,0) & a=0 \\
(1,0),(0,1),(-1,0),(0,-1) & a=1,2,3,4 \\
(1,1),(-1,1),(-1,-1),(1,-1) & a=5,6,7,8
\end{array}\right.
$$

The equilibrium distribution function for D2Q9 is defined as

$$
f_{a}^{e q}=\rho \omega_{a}\left[1+\frac{\boldsymbol{e}_{a} \cdot \boldsymbol{u}}{c_{s}^{2}}+\frac{\left(\boldsymbol{e}_{a} \cdot \boldsymbol{u}\right)^{2}}{2 c_{s}^{4}}-\frac{\boldsymbol{u}^{2}}{2 c_{s}^{2}}\right]
$$

where $c_{s}=\frac{c}{\sqrt{3}}$ is the speed of sound, $c=\frac{\delta x}{\delta t}=1$ is the lattice constant. The weighting factors, $\omega_{a}$, are defined as follows:

$$
\omega_{a}=\left\{\begin{array}{lr}
4 / 9, & a=0 \\
1 / 9, & a=1,2,3,4 \\
1 / 36, & a=5,6,7,8
\end{array}\right.
$$

Unlike traditional CFD methods, which directly solve for the transport equations of the macroscopic variables (density and momentum), the LBM solves for the particle distribution function (pdf) and the macroscopic variables are obtained as the hydrodynamic moments of this pdf:

$$
\rho=\sum_{a} f_{a}
$$




$$
\rho \boldsymbol{u}=\sum_{a} \boldsymbol{e}_{a} f_{a}
$$

The pressure field is defined by the equation of state of an ideal gas

$$
p=\rho c_{s}^{2}
$$

The complete discretization of the BGK collision model with time step $\delta t$ and space step $\delta \boldsymbol{r}$ leads to the lattice Boltzmann BGK model (LBGK).

$$
f_{a}\left(\boldsymbol{r}+\boldsymbol{e}_{a} \delta t, t+\delta t\right)-f_{a}(\boldsymbol{r}, t)=\Omega_{\mathrm{a}}
$$

where $\Omega_{\mathrm{a}}=\frac{f_{a}^{e q}-f_{a}}{\tau}$ is the collision term.

Streaming and collision of the distribution function constitute the whole conceptual framework of the model [62]. The collision step is completely local and described by:

$$
\widetilde{f_{a}}(\boldsymbol{r}, t)-f_{a}(\boldsymbol{r}, t)=\frac{f_{a}^{e q}(\boldsymbol{r}, t)-f_{a}(\boldsymbol{r}, t)}{\tau}
$$

The streaming describes the propagation of particles from cells into neighboring cells. This streaming step involves no computation and is considered an intermediate state in the model.

$$
f_{a}\left(\boldsymbol{r}+\boldsymbol{e}_{a} \delta t, t+\delta t\right)=\widetilde{f}_{a}(\boldsymbol{r}, t)
$$

where $f_{a}$, and $\widetilde{f}_{a}$ are the pre- and post-collision states of the distribution function, respectively. 


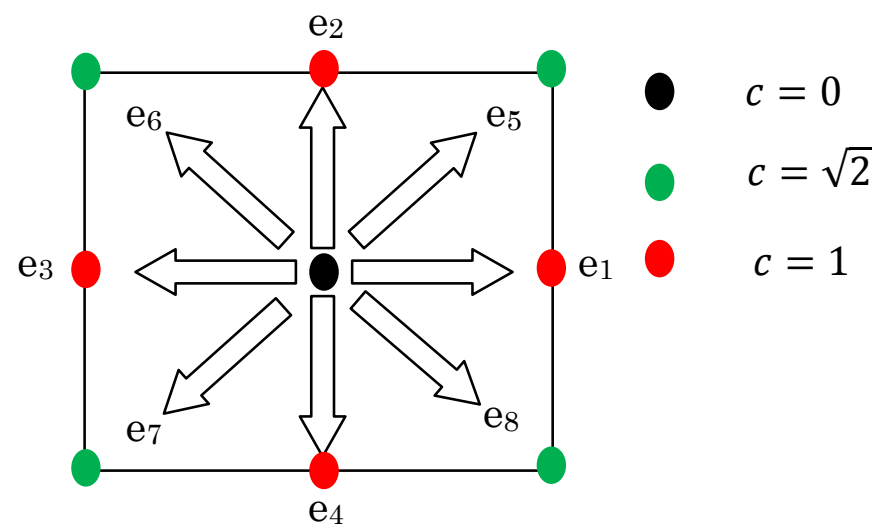

Figure 3.1. D2Q9 lattice model

Although other two-dimensional schemes are in use, the D2Q9 model seems to be the most popular lattice scheme. Mezrhab et al. [63] illustrated the use of the D2Q5 in their recent study in simulating convective flow with MRTLBM. In this lattice model, each lattice site is linked with four neighbors as illustrated in Figure 3.2. In three dimensional schemes, the D3Q15, D3Q19, and D3Q27 lattices are used. The main parameters such as the associated weighting factors can be found in [59].

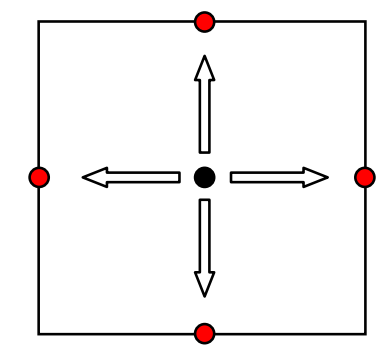

Figure 3.2. D2Q5 lattice model 


\subsection{Multicomponent Multiphase Lattice Boltzmann Method}

For the past two decades, multiphase fluid flow using LBM has been a subject of many studies. Shan and Chen (SC) [29] proposed a multiple phases LBM model by introducing an interparticle potential between fluid components and based on the BGK collision model. Subsequently, Swift et al. [30] introduced the free energy method to correct the unphysical velocity fluctuations at the interface in the SC model. More details on these multicomponent flow models can be found in [ [31], [39], [64]. In this research work, the isothermal SC LBM is used and is described in the next section.

\subsection{Shan and Chen Interparticle Potential Model}

Shan and Chan developed one of the first LBMs for multiphase and multicomponent flows in which one distribution function is introduced for each of the fluid components [29]. Equations presented in Section 3.1 for the single component remain valid for the multicomponent models, except that an index $\kappa$ is introduced to account for each of the component. In this work $\kappa=1,2$ and

$$
f_{a}^{\kappa}\left(\boldsymbol{r}+\boldsymbol{e}_{a} \delta t, t+\delta t\right)-f_{a}^{\kappa}(\boldsymbol{r}, t)=\Omega_{\mathrm{a}}^{\kappa}(\boldsymbol{r}, \mathrm{t})
$$

where $\Omega_{\mathrm{a}}^{\kappa}=\frac{f_{a}^{\kappa, e q}-f_{a}^{\kappa}}{\tau_{\sigma}}$ is the BGK collision term, $\tau_{\kappa}$ is the relaxation parameter related to the kinematic viscosity by

$$
v_{\kappa}=c_{S}^{2}\left(\tau_{\kappa}-0.5 \delta t\right)
$$

The equilibrium distribution function is evaluated as: 


$$
f_{a}^{\kappa, e q}=\rho_{\kappa} \omega_{a}\left(1+3 \boldsymbol{e}_{a} \boldsymbol{u}_{\boldsymbol{\kappa}}^{\boldsymbol{e q}}+\frac{9}{2}\left(\boldsymbol{e}_{a} \boldsymbol{u}_{\boldsymbol{\kappa}}^{\boldsymbol{e q}}\right)^{2}-\frac{3}{2} \boldsymbol{u}_{\boldsymbol{\kappa}}^{\boldsymbol{e q} \boldsymbol{q}^{2}}\right)
$$

In order to incorporate nonlocal interaction among particles, Shan and Chen defined an interaction potential between components $\kappa$ and $\bar{\kappa}$.

$$
V\left(\boldsymbol{r}, \boldsymbol{r}^{\prime}\right)=G_{\kappa \bar{\kappa}}\left(\boldsymbol{r}, \boldsymbol{r}^{\prime}\right) \psi^{\kappa}(\boldsymbol{r}) \psi^{\bar{\kappa}}\left(\boldsymbol{r}^{\prime}\right)
$$

where $\boldsymbol{r}^{\prime}=\boldsymbol{r}+\boldsymbol{e}_{a} \delta t$ is the location of the neighbor sites, $\psi^{\kappa}$ and $\psi^{\bar{\kappa}}$ are commonly taken as the effective density of components $\kappa$ and $\bar{\kappa}$, respectively. $G_{\kappa \bar{\kappa}}$ is the strength of the interaction controlling the surface tension. It assumes the form of a Green's function. The magnitude of $G_{\kappa \bar{\kappa}}$ controls the interaction strength between the components and its sign determines whether the interaction is attractive or repulsive.

For nearest neighbor interactions, $G_{\kappa \bar{\kappa}}$ is defined as:

$$
G_{\kappa \bar{\kappa}}\left(\mathbf{r}, \mathbf{r}^{\prime}\right)=\left\{\begin{array}{cc}
0 & \left|\mathbf{r}-\mathbf{r}^{\prime}\right|>\left|\mathbf{e}_{\mathrm{a}} \delta \mathrm{t}\right| \\
g_{\kappa \bar{\kappa}} & \left|\mathbf{r}-\mathbf{r}^{\prime}\right|=\left|\mathbf{e}_{\mathrm{a}} \delta \mathrm{t}\right|
\end{array}\right.
$$

The interaction strength parameters $G_{11}$ and $G_{22}$ describe the interaction within each individual component. If the first component is assumed to be a non-ideal fluid the equation of state (EOS) is given by

$$
P=\rho R T+G_{11} \frac{R T}{2}[\psi(\rho)]^{2}
$$

The potential $\psi(\rho)$ must be monotonically increasing and bounded to be consistent with isothermal process. Different forms of $\psi(\rho)$ has been adopted 
in the literature $[[\mathbf{2 9}],[\mathbf{6 5}],[\mathbf{6 6}]]$. The original form of the interaction potential introduced by Shan and Chen is widely used and is given as follows

$$
\psi(\rho)=1-\exp (-\rho)
$$

If the second component is an ideal, $\mathrm{G}_{22}=0$ and $\psi(\rho)=\rho$. The interaction between the two components and the immiscibility of the mixture are described by $\mathrm{G}_{12}$.

Given this form of potential, the rate of net momentum change induced at each site due to the interaction is simply given as in [29]:

$$
\frac{d p^{\kappa}(\boldsymbol{r})}{d t}=-\nabla V\left(\boldsymbol{r}, \boldsymbol{r}^{\prime}\right)=-\psi^{\kappa}(\boldsymbol{r}) \sum_{\bar{\kappa}=1}^{S} G_{\kappa \bar{\kappa}} \sum_{a=0}^{b} \psi^{\bar{\kappa}}\left(\boldsymbol{r}+\boldsymbol{e}_{a} \delta t\right) \boldsymbol{e}_{a}
$$

The change in momentum is applied at each lattice site $\boldsymbol{r}$ before the collision. After a collision, the new net momentum for the component $\kappa$ becomes

$$
\boldsymbol{u}_{\kappa}^{e q}=\boldsymbol{u}^{\prime}+\frac{\tau_{\kappa}}{\rho_{\kappa}} \frac{d p^{\kappa}(\boldsymbol{r})}{d t}
$$

where $\rho_{\kappa}=m^{\kappa} \sum_{a} f_{a}^{\kappa}$ and $\boldsymbol{u}_{\kappa}^{e q}$ are the mass density and the averaged velocity of the $\kappa$ th component, respectively. $\boldsymbol{F}^{\kappa}$ is the total force on component $\kappa$. The velocity $\boldsymbol{u}$ is the meaningful composite macroscopic velocity used to analyze the overall fluid flow system. To satisfy the conservation of momentum at each collision when $\boldsymbol{F}^{\kappa}=0, \boldsymbol{u}$ must satisfy the relation [29] 


$$
\boldsymbol{u}^{\prime}=\frac{\sum_{\kappa} \frac{m^{\kappa}}{\tau_{\kappa}} \sum_{a} f_{a}^{\kappa} \boldsymbol{e}_{a}}{\sum_{\kappa} \frac{m^{\kappa}}{\tau_{\kappa}} \sum_{a} f_{a}^{\kappa}}
$$

The density and the macroscopic velocity of the mixture are defined as

$$
\begin{gathered}
\rho=\sum_{\kappa} \rho_{\kappa} \\
u=\sum_{\kappa} \rho_{\kappa} u_{\kappa}+\sum_{\kappa} F_{\kappa}
\end{gathered}
$$

Particle momentum at each site is not conserved because of the interaction potential. However, the total momentum of the system is exactly conserved. The application of the multicomponent SC LBM-type model for rising bubble simulation will be presented in the next chapter. 


\section{SIMULATION OF SINGLE AND MULTIPLE BUBBLES}

Chapter IV presents numerical results of single and multiple bubble simulations. A new effective buoyancy method was developed to capture the dynamics of a bubble in a quiescent liquid. A benchmark case involving simulation of static bubbles for interfacial tension is included to validate the basic multiphase code. A test case for specific Eötvös and Morton numbers was performed and compared with a reference LSM approach as a test of the new method. Interaction and coalescence between multiple bubbles were also investigated and analyzed. A brief description of bubble flow in porous media was also introduced and implementation of source terms for gas generation was performed.

\subsection{Test Case Evaluation of Multicomponent Simulation}

\subsubsection{Laplace Test: Interfacial Tension Evaluation}

The interfacial tension was evaluated based on Laplace's equation given by the following expression

$$
\Delta P=P_{\text {in }}-P_{\text {out }}=\frac{\sigma}{R}
$$

Where $\mathrm{R}$ is the radius of a circular bubble, $\sigma$ is the surface tension, and $\Delta P$ is the pressure difference between the inside and the outside of the bubble. A series of simulations of bubbles of different sizes was performed in a $100 \times 100$ $\mathrm{lu}^{2}$ domain. The domain boundary used periodic boundary conditions. The simulation parameters include the interaction strength $G_{12}=3.5$, which 
reduces the diffusivity to a relatively low value and assures phase separation. The relaxation time, $\tau_{1}=\tau_{2}=1$ for both components. Initially, inside and outside the initial bubble, the density of phase 1 was set to 1 and 10-10, respectively, and for phase 2 it was set to $10^{-10}$ and 1 , respectively. Simulations were run for 50,000 time steps to assure steady state. Figure 4.1 illustrates the density contour and the velocity field at the interface of the two fluid components.

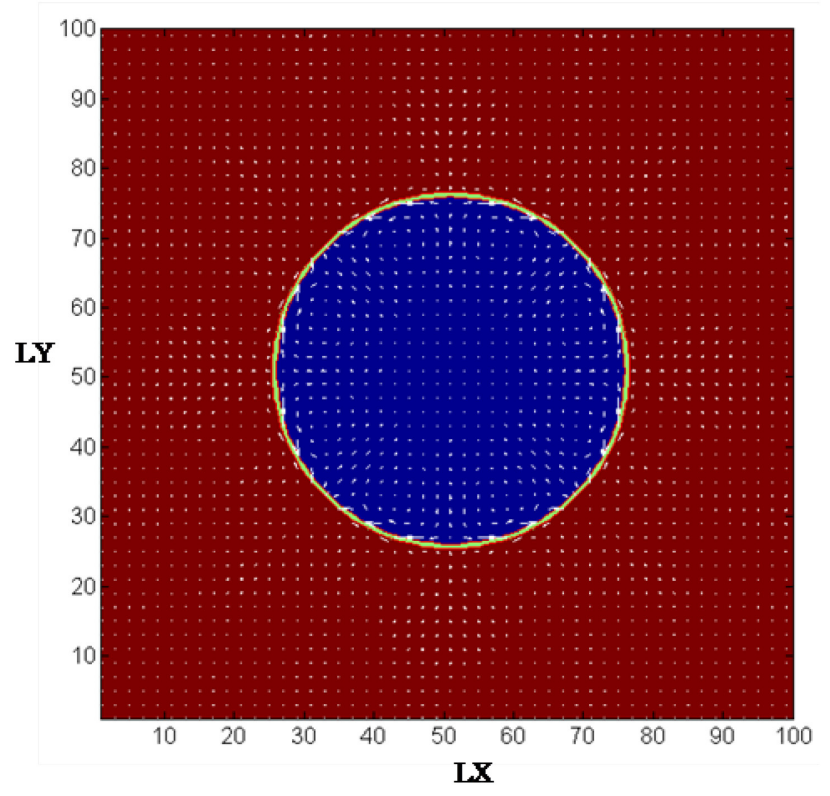

Figure 4.1. Steady density contour and velocity field for a static bubble. The blue bubble of radius $\mathrm{r}=25 \mathrm{lu}$ is surrounded by the red liquid. Domain $100 \times 100 \mathrm{lu}^{2}$. Time $=50,000 \mathrm{ts}, G_{12}=3.5$

The observed velocity field (circulating flow) near the interface of the stationary bubble is known as the spurious velocity (Figure 4.2) and is the results of the non-local conservation of momentum at each lattice site. The 
spurious velocity can be significantly reduced by using a high order discretization of the density gradient [67].

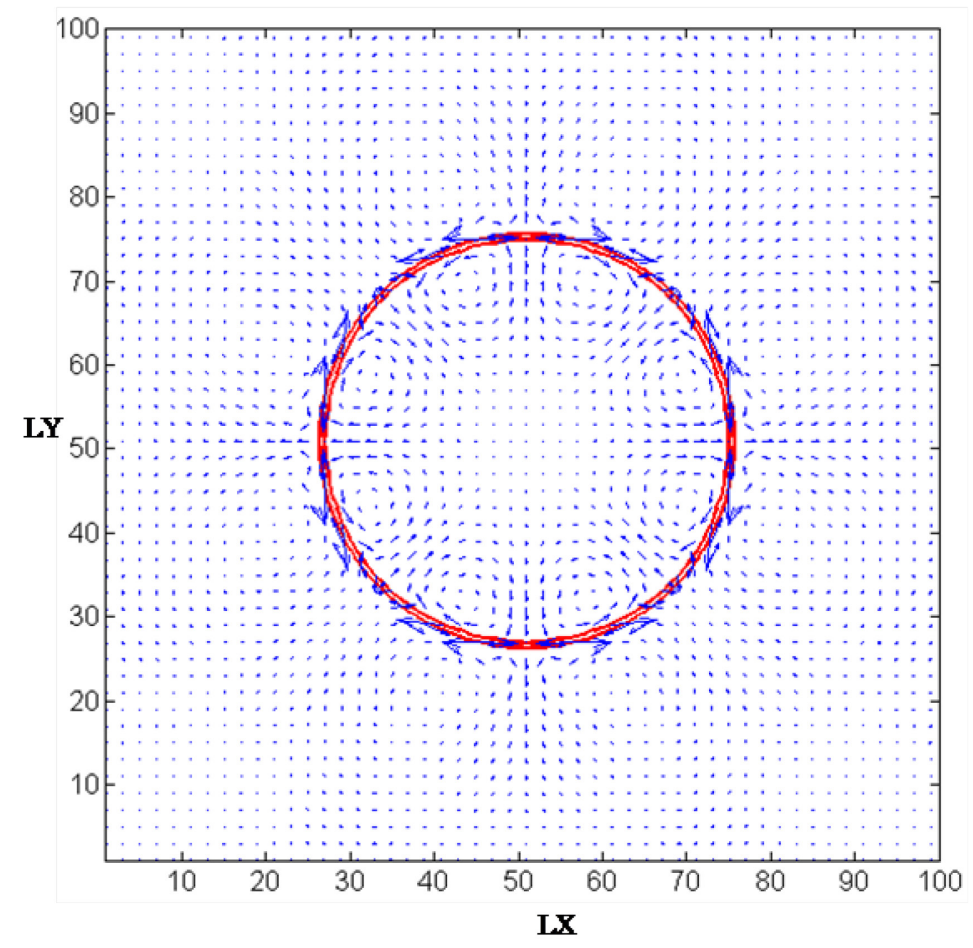

Figure 4.2. Spurious velocity

Figure 4.3 presents the pressure difference between the inside and the outside of a series of bubbles of different sizes as a function of the inverse bubble radius. It can be seen that the Laplace's law is satisfied. The linear relationship between $\Delta P$ and the inverse of the bubble radius confirms a constant value of the surface tension, representing the slope of the line defined by the points on the plot. 




Figure 4.3. Pressure difference between the inside and the outside of a series of bubbles as a function of inverse bubble radius. $G_{\kappa \bar{\kappa}}=3.5, t=50,000$ time steps.

\subsubsection{Case Comparison of Single Rising Bubble}

The rise of a bubble is the result of gravity and the difference in density between the gas and the liquid. In order to consider the buoyant effect associated with density difference between liquid and gas, an effective buoyant force, $F^{e, \kappa}$ was introduced. It is defined by Eq. (4.2):

$$
F^{e, \kappa}=\rho_{\kappa} \times g_{\text {applied }}
$$

In fact, because of the density difference between the phases, the buoyancy is defined as:

$$
B=g_{e f f} \Delta \rho_{e f f}=g_{e f f}\left(\rho_{H}-\rho_{L}\right)
$$




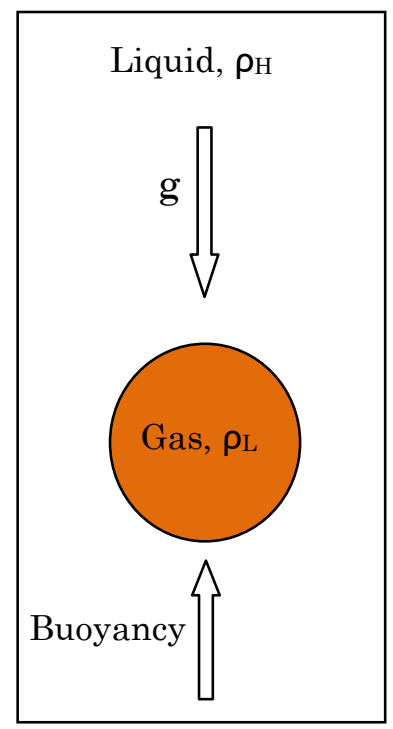

Figure 4.4. Representation of the buoyancy force versus the weight force. $\rho_{H}$ is the heavier liquid density, and $\rho_{L}$ the lighter gas density.

Equating Eqs. (4.2) and (4.3) yields to:

$$
g_{\text {applied }}=\frac{g_{e f f}\left(\rho_{H}-\rho_{L}\right)}{\rho_{\kappa}}
$$

where $\rho_{H}$ is the heavier liquid density, $\rho_{L}$ is the lighter gas density, and $\rho_{\kappa=L}$ is the density of the gas phase as actually used in the simulation. The $g_{\text {applied }}$ represents the gravitational acceleration actually used in the simulation and applied to component $\kappa$ only. Thus $g_{\text {eff }} \Delta \rho_{\text {eff }}$ are the effective physical gravitational acceleration and density difference quantities, while the $\rho_{\kappa=L}$ and $g_{\text {applied }}$ are simply simulation surrogates that yield the same buoyancy effect. This approach of placing the buoyant effects into a body force greatly expands the range of effective density difference between bubble and liquid 
that can be readily simulated with the $\mathrm{SC}$ model. The $g_{e f f} \Delta \rho_{e f f}$, which controls the buoyancy, appears in the dimensionless Eötvös, and Morton numbers defined in Chapter two. Test cases using this approach are presented in the next section and comparisons are made with the wellestablished level set method. One drawback of this new approach, in which only the bubble experiences a body force, is that there is no hydrostatic pressure gradient in the fluid outside the bubble; a slightly more elaborate scheme should readily permit that to be incorporated.

\section{Validation of the reference level set method}

To verify my LBM results, the LSM was used in COMSOL Multiphysics [68] as the reference method. A benchmark case for single bubble dynamics was selected to investigate the multiphase capabilities of the LSM, which will later be used to validate the LBM simulations. A test case comparison between the level set methods and the finite element method code (TP2D) developed by Hysing et al. [ [69], [70]] was performed. The parameters of the LSM and TP2D simulations are based on non-dimensional units. The benchmark quantities such as circularity and rise velocity were used to monitor convergence of the two methods. The constant velocity at which the drag, and the buoyancy forces are balanced is called the terminal velocity. The circularity and the rise velocity are defined as: 


$$
\begin{gathered}
\varsigma=\frac{P_{a}}{P_{b}}=\frac{\text { perimeter } \text { of area equivalent circle }}{\text { perimeter of bubble }} \\
V_{t}=\frac{\int \boldsymbol{u}(r, t) d \Omega}{\int d \Omega}
\end{gathered}
$$

where $\Omega$ is the region of the domain occupied by the bubble. A variant is to use the velocity at the centroid of the bubble. The non-dimensional Eötvös and Morton numbers, the density ratio $\frac{\rho_{H}}{\rho_{L}}=10$, and the viscosity ratio $\frac{\mu_{H}}{\mu_{L}}=10$ were used in the test case. Figure 4.5 shows the shape at the final time $\mathrm{T}=4.2$ computed with COMSOL and TP2D for $E o=9$ and $M=6 \times 10^{-4}$. The circularity results obtained by both methods are similar, indicating a difference not greater than $0.7 \%$. The rise velocity is shown in Figure 4.6 as a function of time. As shown, the bubble velocity reaches a constant value after about time $\mathrm{T}=3$, indicating that balance between the buoyancy and the drag force has been reached.

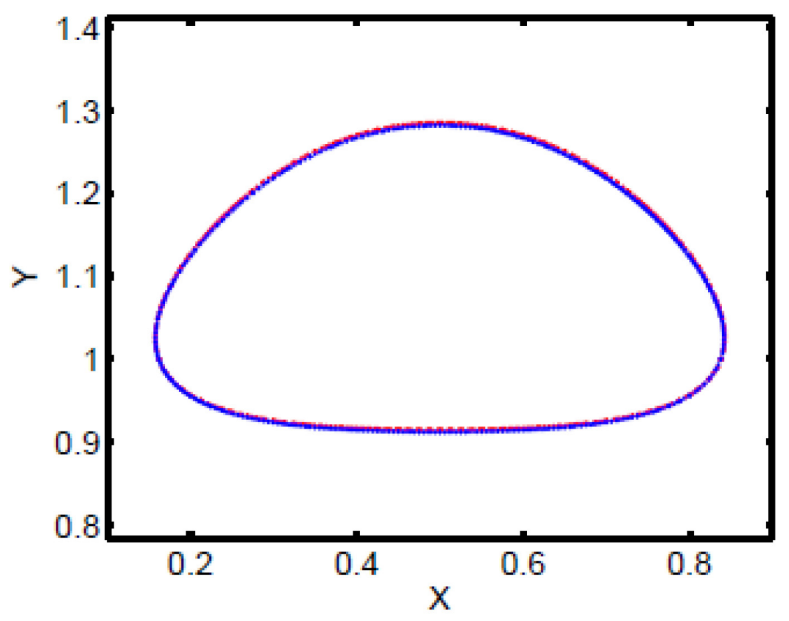

Figure 4.5. Bubble shape at time $\mathrm{T}=4.2$ obtained with the reference method (COMSOL, blue) and the TP2D (red) for Eo $=9$ and $\mathrm{M}=6 \times 10^{-4}$ and a grid size of $50 \times 100$. 
The results agree within $8 \%$ error for the $50 \times 100$ grid level, and the discrepancy could be the result of re-initialization parameters, and the conservative and the non-conservative level-set approaches adopted by each of the methods.



Figure 4.6. Rise velocity as function of time obtained with the reference method (COMSOL, blue) and the TP2D (red) for $E o=9$ and $M=6 \times 10^{-4}$. The grid sizes are shown in parenthesis.

\section{LBM versus LSM}

In order to gauge the capabilities of the LBM algorithm, I conducted a 2-D simulation of a single rising bubble to evaluate the performance of my effective buoyant force. Unless otherwise mentioned, a grid size of $2 d_{o} \times 4 d_{o}$ was used ( $d_{o}=56$ is the initial bubble diameter in lattice units). The time 
used in the simulations was non-dimensionalized by the reference time $t_{\text {ref }}=\sqrt{d_{o} / g_{\text {applied }}}$. I imposed wall boundary conditions at the top and bottom sides of the domain, and periodic boundary conditions on the vertical sides. The evaluation was done for $E_{O}=9$ and $M=6 \times 10^{-4}$. Figure 4.7 shows the simulated bubble evolution under gravity using LBM and LSM. The final shape is quite similar for both methods. Quantitative evaluation was determined by the circularity and terminal Reynolds number as indicated on Figure 4.8 and Figure 4.9, respectively. The similarity of the Reynolds number and the circularity obtained by both LBM and LSM suggests that our effective buoyancy approach is reasonable at least through a density ratio of 10. The relative difference is less than $1 \%$ and $\sim 5 \%$ for circularity and Reynolds number, respectively.
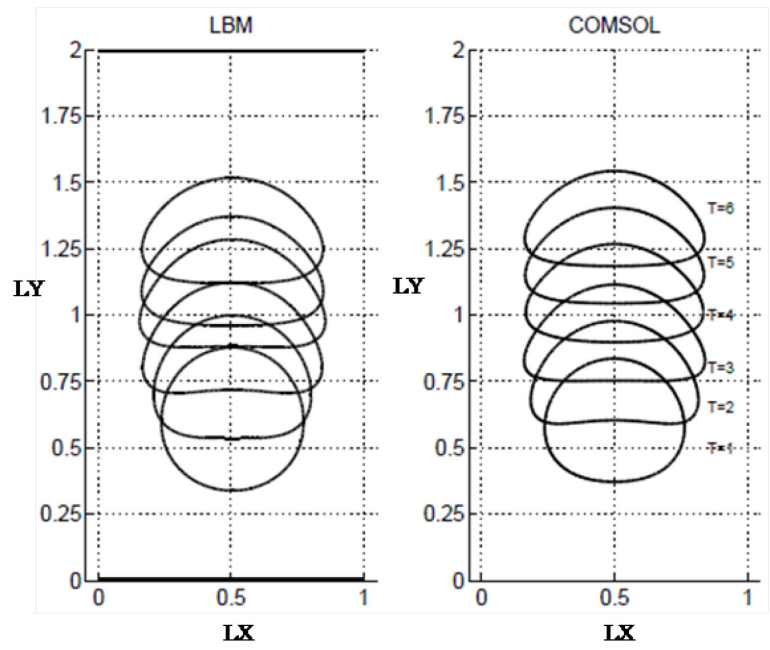

Figure 4.7. Bubble shape evolution at $E o=9$ and $M=6 \times 10^{-4}$, as it forms an oblate ellipsoidal shape, obtained with LBM (left) in a $107 \times 212$ lu domain and LSM (right) in a $40 \times 80$ domain. The time in this plot is dimensionless $\mathrm{T}=$ $\mathrm{t} / \mathrm{t}_{\mathrm{ref}}$. 


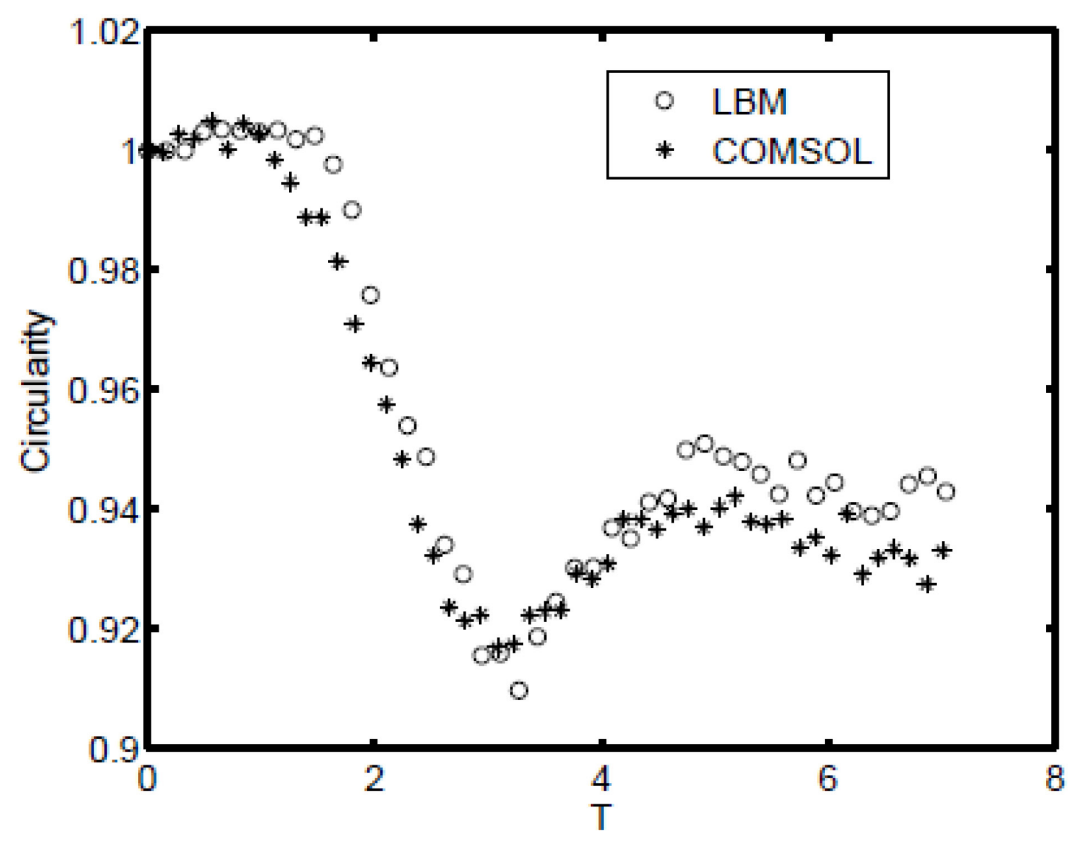

Figure 4.8. Circularity at $E o=9$ and $M=6 \times 10^{-4}$, obtained with LBM and LSM.

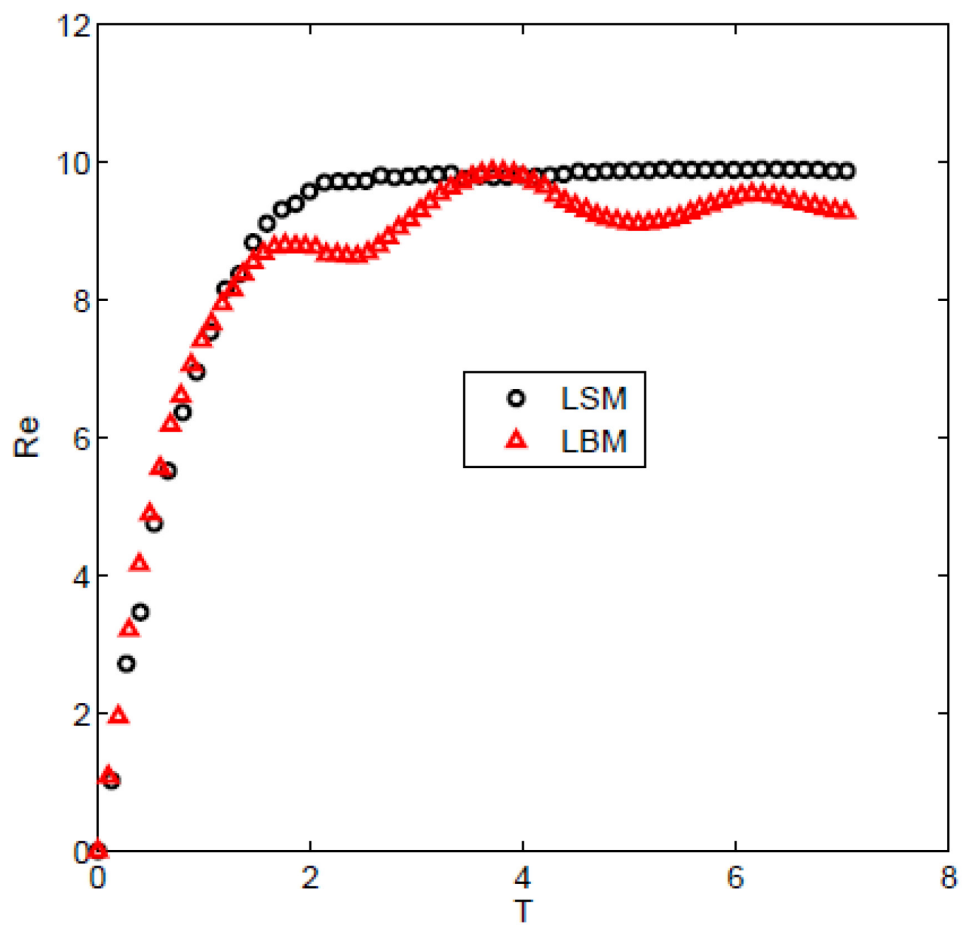

Figure 4.9 : Reynolds number as function of time obtained with LBM and LSM for $E O=9$ and $M=6 \times 10^{-4}$. 
To fully evaluate the proposed buoyancy force, simulations for various flow regimes in comparison with the LSM were carried out and will be discussed in the next section.

\subsection{Numerical Results and Discussion}

Simulations were performed for a broad range of Eötvös and Morton numbers using a completely closed domain. Unless otherwise mentioned, the interfacial tension is $\sigma=0.215 \mathrm{mu} \mathrm{lu} \mathrm{ts}^{-2}$ and 24.5 for LBM and LSM, respectively. Bubble motion was captured for sufficient amount of time to achieve a steady state. The transverse domain size was set to $2 d_{0}$. The vertical size was different in each case, and chosen in such a way that the bubble did not touch the top wall. For $1 \leq E o \leq 10$, the vertical size was $4 d_{o}$, and adjustments were made accordingly for higher Eo values. A bubble of initial diameter $d_{o}=80$ lu and 0.5 was used in LBM and LSM respectively. All the cases were simulated using a kinematic viscosity ratio $v_{H} / v_{L}=1$.

Table 1 gives the parameters used in the current simulations. The magnitude of the applied gravity ("effective buoyancy force") is calculated from the Eötvös number. The kinematic viscosity ranges from 0.166 to $0.306 \mathrm{lu}^{2} \mathrm{ts}^{-1}$ in LBM and $4.5 \times 10^{-3}$ to $8 \times 10^{-3}$ in LSM. Over this range, the Morton number varies from $2.75 \times 10^{-6}$ to $2.73 \times 10^{-3}$. 


\begin{tabular}{llll}
\hline Case & Eo & M & gapplied $\left(\mathbf{l u ~ t s} \mathbf{~}^{-2}\right)$ \\
\hline \hline $1 \mathrm{a}$ & 1 & $2.75 \times 10^{-6}$ & $3.36 \times 10^{-5}$ \\
$1 \mathrm{~b}$ & 1 & $1 \times 10^{-5}$ & $3.36 \times 10^{-5}$ \\
$1 \mathrm{c}$ & 1 & $2.73 \times 10^{-5}$ & $3.36 \times 10^{-5}$ \\
\hline $2 \mathrm{a}$ & 5 & $1.3 \times 10^{-5}$ & $1.68 \times 10^{-4}$ \\
$2 \mathrm{~b}$ & 5 & $5 \times 10^{-5}$ & $1.68 \times 10^{-4}$ \\
$2 \mathrm{c}$ & 5 & $1.37 \times 10^{-4}$ & $1.68 \times 10^{-4}$ \\
\hline $3 \mathrm{a}$ & 10 & $2.75 \times 10^{-5}$ & $3.36 \times 10^{-4}$ \\
$3 \mathrm{~b}$ & 10 & $1 \times 10^{-4}$ & $3.36 \times 10^{-4}$ \\
$3 \mathrm{c}$ & 10 & $2.73 \times 10^{-4}$ & $3.36 \times 10^{-4}$ \\
\hline $4 \mathrm{a}$ & 30 & $7.76 \times 10^{-5}$ & $1.0 \times 10^{-3}$ \\
$4 \mathrm{~b}$ & 30 & $3 \times 10^{-4}$ & $1.0 \times 10^{-3}$ \\
$4 \mathrm{c}$ & 30 & $8.14 \times 10^{-4}$ & $1.0 \times 10^{-3}$ \\
\hline $5 \mathrm{a}$ & 75 & $2 \times 10^{-4}$ & $2.52 \times 10^{-3}$ \\
$5 \mathrm{~b}$ & 75 & $7.46 \times 10^{-4}$ & $2.52 \times 10^{-3}$ \\
$5 \mathrm{c}$ & 75 & $2 \times 10^{-3}$ & $2.52 \times 10^{-3}$ \\
\hline $6 \mathrm{a}$ & 100 & $1.0 \times 10^{-3}$ & $3.36 \times 10^{-3}$ \\
$6 \mathrm{~b}$ & 100 & $2.73 \times 10^{-3}$ & $3.36 \times 10^{-3}$ \\
\hline
\end{tabular}

Table 1. Parameters used in the Lattice Boltzmann simulations

\subsubsection{Bubble Shape, Terminal Rising Velocity, and Reynolds Number}

Clift et al. [12] provided a bubble chart to estimate the shape of a rising bubble in terms of Eötvös number, Morton number, and Reynolds number. This diagram was later revised by Bhaga and Weber [13] who included additional shape regimes and transition lines. Three main categories of bubble shape have been identified:

\section{- Spherical shape}

This category is characterized by bubbles of spherical shape. In fact, the interfacial forces and/or viscous forces are much more important 
than the inertial forces. The terminal velocity is proportional to the size of the bubble and no internal circulation is observed.

\section{- Ellipsoidal shape}

Both surface tension and viscosity affect this regime. The bubbles are of intermediate size and Eötvös number ranges from 0.3 to 40 . Characterization of ellipsoidal shape is particularly difficult since bubbles in this regime tend to oscillate and wobble.

\section{- Spherical cap shape}

This regime is more affected by inertia forces, and characterized by Eo $>40$. The rise velocity is described by the equation proposed by Davies and Taylor [17]. In this regime, large spherical caps may trail thin satellites bubbles known as skirts.

Figure 4.10 shows the blue bubble surrounded by the red liquid as the bubble deforms when rising.

Figure 4.11 shows the bubble shape deformations as calculated with my method, for cases 1a, 2b, and 3b. In the regime of low Eötvös numbers ( $E o \leq$ 1), the bubbles remain spherical while rising in the liquid. With an increase in Eötvös number $(2 \leq E o \leq 30)$, bubbles of oblate ellipsoidal shapes are observed, with the bubble bottom becoming flat or slightly dimpled. 


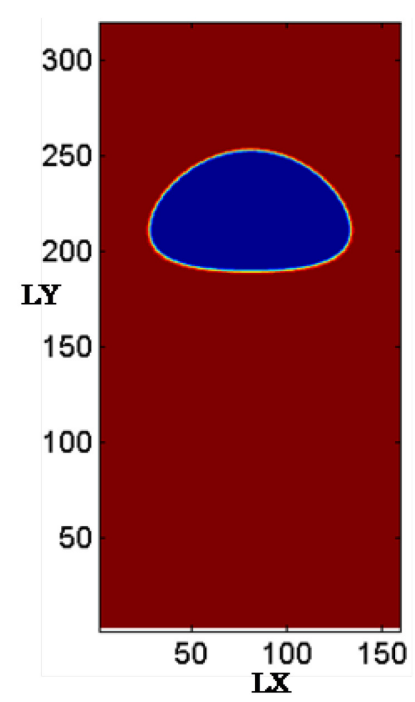

Figure 4.10. Bubble shape for $E o=5$ and $M=1.3 \times 10^{-5}$. The red liquid surrounds the blue bubble.

These flow regimes are mainly dominated by surface tension and viscous forces. Cases 1, 2, and 3 have the lower Eötvös numbers, and therefore exhibit the least deformation. As the Eötvös number increases further $(30 \leq$ Eo $\leq 100$ ), the bubble experiences more deformation, yielding to skirted bubbles as indicated in Figure 4.12 where the instantaneous bubble shapes captured by LBM and LSM are shown for the same non-dimensional time T. Case 6 has the highest Eötvös number and therefore undergoes more deformation, causing the bubble to break. 

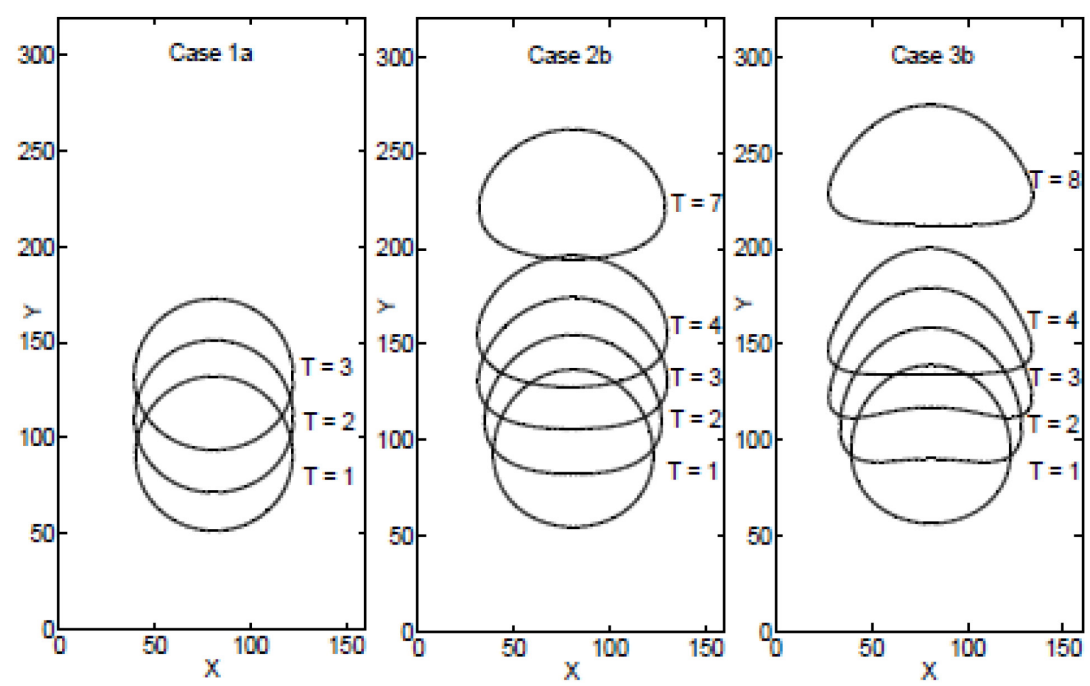

Figure 4.11. Bubble shape evolution for cases $1 \mathrm{a}, 2 \mathrm{~b}$, and $3 \mathrm{~b}$.

After the breakup, small satellite bubbles trail the main bubble, which eventually attains a spherical cap shape. Figure 4.12 shows the bubble shapes simulated with LBM and LSM in comparison for $E_{o}=5,10,30,75$, and 100. It was found that bubbles stayed intact for $E o<75$ while bubble break-up was observed for the last two cases. For the cases with no break-up, the bubbles reached the terminal shape and attained a terminal velocity; however, for the cases with break-up, the bubble shape evolved continuously. 

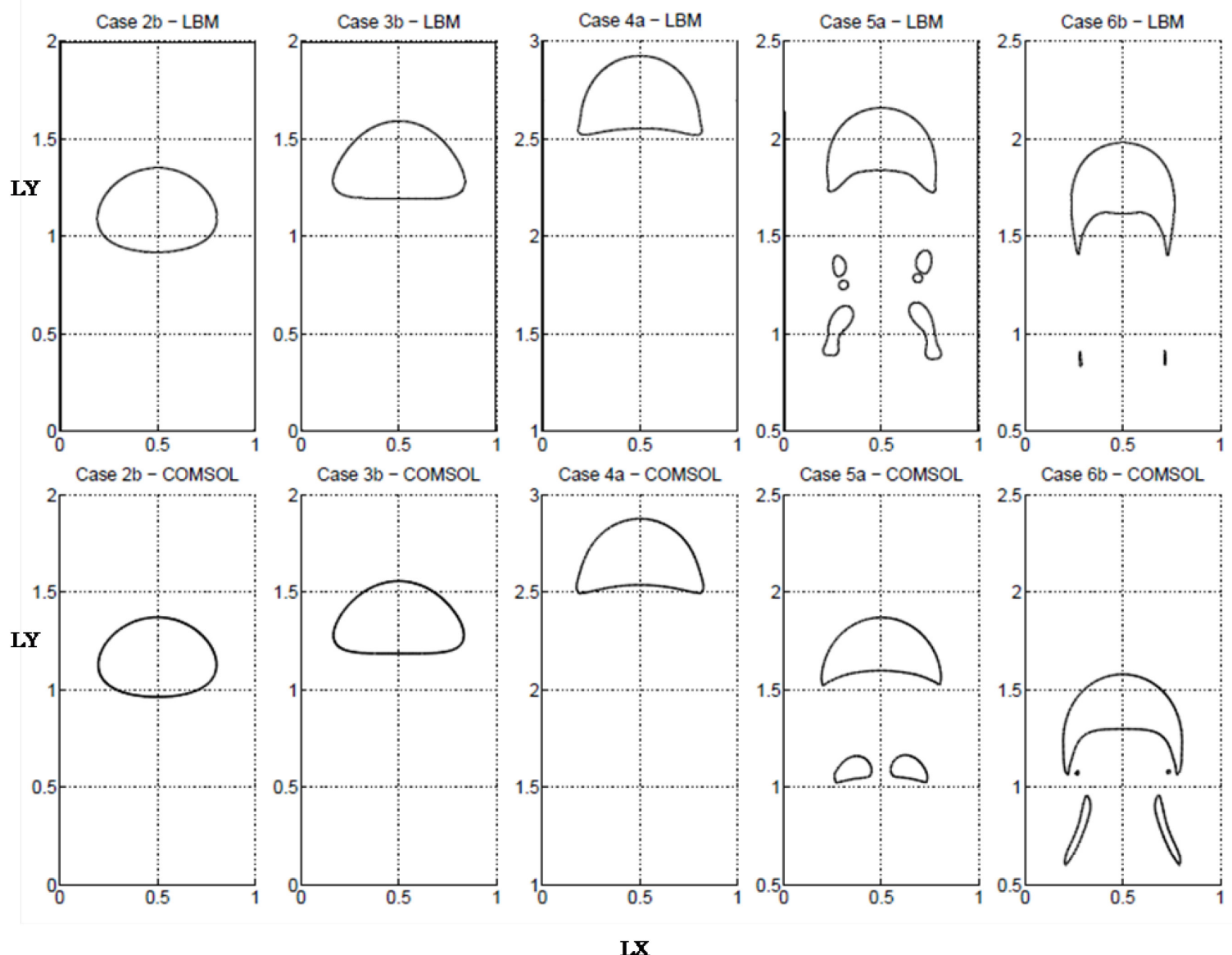

Figure 4.12. Interfacial profile of rising bubbles simulated with LBM (top) and LSM (bottom). 


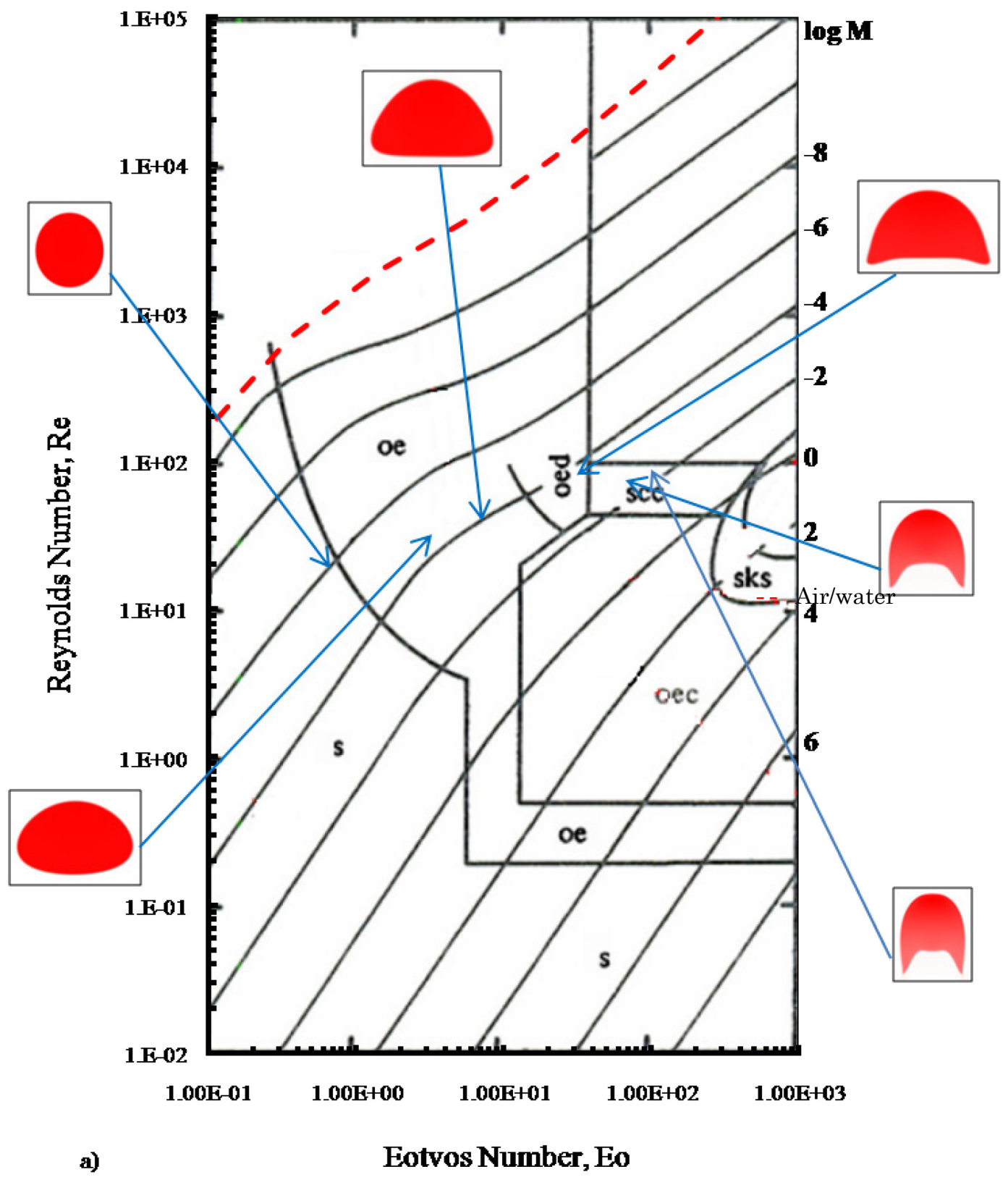




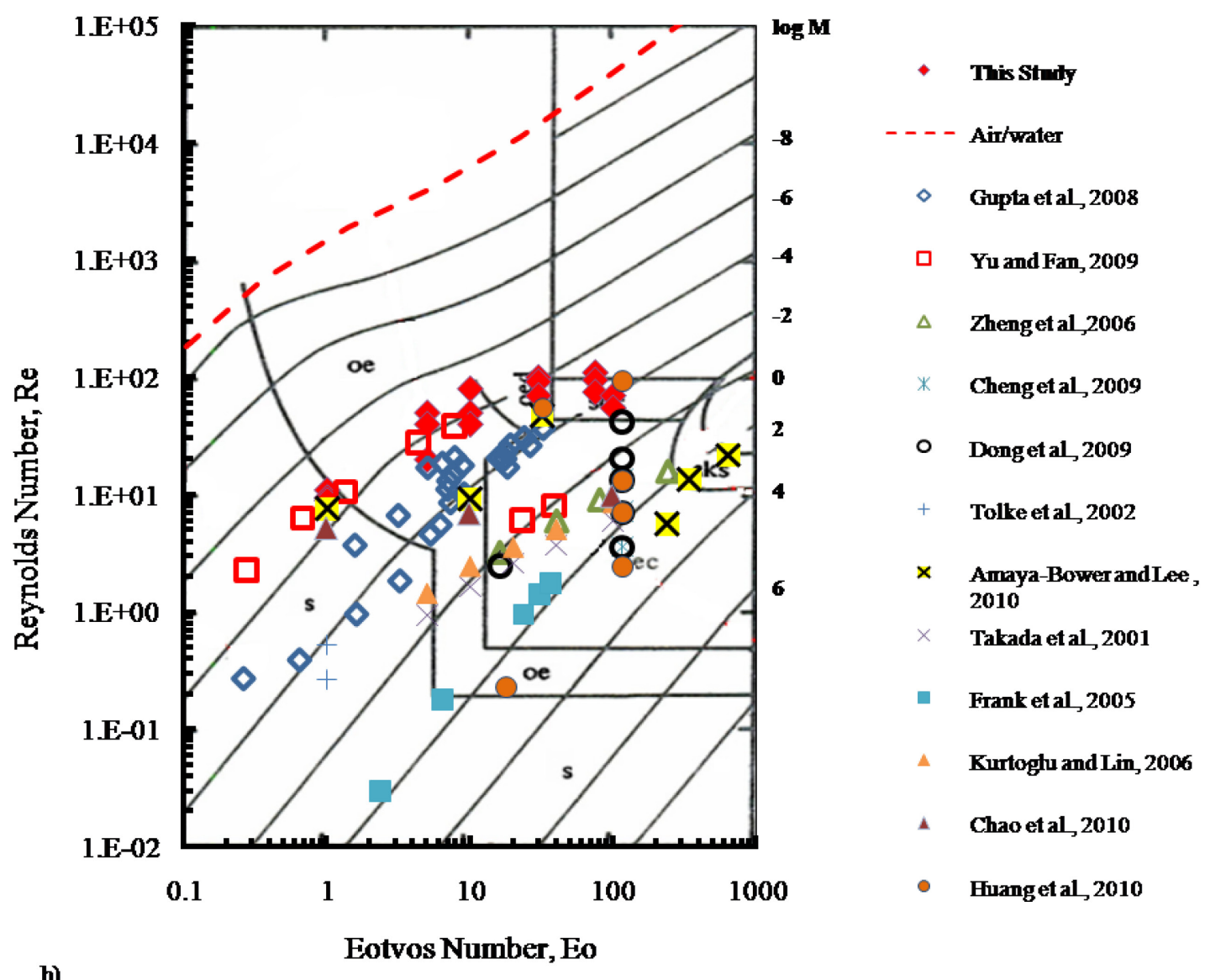

Figure 4.13. a) The images in the inset are some results of the numerical simulations using Lattice Boltzmann method; b) Bubble shape regime of my work along with other available results. s: spherical; oe: oblate ellipsoid; oed: oblate ellipsoidal (disk-like and wobbling); oec: oblate ellipsoidal cap; scc: spherical cap with closed steady wake; sco: spherical cap with open, unsteady wake; sks: skirted with smooth, steady skirt; skw: skirted with wavy, unsteady skirt [13]. The dashed line represents the Air/water system obtained with the Fan-Tsuchiya correlation [43].

Figure $4.13 \mathrm{a}$ indicates that the bubble shapes predicted by our method are consistent with the bubble shape map proposed by Clift et al. [12] , and our simulations give good qualitative and quantitative results. These results 
extend the range of previously simulations and are closer to the air/water system (Figure 4.13b).

Figure 4.14 shows the terminal Reynolds number and circularity (see Appendix A for more terminal Reynolds) of the bubble obtained with both LBM and LSM. It was observed that the bubbles reached the terminal velocity after about $\mathrm{T}=5$ for the cases without break-up.

Table 2 summarizes the terminal velocity and Reynolds numbers for cases 2 , 3, and 4 obtained with LBM and LSM. A maximum difference of $8.7 \%$ was found between the results obtained with LBM and LSM. To investigate the discrepancy between LBM and LSM, the mass conservation of the two methods was investigated by calculating the instantaneous bubble area during the rising process.


Figure 4.14. Terminal Reynolds number and circularity simulated with LBM and LSM. 


\begin{tabular}{cccc}
\hline Case & $\operatorname{Re}(\mathrm{LBM})$ & $\operatorname{Re}(\mathrm{LSM})$ & Difference (\%) \\
\hline \hline 2a & 14.70 & 16.00 & 8.13 \\
2b & 10.30 & 10.84 & 4.98 \\
2c & 7.21 & 7.90 & 8.73 \\
3a & 19.76 & 20.38 & 3.04 \\
3b & 14.22 & 14.51 & 2.00 \\
3c & 10.39 & 11.09 & 6.31 \\
4a & 43.00 & 40.41 & 6.41 \\
4b & 30.50 & 28.59 & 6.68 \\
4c & 22.83 & 21.52 & 6.09 \\
\hline
\end{tabular}

Table 2. Terminal Reynolds number obtained with LBM and LSM.

\subsubsection{Wall Effects on Final Shape and Terminal Velocity of Single Rising Bubble}

In a fully-walled domain, bubble shape and rising velocity are affected by the walls of the domain. The magnitude of these effects depends on the ratio of the bubble diameter to the width of the domain. Simulations were carried out to analyze this effect and comparison was made with both analytical solution as proposed by Krishna et al. [19] and with the criterion discussed by Clift et al. [12], who showed that the bubble rise velocity is independent of wall effects when the ratio of the bubble diameter to the width of the domain is smaller than 0.125 . In order to quantify the wall effects, I considered an ellipsoidal regime where Eötvös number, $E_{0}=5$ and Morton number, $M=9.34 \times 10^{-5}$. For this investigation, various ratios of bubble diameter 
$\left(d_{o}=30\right)$ to the width of the domain $r^{*}=d_{o} / W$ were chosen as in Table 3.

Figure 4.15 displays the effect of domain diameter on bubble shape and rise velocity at time $\mathrm{T}=6.3$ for cases $\mathrm{b}, \mathrm{g}$ and $\mathrm{i}$. As shown, as the transverse domain size decreases, the wall effect on the bubble behavior becomes more significant.

\begin{tabular}{cccccccccc}
\hline Case & $\mathrm{a}$ & $\mathrm{b}$ & $\mathrm{c}$ & $\mathrm{d}$ & $\mathrm{e}$ & $\mathrm{f}$ & $\mathrm{g}$ & $\mathrm{h}$ & $\mathrm{i}$ \\
\hline \hline $\boldsymbol{W}$ & 270 & 240 & 225 & 200 & 175 & 150 & 125 & 100 & 75 \\
$\boldsymbol{W}$ & 9 & 8 & 7.5 & 7 & 6 & 5 & 4 & 3 & 2 \\
$\boldsymbol{d}_{\boldsymbol{o}}$ & & & & & & & & & \\
\hline
\end{tabular}

Table 3. Parameters used to investigate the wall effects.
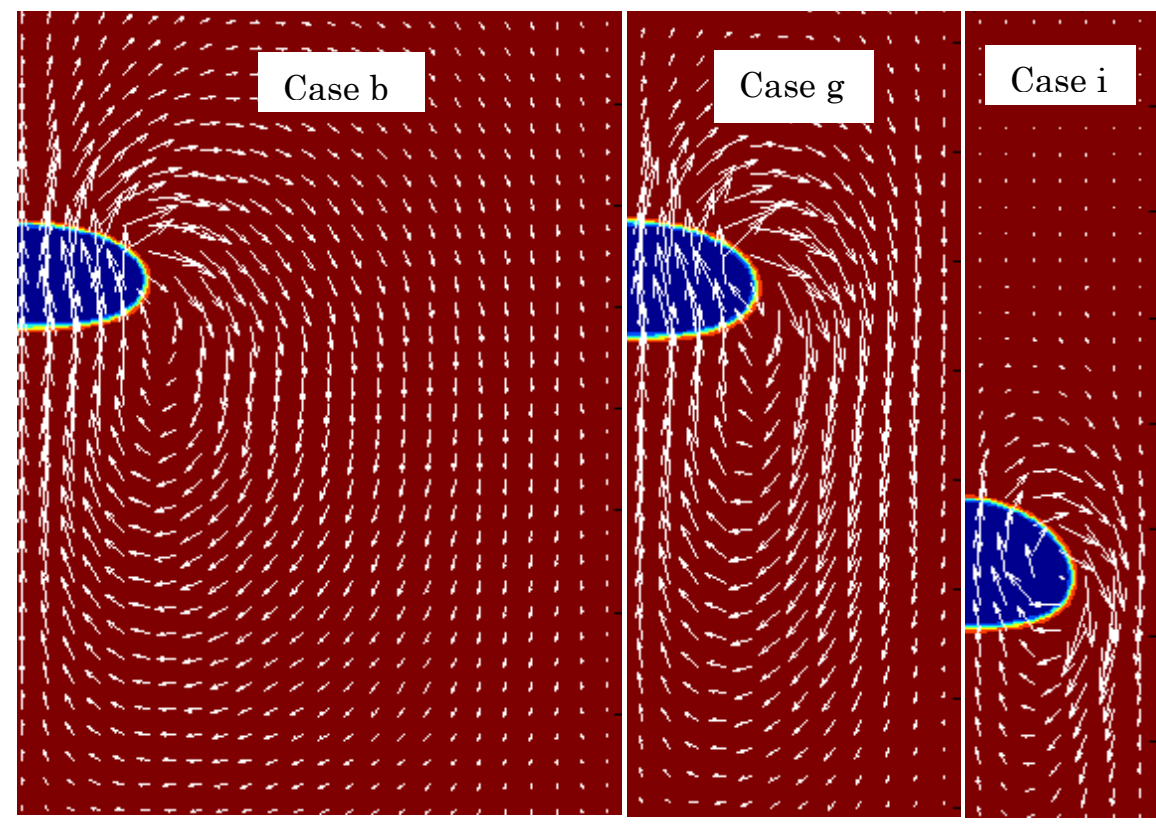

Figure 4.15. Velocity vectors and final shape for ellipsoidal bubbles at $\mathrm{T}=$ $6.30, E o=5$ and $M=9.34 \times 10^{-5}$. The right-hand boundaries represent walls. 
The viscous effect due to the wall will increase the drag between the liquid and the bubble, causing the bubble to slow down and attain its final state faster. The front of the bubble appears to be less convex (looking from the inside) in large domains. The minimum domain size for which the bubble behavior is independent of the wall effect agrees well with the Clift et al. [12] criterion $\left(r^{*} \leq 0.125\right)$. Figure 4.16 shows the wall effect on the terminal Reynolds number. The results indicate that bubbles in large domains rise freely from wall effects. However, because of computational cost it is necessary to find a domain size that provides a compromise between computational cost and accuracy.

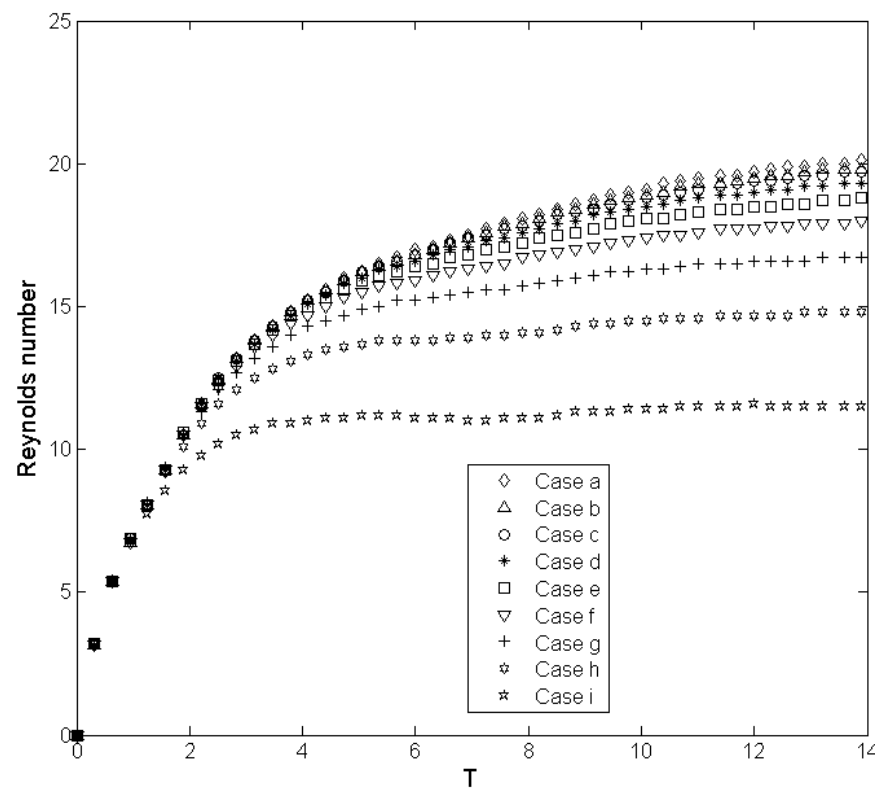

Figure 4.16. Wall effects on terminal Reynolds number, $E o=5$, and $M=9.34 \times 10^{-5}$. 
As indicated in Table 4 , case e $\left(r^{*}=0.171, W \sim 6 \mathrm{do}\right)$ should be a good compromise with a relative difference of $6 \%$ as compared to the largest domain.

\begin{tabular}{cccccccccc}
\hline Case & $\mathrm{a}$ & $\mathrm{b}$ & $\mathrm{c}$ & $\mathrm{d}$ & $\mathrm{e}$ & $\mathrm{f}$ & $\mathrm{g}$ & $\mathrm{h}$ & $\mathrm{i}$ \\
\hline \hline $\operatorname{Re}$ & 20 & 19.8 & 19.7 & 19.3 & 18.8 & 18 & 16.7 & 15 & 11.5 \\
$\varepsilon_{\text {rel }}$ & - & 0.010 & 0.015 & 0.035 & 0.060 & 0.100 & 0.165 & 0.250 & 0.425 \\
\hline
\end{tabular}

Table 4. Reynolds numbers and relative difference due to wall effects on single rising bubble, $E o=5$, and $M=9.34 \times 10^{-5}$.

Results indicated in the previous section with $\left(r^{*}=0.5, W=2 d_{0}\right)$ should therefore be adjusted. In order to consider the decrease in the rising velocity due to wall effects, simulations for large domain $\left(W=6 d_{0}\right)$ were carried out. Figure 4.17 indicates a decrease of about $49 \%$ for case $2 \mathrm{a}$. Table 5 shows the corrected Reynolds number for other cases, where adjustment was made accordingly.

To compare my simulation results with theoretical findings, the rise velocity of spherical caps which meet the criterion $E_{o}>40$ was evaluated. The following empirical relation of rise velocity for spherical cap bubble shows that the rise velocity is a unique function of $r^{*}[\mathbf{1 9}]$. 




Figure 4.17. Wall effects on terminal Reynolds number for case 2a. $E o=5$ and $M=1.3 \times 10^{-5}$.

\begin{tabular}{ccc}
\hline Case & Re & Re adjusted \\
\hline \hline 2a & 14.7 & 28.6 \\
$2 \mathrm{~b}$ & 10.3 & 20.1 \\
2c & 7.2 & 14.1 \\
3a & 19.8 & 38.5 \\
3b & 14.2 & 27.7 \\
3c & 10.4 & 20.3 \\
$4 \mathrm{a}$ & 43.0 & 64.5 \\
$4 \mathrm{~b}$ & 30.5 & 44.5 \\
$4 \mathrm{c}$ & 22.8 & 32.5 \\
$5 \mathrm{a}$ & 80 & 93 \\
$5 \mathrm{~b}$ & 62 & 72 \\
$5 \mathrm{c}$ & 48 & 50 \\
$6 \mathrm{a}$ & 70 & 80 \\
$6 \mathrm{~b}$ & 59 & 62 \\
\hline
\end{tabular}

Table 5. Adjusted Reynolds numbers associated with wall effects. 


$$
V_{t}=\left\{\begin{array}{cc}
0.62 \sqrt{g d_{o}}, & d_{o} / W<0.07 \\
0.62 \sqrt{g d_{o}}\left[1.1 \exp \left(-\frac{1.5 d_{o}}{W}\right)\right] & 0.07<d_{o} / W<0.4 \\
0.236 \sqrt{g W} & d_{o} / W>0.4
\end{array}\right.
$$

Simulations were carried out for $E o=75$ in a $480 \times 800$ domain for 5000 time steps. Bubble diameter, $d_{o}=80$, and $r^{*}=0.167$. Figure 4.18 compares our simulation results with Krishna-Van Baten model [19], confirming the predicted behavior for spherical cap bubbles.

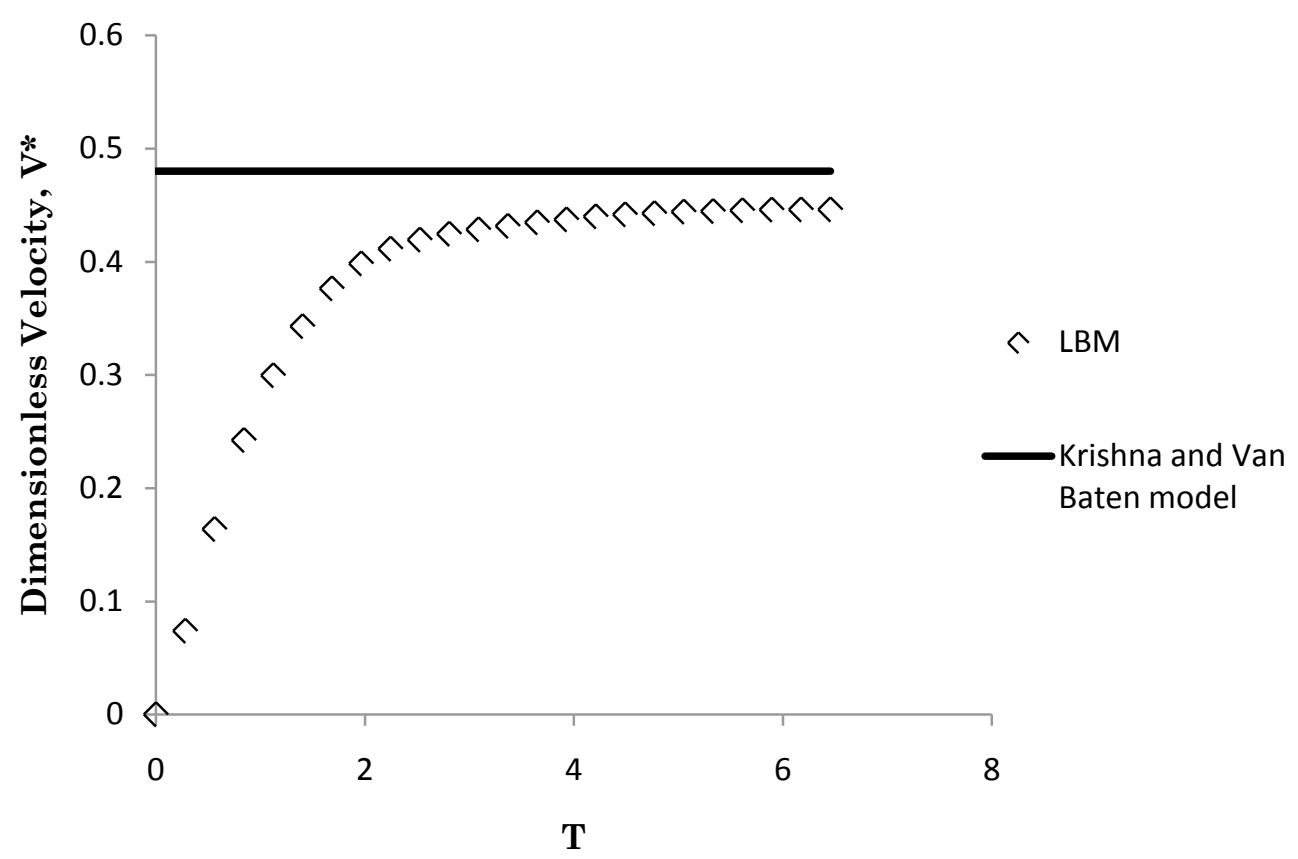

Figure 4.18. Comparison of spherical cap rise velocity with Krishna-Van Baten model for $\mathrm{r}^{*}=0.166$ and $E_{O}=75 . \boldsymbol{V}^{*}=\boldsymbol{V}_{\boldsymbol{t}} / \sqrt{\boldsymbol{g}_{\text {applied }} \boldsymbol{d}_{\boldsymbol{o}}}$.

The computational performance of the LBM method is also examined in this work. Because the two fluids simulated here were assumed to be incompressible and immiscible, the expected normalized bubble area should 
be around 1 for a perfectly mass-conserving numerical method provided the density does not change. As indicated in Figure 4.19, the change in the bubble area obtained with LBM was almost negligible whereas a large reduction in bubble area was observed in the simulations with LSM, especially for the cases with Eo $\geq 30$.


Figure 4.19. Normalized bubble area obtained with with LBM and LSM.

This effect is more prominent for the cases at $E_{O}=75$ and $E_{o}=100$ where bubble break-up was observed. The bubble rises slower with the LSM when the bubble breaks due to the reduced area of the bubble. A grid convergence study was performed to investigate the effect of grid size in LSM on the reduction of the area. Figure 4.20 shows the grid convergence study with the LSM where the results for bubble area change are shown for three different levels of grid spacing $(\Delta \mathrm{h}=0.0089,0.013,0.02)$ for case $5 \mathrm{a}$. 
A grid convergence index was calculated using the solutions at three different grid levels and the error due to discretization at the fine grid solution was found to be of $15 \%$. The effect of the grid spacing on the shape of the bubble for case $5 \mathrm{a}$ is presented in Figure 4.20b. Although the mass conservation is improved by refining the grid, the computational cost of the LSM increases substantially. For case 5a given in Figure 4.20, the fine grid simulation took 60 hours and 45 minutes compared to 12 minutes of single-processor computation time using the LBM to achieve the same bubble shape.
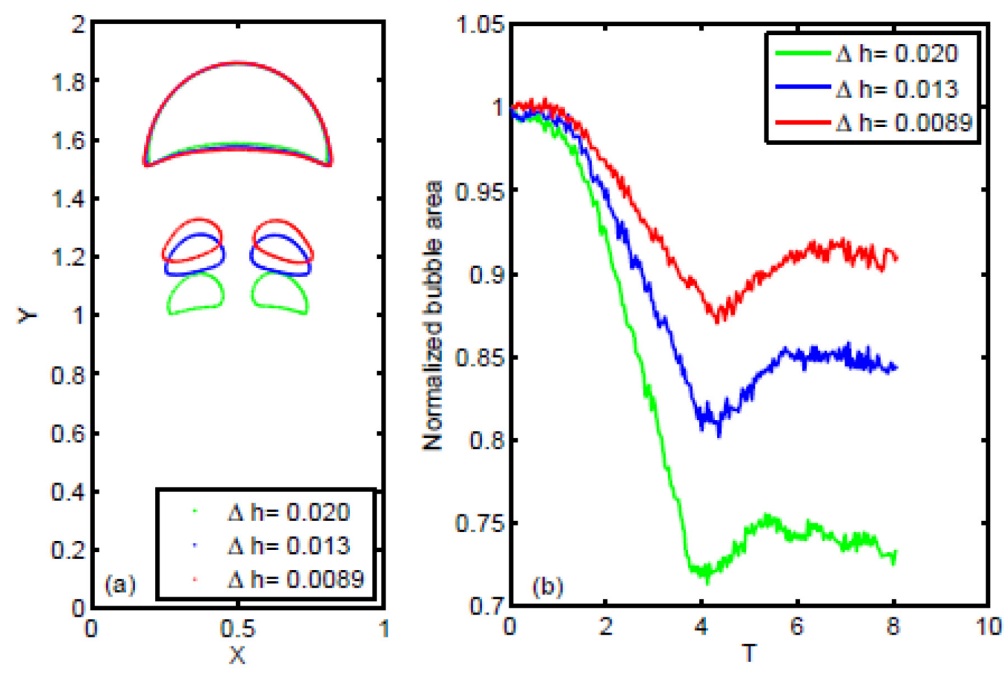

Figure 4.20. Grid convergence study for case 5a using LSM.

\subsubsection{Effects on the Flow Field}

Figure 4.21 presents the LBM results for the velocity field around the bubble, as it forms its terminal shape for different values of Eo and M. The velocity vectors are shown here with respect to the fixed coordinate system (domain 
reference frame) and the velocity is observed to be the largest at the bubble center.

The bubble interface is represented with the red lines. A wake structure forms behind the bubble for the cases where the Reynolds number was found to be larger than 40 . The internal circulation or the vortex ring structure in the wake region of the bubble was also investigated in Figure 4.22 where the pathlines were plotted by using the relative velocity with respect to the bubble. Experimental observation of wake regions was performed by Bhaga and Weber, who noticed that the wake structure is related to the degree of deformation, the terminal velocity, and the bubble size. The streamlines of the flow around the bubble are calculated in the frame of reference of the top of the bubble.

A large wake region is observed with high deformation, under high Eötvös number (i.e., higher volume), and Reynolds number. As indicated on Figure 4.22, cases 1,2 , and 3 undergo little deformation, and therefore no significant wake is produced behind the bubble. The streamlines smoothly surround the bubble. On the other hand, as Eo increases, so do the deformation and the wake. Bhaga and Weber found a critical point $\left(M=4 \times 10^{-3}\right)$ to separate closed steady and open wakes. As shown on Figure 4.22, case 4c exhibits an open wake, unlike other cases. With a Morton number of $8.14 \times 10^{-4}$, case $4 \mathrm{c}$ has probably crossed the transition point between closed and open wake. The 
wake flow circulation patterns obtained by our simulation agree well with the predictions. Similar results were obtained by Amaya-Bower and Lee [45].
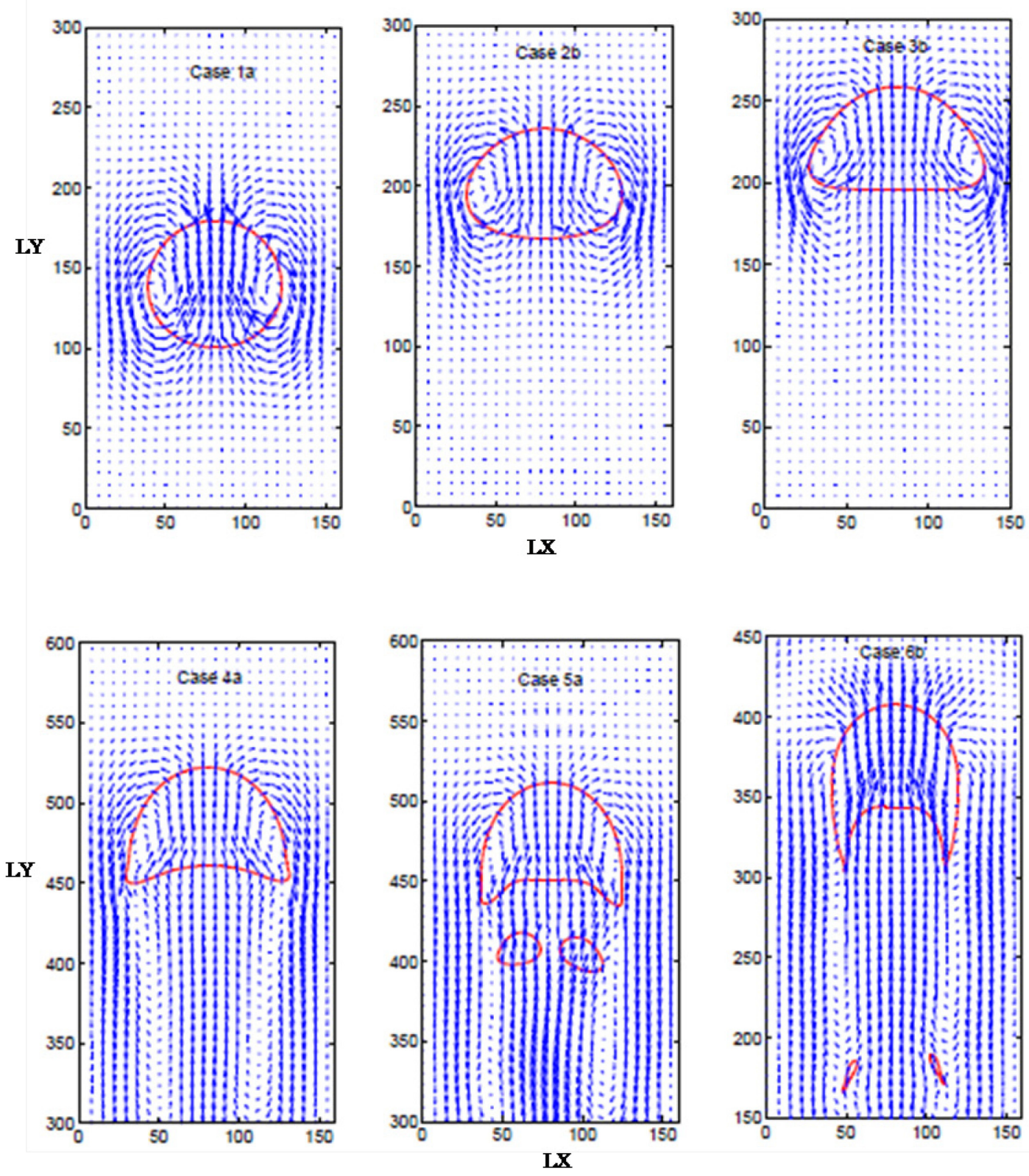

Figure 4.21. Velocity vectors of the bubble in the domain reference frame, as it forms its terminal shape for different $E_{o}$ and $M$. The case is indicated at the top of the graph, and the associated Eo and $\mathrm{M}$ can be found in Table 1. 




(a) Case $1 a$

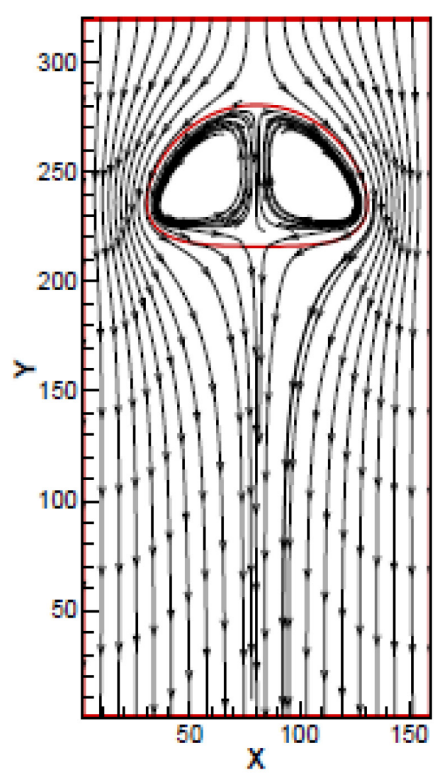

(c) Case $3 c$

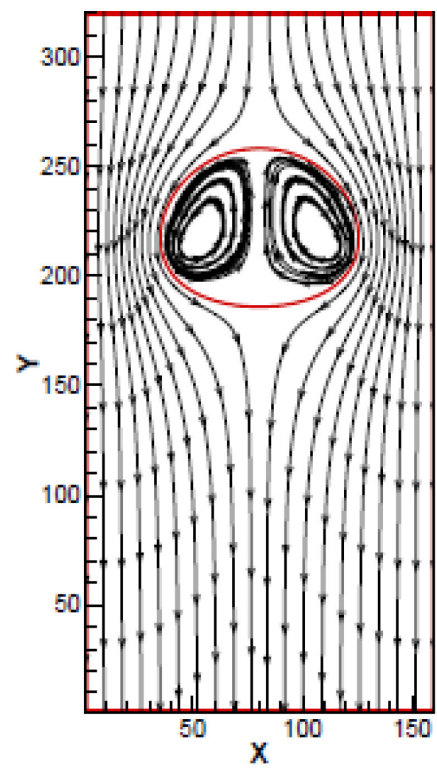

(b) Case $2 c$

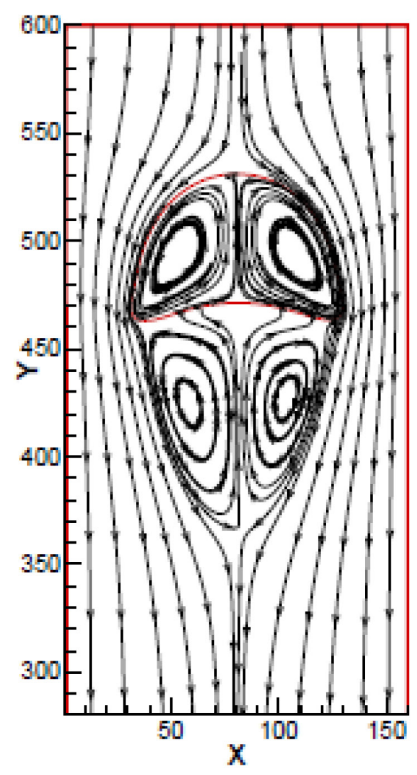

(d) Case $4 c$

Figure 4.22. Streamlines around the bubble in the reference frame at the top of the bubble, at the terminal state for different $E_{O}$ and $M$. The cases are indicated at the bottom of each graph, and the associated $E o$ and $M$ can be found in Table 1. 


\subsection{Static Bubble Simulation with Explicit Density Difference}

To ensure that the surface tension effect is properly implemented in this model, I considered two test cases. First, I checked if an initial square bubble can freely deform to a circular bubble and secondly I simulated the coalescence of two static bubbles that merge to become a single circular bubble.
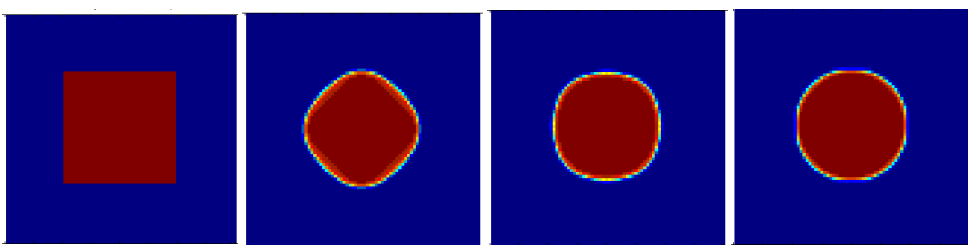

Figure 4.23. Free deformation of a static bubble from square shape to a circular shape.


Figure 4.24. Coalescence of two identical circular bubbles and free deformation to a circular shape.

Both cases show deformation to the final circular bubble, indicating that the surface tension effect is correctly implemented.

In the effective buoyancy approach described in the Section above, the density difference that controls the buoyancy was included in the gravity forcing. In order to explicitly account for density difference, the masses of components were included explicitly in the model, and a test case simulation 
of a static bubble in a liquid-filled domain were carried out for 50,000 time steps in a $100 \times 100 \mathrm{lu}^{2}$ domain with periodic boundaries. The interaction strength $\mathrm{G}$ that controls the fluid-fluid interfacial tension was 3.5, and the relaxation times were kept to 1 for both components.

Figure 4.25 shows the density profile along the centerline of the bubble. As indicated the density ratio, ${ }^{\rho_{H}} / \rho_{L}=2$, and viscosity ratio, ${ }^{\mu_{H}} / \mu_{L}=2$

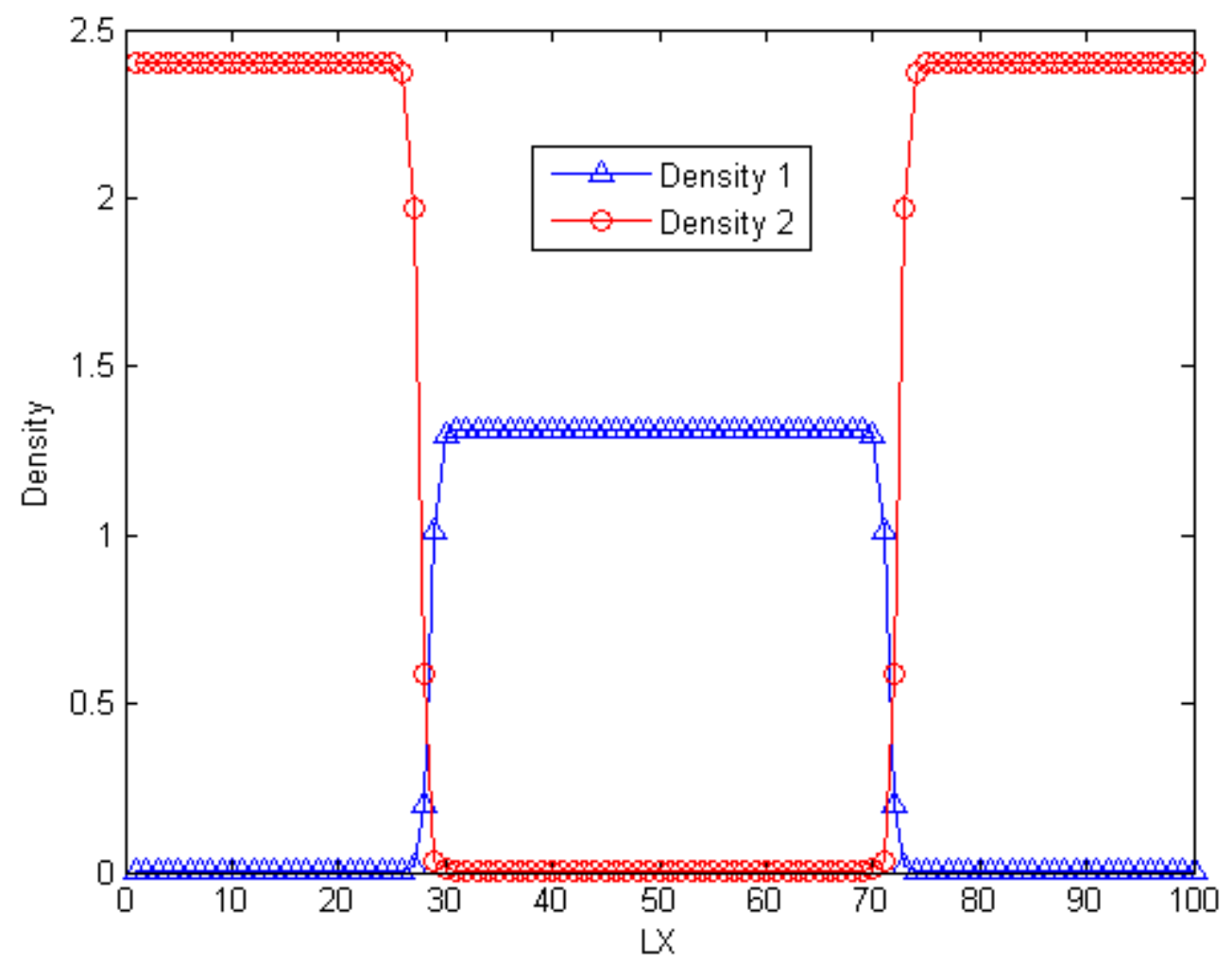

Figure 4.25 : Density profile along the centerline of the bubble. The lighter gas is surrounded by the heavy liquid.

A real immiscible system may present a sharp interface. The interface thickness, where the transition occurs $(\sim 5$ lattice nodes) proves that the 2 
components are not exactly immiscible. However, this interface thickness has been reported by other investigators [ [71], [7] ]. Because of this miscibility at the interface, the mean density in each phase is slightly different from the initial density.

\subsection{Multiple Bubbles Simulation}

Brereton and Korotney [72] presented experimental observations of interactions between two gas bubbles rising in an initially quiescent liquid. Their experiment on bubble coalescence reveals a big difference in shape between the leading and trailing bubbles. In fact, the interaction and the coalescence of gas bubbles are characterized by three distinct stages. The first describes the initial contact (collision) between the bubbles. The second stage is the liquid drainage, where the liquid film between the gas-bubbles is squeezed out and ruptured, leading to the third stage where the coalescence occurs. The goal in this study is to capture these features for different scenarios which will help to better understand the coalescence characteristics.

We first consider interactions between identical adjacent bubbles $\left(d_{o}=23 \mathrm{lu}\right)$, placed in the same horizontal plane and separated by 3 times their diameter. Simulations were carried out in a $120 \times 300$ fully-walled domain. The density ratio, $\rho_{H} / \rho_{L}=2$. Figure 4.26 shows that, in this configuration, each bubble rises independently, and at the velocity it would have had if it was rising 
alone in the domain. It can be seen that the two bubbles do not coalesce; instead the bubbles tend to avoid each other. The complex structure of the wake trailing the bubbles reveals a down flow of liquid between the bubbles, keeping them away from each other, and therefore, preventing them from coalescing.

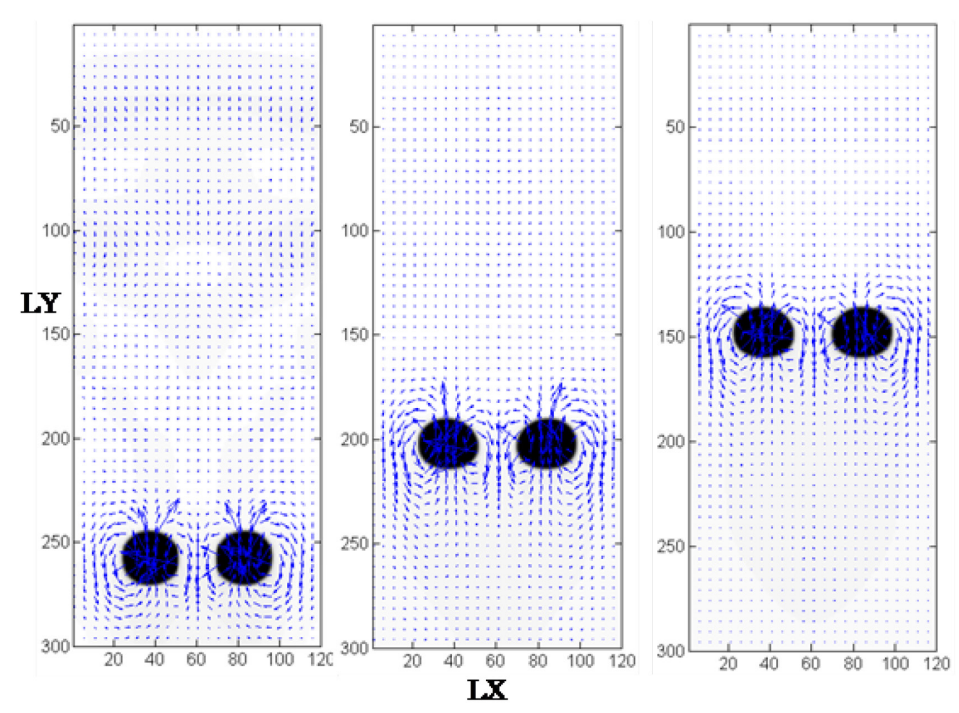

Figure 4.26. Bubble shape and velocity fields of two identical bubbles of diameter $23 \mathrm{lu}$. Initially the bubbles are on the same horizontal plane and separated by $3 d o$. Snapshots are taken at T $=2.10,7.57,13 . \quad E o=1.6$, and $M=2.6 \times 10^{-4}$.

In order to study the wake effect of the leading bubble on the trailing bubble, we first consider a scenario of two identical and co-axial bubbles of diameter, $d_{o}=30 \mathrm{lu}$, aligned vertically and separated by 3 times their diameter. Figure 4.27 shows that in this case $(E o=5)$, the leading bubble experiences much more deformation than the trailing bubble. In fact the leading bubble seems 
to freely rise in the surrounding fluid and the wake it generates considerably affects the dynamics of the lower bubble. The wake of the leading bubble behaves as a "lift" for the trailing bubble and therefore reduces its drag, hence its deformation. As time goes on, the trailing bubble rises in the wake region of the upper bubble, and the distance between the two bubbles decreases rapidly, leading to collision $(\mathrm{T}=8.42)$ and soon after, to coalescence $(\mathrm{T}=8.84)$. The resulting coalesced bubble is two times larger than the initial bubble, and the shape agrees very well with the flow regime.
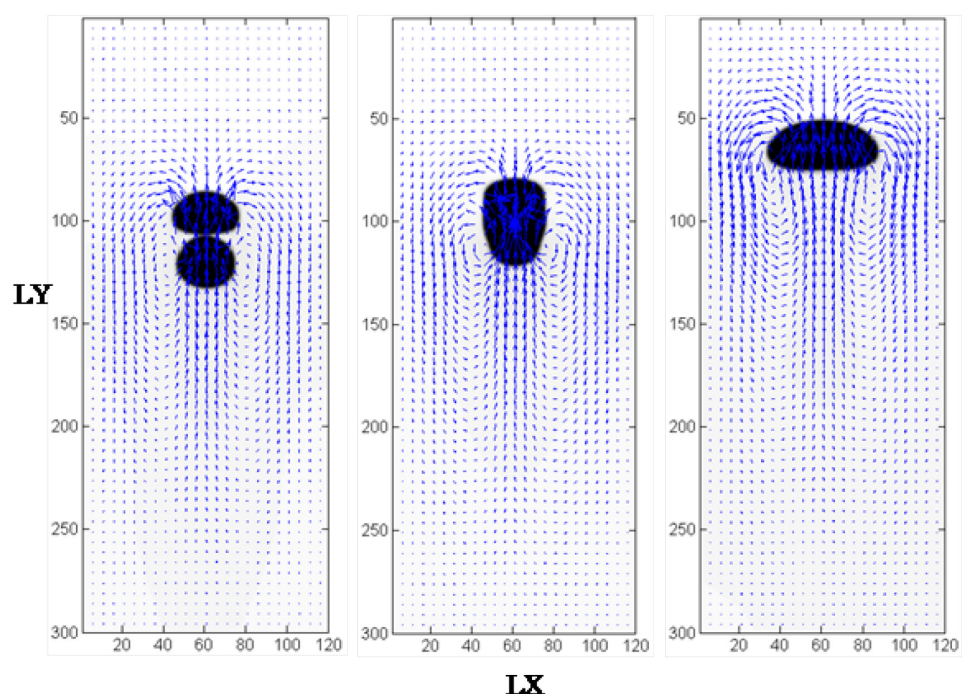

Figure 4.27. Bubble shape and velocity fields of two identical bubbles of diameter $30 \mathrm{lu}$. Initially the bubbles are separated by $3 d o$. Collisions occurs at $\mathrm{T}=8.42$, the coalescence at $\mathrm{T}=8.84$ and bubble attains its terminal ellipsoidal shape at 10.95. Eo $=4$, and $M=5.63 \times 10^{-4}$.

Figure 4.28 shows the dynamics of two co-axial bubbles of different diameter, aligned vertically, and rising in a continuous liquid phase. As compared to 
results in Figure 4.27, the leading bubble is much bigger. The inertia of the fluid behind the leading bubble is much stronger and therefore causes the coalescence to happen faster as compared to Figure 4.27. As indicated on Figure $4.28 \mathrm{c}$, a small liquid drop has been trapped in the gas phase after the coalescence, because of the inertia during the collision. Soon after, the encapsulated liquid drop "escapes" the bubble from the bottom (Figure 4.28d) and the final shape naturally emerges. The results agree very well with the experimental observations by Brereton and Korotney as reported in [25]. Takada et al. [41] and Gupta and Kumar [7] instead, used three identical and small bubbles and observed the same phenomena.



a

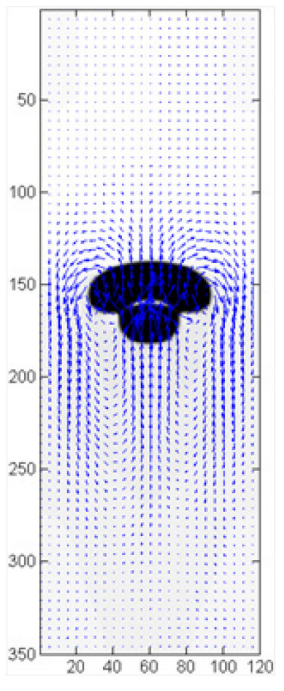

b

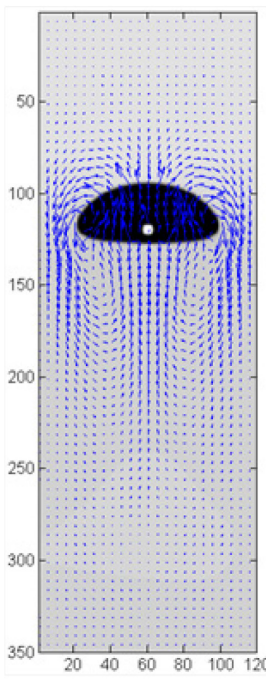

IX



d

Figure 4.28. Bubble shape and velocity fields of two co-axial bubbles in a domain of $120 \times 350$ lattice nodes. Initially, the leading bubble has a diameter of $40 \mathrm{lu}$ and the trailing bubble $30 \mathrm{lu}$. Collision occurs at $\mathrm{T}=7.92$, coalescence at $\mathrm{T}=7.30$. The encapsulated liquid drop is shown at $\mathrm{T}=10.59$. The last snapshot shows a bump at the bottom of the bubble indicating the escape of the liquid drop. 
The second configuration here considers two identical bubbles of same size and non co-axial. Their centers are initially separated by a distance of 3 times the bubble radius in the vertical direction. We performed simulations for $E_{o}=$ 16 and $M=2 \times 10^{-4}$. Figure 4.29 compares our results with those obtained by Annaland et al. [21]. As in the case of co-axial bubbles, the leading bubble seems to freely rise in the surrounding liquid. The trailing bubble is affected by the leading bubble, and experiences more deformation when it enters the wake region behind the leading bubble.

\begin{tabular}{ccc}
\hline Parameters & Annaland et al. [21] & This study \\
\hline \hline$\rho_{H} / \rho_{L}$ & 100 & 2 \\
$\mu_{H} / \mu_{L}$ & 100 & 2.8 \\
$E_{O}$ & 16 & 16 \\
$M$ & $2 \times 10^{-4}$ & $2 \times 10^{-4}$ \\
$d_{0}$ & $0.01 \mathrm{~m}$ & $43 \mathrm{lu}$ \\
$\sigma$ & 0.1 & 0.2 \\
\hline
\end{tabular}

Table 6. Parameters used for the oblique coalescence. 

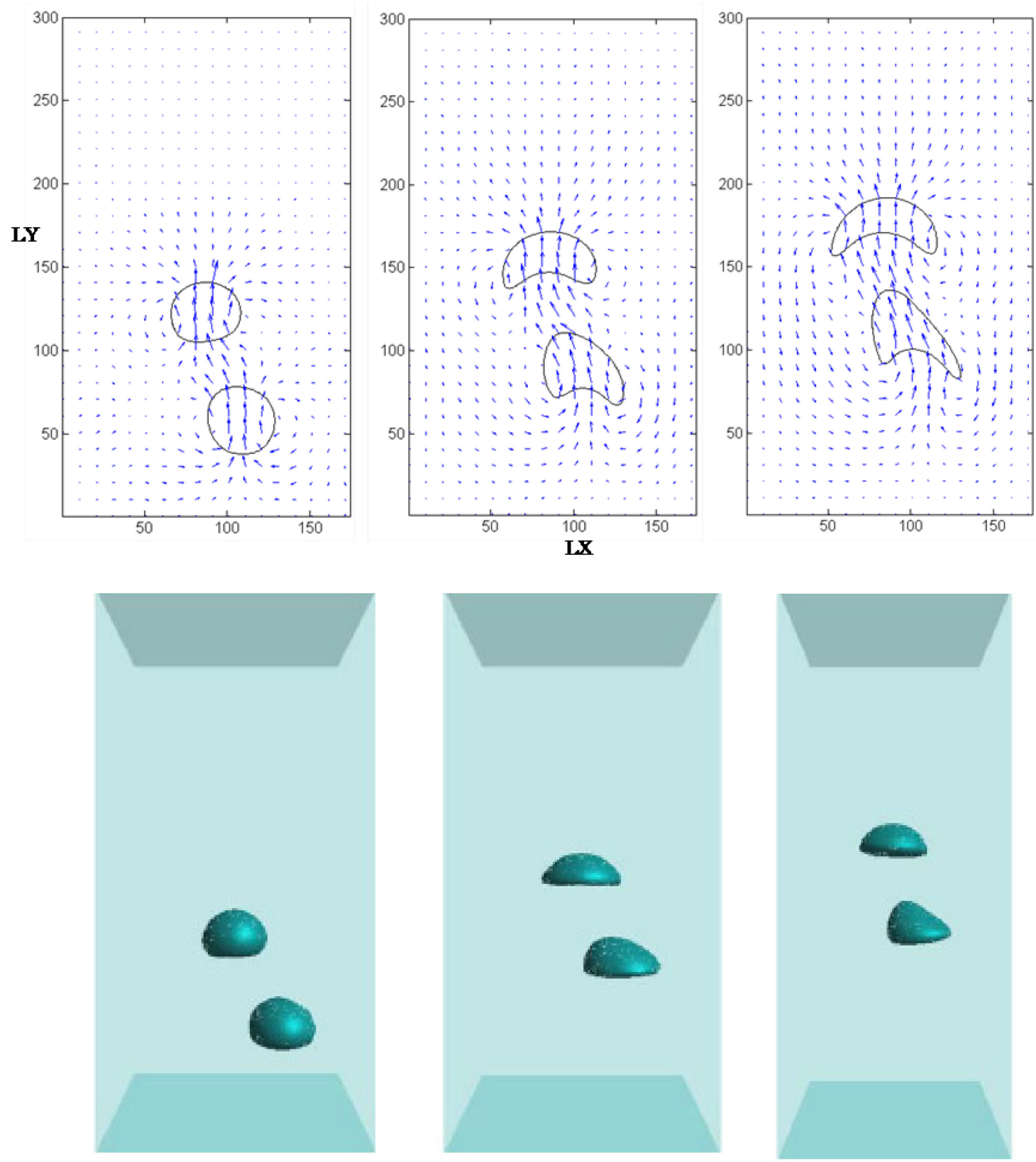

Figure 4.29. Bubble shape and velocity fields of two oblique bubbles in a domain of $174 \times 300$ lattice nodes. $E o=16$ and $M=2 \times 10^{-4}$. The top snapshots represent the LBM results. Bubble diameter $=108 \mathrm{lu}$. From left to right, the snapshots correspond to $\mathrm{T}=1.17,2.75$ and 3.35 . The bottom snapshots show results obtained by Annaland et al. [21] in a $80 \times 80 \times 160$ grid size. 
The difference between the two methods might be the result of the dimensions of the system and the difference in the density and viscosity ratios. Despite this difference in shape deformation, the simulations results capture the flow features very well.

\subsection{Bubble Flow through Porous Media}

Lattice Boltzmann simulations of immiscible flow through porous media have recently been investigated by Tolke et al. [10], and based on the model proposed by Gunstensen et al. [28]. Although LBM has been applied to simulate liquid-vapor interface configuration in porous media [73], no such investigation has been done in complicated peatland environments where a gas bubble such as methane $\left(\mathrm{CH}_{4}\right)$ can build up, escape, and be released to the atmosphere by ebullition. Ebullition is supposed to occur when the buoyancy of bubbles overcomes mainly the surface tension forces that keep them in place [3].

The release of these $\mathrm{CH}_{4}$ bubbles from the peat is most likely affected by the way they get trapped within the peat. Therefore interaction between bubbles and the surrounding environment (pore water, peat internal structure) needs to be taken into account. In addition, parameters such as pressure, temperature, and microorganisms have to be considered to analyze the implications for gas bubble volume and production rate. 
In this section I investigate the ability of the SC-type LBM for multiphase flows through porous media. Although considerable experimental effort has been made to investigate gas generation such as methane fluxes from peatland ecosystems, questions remain on the constraints that affect the production rate of this strong greenhouse gas [4]. Besides, only a few simulations involving CFD have been devoted to such investigations to support existing experimental and semi-empirical findings [ [4], [74], [75], [76]]. Because the buildup and the release of $\mathrm{CH}_{4}$ depend on the production rate and the location of the sources in the peat, at this point of my research, my main emphasis was on the implementation of source terms for gas bubbles and their interaction inside porous media and then, to provide information enabling permanent incorporation into the LBM code.

\subsubsection{Line Source Generation}

A line source of bubbles were generated using single component multiphase SC-type LBM. As indicated on Figure 4.30, the source is located at the bottom of a domain of $100 \times 100$ lattice nodes. Constant and equal pressure was specified on the north and south boundaries of the domain for both components. East and west boundaries were considered periodic. I initially start with uniform density distribution and equally-spaced nucleation was produced at each lattice node at the bottom of the domain (Figure 4.30b). I assume zero order kinetics, i.e., the rate of mass production with time is 
constant. One can notice that the bubbles rise because of gravity, interact, and coalescence to form bigger bubbles. Quantitative analysis that examines the physical properties of the peat will be considered in future works. Details on the implementation of the simulation approach can be found in Appendix

A.

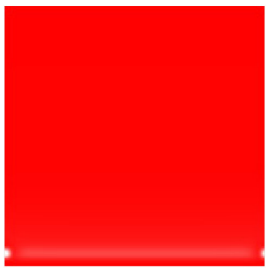

a

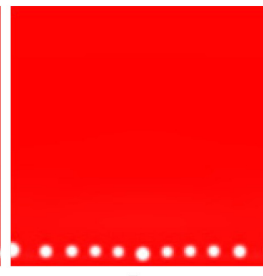

b



c



d

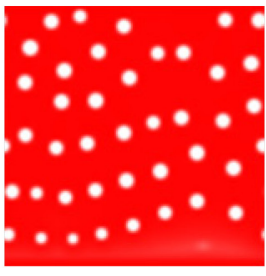

e

Figure 4.30. Line source generation in a multicomponent system and assuming a zero order kinetic. Frames from left to right: 1000, 1500, 8000, 10000,20000 time steps in a $100 \times 100 \mathrm{lu}^{2}$ domain. $\mathrm{g}=1.26 \times 10^{-6}$.

\subsubsection{Random Source Generation}

In real cases, the bubbles may not be generated as a line source. Instead, uniform random generation throughout the domain or some intermediate source configuration between a line source and a uniform source is likely to occur. Also assuming a zero order generation rate, a source term was randomly simulated in a $100 \times 100$ lattice node domain as illustrated on Figure 4.31. More details on how this simulation has been implemented and incorporated into my code can be found in appendix A. 



Figure 4.31. Multicomponent random nucleation. Frames from left to right: $1000,1500,2000,10,000$ time steps in a $100 \times 100 \mathrm{lu}^{2}$ domain. $\mathrm{g}=1.26 \times 10^{-5}$.

The previous results account only for pure fluid systems with no interactions with solid surfaces encountered in peat. Incorporation of solid surfaces requires simulation of fluid-solid interaction force and interfacial tension between the different fluid components and the solids. The surface force between solid surface and each component is computed as [62]:

$$
F_{a d s}^{\kappa}(\boldsymbol{r}, t)=-G_{a d s}^{\kappa} \rho^{\kappa}(\boldsymbol{r}, t) \sum_{a} \omega_{a} s\left(\boldsymbol{r}+\boldsymbol{e}_{a} \delta t\right) \boldsymbol{e}_{a}
$$

The $s\left(\boldsymbol{r}+\boldsymbol{e}_{a} \delta t\right)$ function takes the value 1 or 0 to indicate the presence or not of solid, respectively. The parameter $G_{a d s}^{\kappa}$ is the interaction strength between each component and the solid, and should be kept as low as possible for the wetting fluid.

The contact angle in fluid 1 (bubble) is given by the Young's equation as follows [62]:

$$
\cos \theta_{1}=\frac{\sigma_{s 2}-\sigma_{s 1}}{\sigma_{12}}
$$


This equation incorporates the interfacial tensions between the two components, $\sigma_{12}$, and between each component and the solid surface, i.e., $\sigma_{s 1}$ and $\sigma_{s 2}$.

As in Sukop and Thorne [62], the interfacial tensions in Eq. (4.8) are substituted by their corresponding adhesion strengths. Later on, Huang et al., [34] proposed a simple equation to approximate the contact angle in the SC LBM scheme:

$$
\cos \theta_{1}=\frac{G_{a d s}^{2}-G_{a d s}^{1}}{G_{12} \frac{\rho_{A}-\rho_{B}}{2}}
$$

where $\rho_{A}$ is the main equilibrium density, and $\rho_{B}$ the dissolved density associated to each fluid. Simulations for various contact angles were carried out on $300 \times 50$ lattice nodes. From an initial condition of a $30 \times 30 \mathrm{lu}^{2}$ square of fluid 1 (in white) surrounded by fluid 2 (in red), runs were performed for 20,000 time steps to allow stable interfacial tension between the fluids and the solid surface.

Table 7 gives the adhesion parameters used to compute the different contact angles from Eq. (4.9). The image processing software Image $[$ [77] was used to measure the contact angles (see Appendix E) and results are also shown in

Table 7 for comparison. Figure 4.32 illustrates different contact angles for multicomponent fluids interacting with the solid surface. 


\begin{tabular}{cc|c|c|c}
\hline \multirow{2}{*}{$G_{\text {ads }, 1}$} & \multirow{2}{*}{$G_{\text {ads,2 }}$} & \multicolumn{2}{|c|}{$\begin{array}{c}\text { Contact angle } \\
\text { (in degree) }\end{array}$} & \multirow{2}{*}{$\varepsilon_{\text {rel }}(\%)$} \\
\cline { 3 - 4 } & & LBM & Image J & \\
\hline \hline-0.5 & 1.246 & 0 & - & - \\
-0.7 & 0.986 & 15 & 14.1 & 6.3 \\
-0.05 & 1.463 & 30 & 28.6 & 5 \\
-0.05 & 1.185 & 45 & 43.3 & 4 \\
-0.05 & 0.823 & 60 & 60.2 & 0.3 \\
-0.05 & -0.05 & 90 & 89.9 & 0.1 \\
0.5 & -0.373 & 120 & 123.5 & 2 \\
0.5 & -0.736 & 135 & 135.4 & 0.3 \\
1 & -0.515 & 150 & 153 & 2 \\
0.875 & -0.875 & 180 & - & - \\
\hline
\end{tabular}

Table 7. Adhesion parameters and contact angles as obtained with LBM and ImageJ. The last column gives the relative difference between the two approaches.

To illustrate bubble gas generation and flow in porous media, we tested the ebullition of a line source term through solid obstacles as in Figure 4.33. Unless otherwise mentioned, the fluid-fluid interaction strength $G_{12}=3.5$ was used. Three cases with 15 degree, 90 and 180 degree contact angles were performed in order to capture the fluid-solid interaction effects on the fluid flows. The adhesion parameters for these cases are those given in Table 7 . 

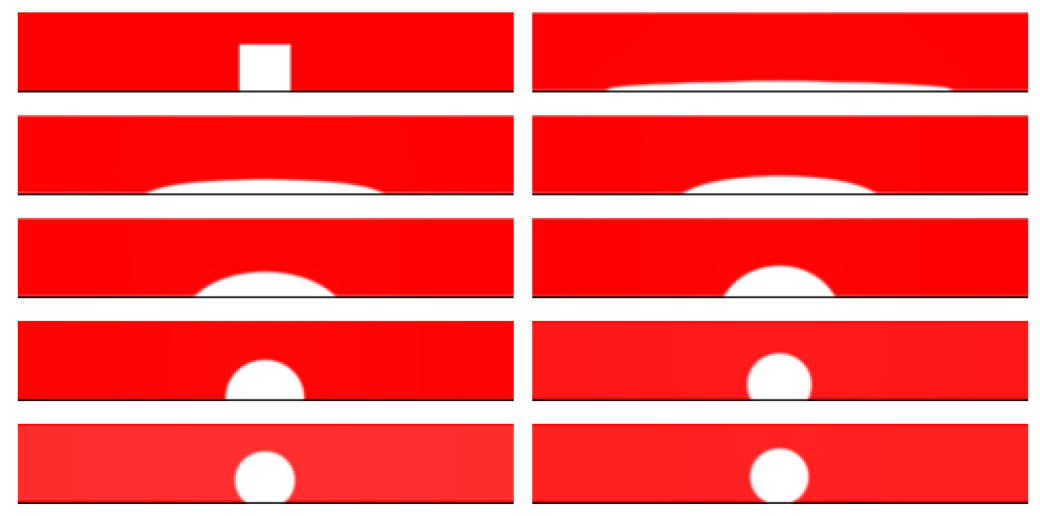

Figure 4.32. Interaction of multicomponent fluids with solid surfaces for different contact angles with fluid 1 (in white). Snapshots represent 20,000 time steps from an initial condition of a $30 \times 30 \mathrm{lu}^{2}$ square of fluid 1 surrounded by fluid 2 (in red) in a $300 \times 50$ lattice nodes domain. Each region is associated with density $\rho_{A}=1$ and a dissolved density $\rho_{B} \sim 10^{-3}$ at equilibrium. Images from left to right and top to bottom: Initial condition, $0^{0}$, $15^{0}, 30^{\circ}, 45^{\circ}, 60^{\circ}, 90^{\circ}, 120^{\circ}, 135^{\circ}$, and $150^{\circ}$.

The results of applying these adhesion strength values are illustrated on Figure 4.33 for comparison. As shown, the fluid-solid interfacial tension plays a major role on the flow. Low contact angle hinders the gas ebullition since the fluid completely wet the solid, whereas for 180 degree contact angle, the gas avoids the surface and rises much freely. It can also be seen that, bubbles get trapped and buildup at the bottom of the surface until there is enough mass to overcome the force hindering their motion. However, this change in volume may not be only due to mass buildup but, also to atmospheric pressure as reported by Strack et al. [75] and Tokida et al. [76]. Their observations suggested that ebullition most likely occurs with reductions in the atmospheric pressure. 

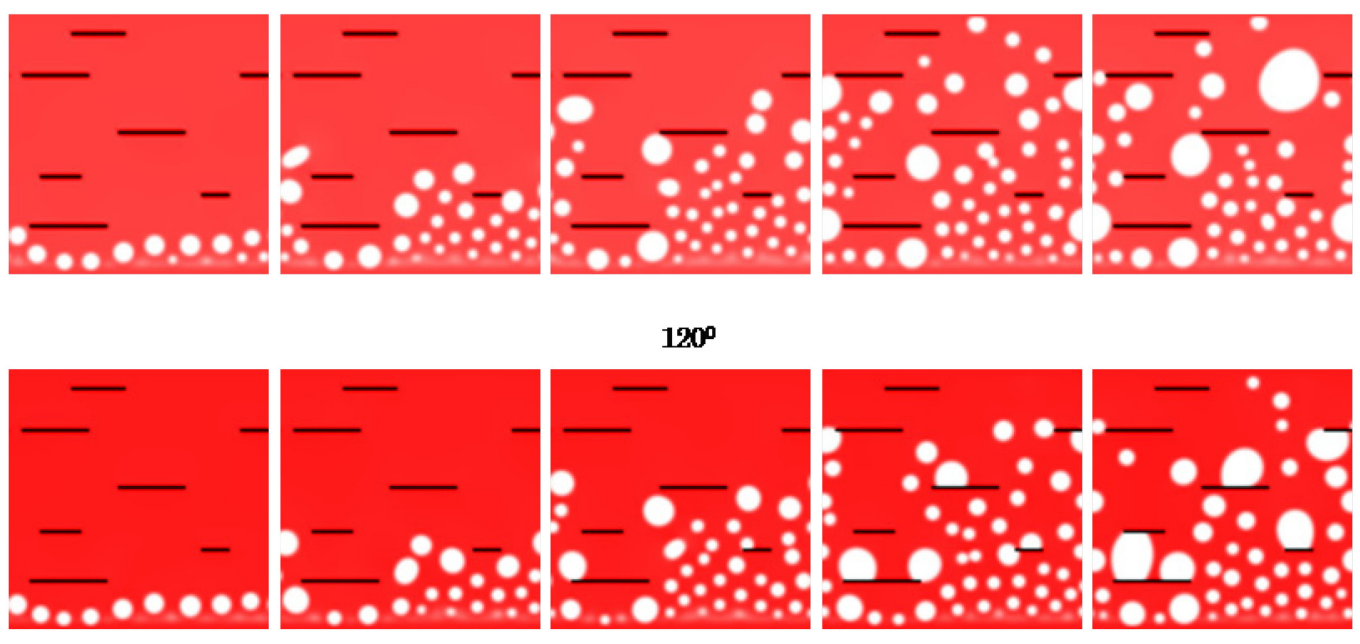

$120^{\circ}$

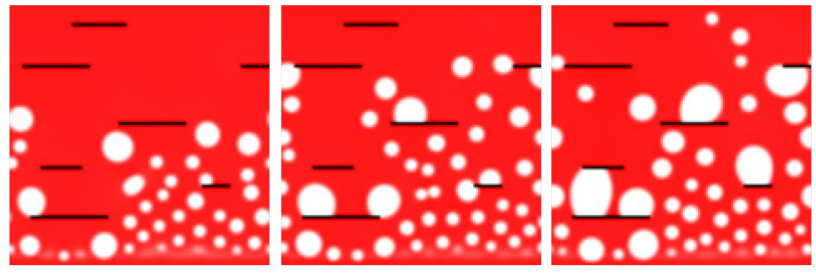

$90^{\circ}$
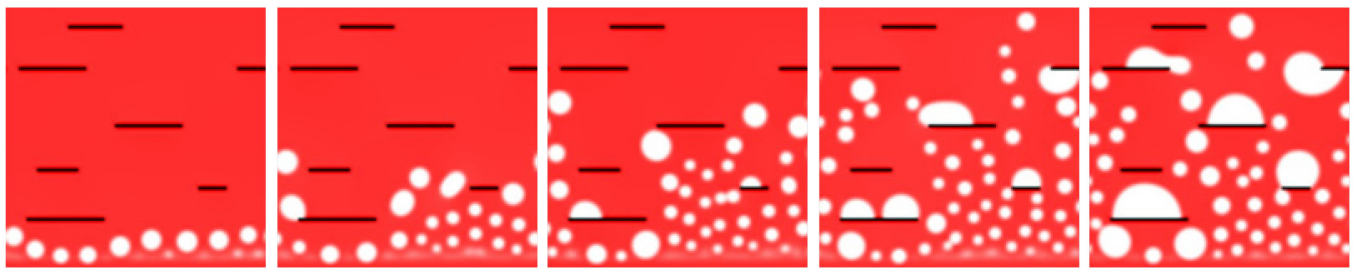

$15^{\circ}$
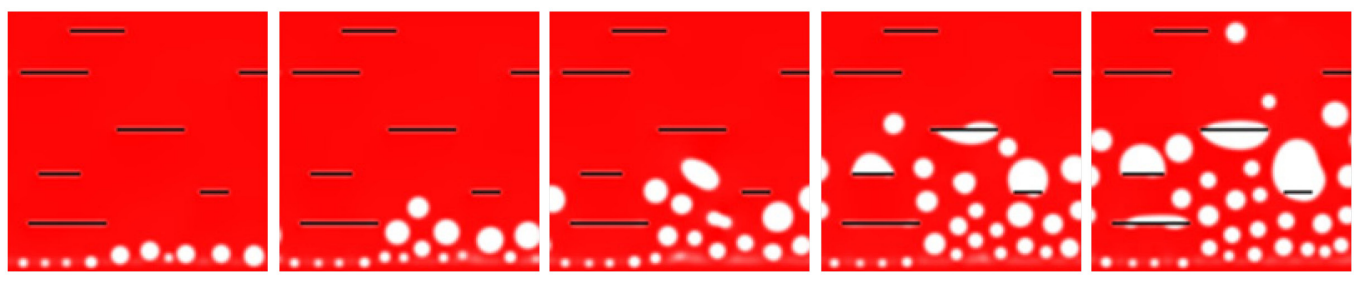

Figure 4.33. Multicomponent fluid flows interacting with solid surfaces for different contact angles. The white bubble is the wetting fluid. Simulation domain: 100×100 lattice nodes. Frames from left to right: 500, 1000, 1500, 2500, and 4000 time steps. From top to bottom: $180^{\circ}, 120^{\circ}, 90^{\circ}$, and $15^{\circ}$ contact angle. 


\section{Conclusion and Future Works}

Two dimensional simulations of single rising and multiple bubble dynamics using the multicomponent multiphase Shan and Chen-type Lattice Boltzmann method (LBM) have been presented. A new approach that uses effective buoyancy was proposed and proved to be consistent with the expected bubble deformation. Simulations results show that, as a results of buoyancy, a spherical bubble rises up and changes its shape based on Eötvös and Morton numbers. Bubbles deform from spherical to ellipsoidal shape and even to skirt-like structure at higher Eötvös numbers. These results agree very well with the shape regime chart proposed by Clift et al. [12] and Bhaga \& Weber [13]. The method has been validated against a no-break-up rising bubble benchmark case based on qualitative and quantitative analysis using the level set method. For high Eötvös numbers where the bubbles were observed to break up, the LBM simulations showed a better conservation of the bubble area (mass) relative to the Level Set method (LSM). The grid convergence study showed that the results from the LSM method can be improved by refining the grid size, though the computational cost increased dramatically as compared to the LBM. The LBM was also able to capture the circulation patterns in the flow field for cases at higher Re numbers. The wake flow circulation patterns obtained by our simulation agree well with other predictions as in [13] and [45]. 
Wall effects on rise velocity and final shape were also investigated and our simulations results fall into Clift's criterion [12]. Further cases for spherical cap bubbles $($ Eo $>40)$ were performed and our results matched very well with empirical predictions by Krishna [19]

Interaction and coalescence of multiple bubbles were also analyzed for different scenarios to better understand the dynamics and the coalescence characteristics. It was observed that in all configurations, the dynamics of the flow is dictated by the leading bubble, which deforms the most as a result of the drag from the surrounding liquid. The trailing bubble catches up with the leading bubble due to the wake left behind by the later. As a result, this reduces the trailing bubble drag and therefore allows the bubbles to coalesce. In order to check the vortex structure of the leading bubble on the trailing bubble, simulation of oblique coalescence was carried out and results compared favorably with 3 dimensional simulations by Annaland et al. [21].

Development and testing of gas generation source terms was also reported. Line and random source terms were implemented and multicomponent flow through porous media was investigated. Simulations for different contact angles were performed and results on interaction between fluids and solid surfaces due to contact angle and surface tension match the expectations.

From the simulation results presented here, it can be concluded that the current modeling method is suitable for and able to capture features in 
multicomponent flow simulations. The Lattice Boltzmann Method has proved to be a superior tool as compared to the Level Set Method in terms of mass conservation and computational efficiency.

My model gives detailed information that can be used to simulate bubbleinduced sediment mixing. At this stage, my model cannot simulate a solid particle phase. But a starting point will be to incorporate a third fluid component of higher effective density to mimic the solids and study a swarm of bubbles of the two different components, and then try to understand the transport of those solids.

Although successful results have been obtained with this two dimensional model, 3D effects need to be implemented to further investigate the capability of the Shan and Chen LBM. Three dimensional benchmark cases such as flow in a pipe and a rectangular duct are first steps toward the implementing of the $3 \mathrm{D}$ code, and results using parallel computing are presented in Appendix C. In addition, to assess the air-water flow regime, simulation of fluid flows in a low regime of Morton number need to be performed using parallel computing.

Because the Shan and Chen approach solves for low density ratio, a multirelaxation time collision operator coupled to the implicit LBM scheme [78] can be a starting point to investigate multiphase flows with high density ratio. 
In order to fully understand mechanisms that control the ebullition flux of methane in peat soils, future works will be directed towards ebullitiontriggered phenomena based on peat characteristics, atmospheric pressure, and other variables. In addition, experimental and field work will be performed in the near future and real peat structures from computed tomography and physically-appropriate parameters will be included in simulations to support the numerical efforts. 


\section{REFERENCES}

[1] J. Hua and J. Lou, "Numerical simulation of bubble rising in viscous liquid," J. Comput. Phys., vol. 222, no. 2, pp. 769-795, 2007.

[2] A. J. Baird, C. W. Beckwith, S. Waldron, and J. M. Waddington, "Ebullition of methane-containing gas bubbles from near-surface Sphagnum peat," Geophys. Res. Lett., vol. 31, pp. 1-4, 2004.

[3] T.J. Coulthard, A.J. Baird, J. Ramirez, and J.M. Waddington, "Methane Dynamics in Peat: Importance of Shallow Peats and a Novel ReducedComplexity Approach for Modeling Ebullition," Geophysical Monograph Series, vol. 184, pp. 173-185, 2009.

[4] G.M. King, T. Berman, and W.J. Wiebe, "Methane Formation in the Acidic Peats of Okefenokee Swamp, Georgia," American Midland Naturalist, vol. 105, no. 2, pp. 386-389., 1981.

[5] E.A. Daymo, "Industrial Mixing Techniques for Hanford Double-Shell Tanks.," PNNL- 11725 UC-72 1. Pacific Northwest National Laboratory, Richland, Washington., 1997.

[6] M.R. Powell, and C.R. Hymas, "Retrieval Process Development and Enhancements FY96 Pulsed-Air Mixer Testing and Deployment Study," Pacific Northwest National Laboratory, Richland, Washington 99352, PNNL-11200 UC-721, 1996.

[7] A. Gupta, and R. Kumar, "Lattice Boltzmann simulation to study multiple bubble dynamics," Int. J. Heat Mass Transfer, vol. 51, pp. 51925203, 2008.

[8] Inamuro, T., Ogata, T., and Ogino, F., "Numerical simulation of bubble flows by the Lattice Boltzmann method," Future Generation Computational Systems, vol. 20, pp. 959-954, 2004.

[9] H.W. Zheng, C. Shu, Y.T. Chew, "A lattice Boltzmann model for multiphase flows with large density ratio," J. Comput. Phys., vol. 218, pp. 353-371, 2006. 
[10] J. Tolke, M. Krafczyk, M. Schulz, and E. Rank, "Lattice Boltzmann simulations of binary fluid flow through porous media," Phil. Tans. R. soc. Lond. A, vol. 360, pp. 535-545, 2002.

[11] K. Sankaranarayanan, X. Shan, I.G. Kevrekidis, S. Sundaresan, "Bubble flow simulations with the lattice Boltzmann method," Chemical Engineering Science, vol. 54, pp. 4817-4823, 1999.

[12] R. Clift, J.R. Grace, and M.E. Weber, Bubbles, Drops and Particles. New York: Academic Press, 1978.

[13] D. Bhaga, M.E. Weber, "Bubbles in viscous liquids: shapes, wakes and velocities," J. Fluid Mech., pp. 61-85, 1981.

[14] Yu. B. Zudin, "Calculation of the rise velocity of large gas bubbles," J. Eng. Phys. Thermophys., vol. 68, no. 1, pp. 10-15, 1995.

[15] G. Bozzano and M. Dente, "Shape and terminal velocity of single bubble motion: a novel approach," Computers and Chemical Engineering, vol. 25, pp. 571-576, 2001.

[16] D.D. Joseph, "Rise velocity of a spherical cap bubble," J. Fluid Mech., vol. 488, pp. 213-223, 2003.

[17] R. M. Davies, and G. I. Taylor, "The mechanics of large bubbles rising through liquids in tubes.," Proc. R. Soc. Lond. A, vol. 200, pp. 375-390, 1950 .

[18] Y. Nakahara, Y. Adachi, S. Hosokawa A.Tomiyama, "Shape and rising velocities of single bubbles rising through an ineer subchannel," J. Nucl. Sci. Technology, vol. 40, no. 3, pp. 136-142, 2003.

[19] R. Krishna, M.I. Urseanu, J.M. van Baten and J. Ellenberger, "Wall effects on the rise of single gas bubbles in liquids.," Int. Comm. Heat Mass Transfer, vol. 26, no. 6, pp. 781-790, 1999.

[20] E. Olsson, G. Kreiss., "A conservative level set method for two phase 
flow.," J. Comput. Phys., vol. 210, pp. 225-246, 2005.

[21] M. van Sint Annaland, N.G. Deen, and J.A.M. Kuipers, "Numerical simulation of gas bubbles behaviour using a three-dimensional volume of fluid," Chemical Engineering Science, vol. 60, pp. 2999-3011, 2005.

[22] P. K. Volkov, "Dynamics of a liquid with gas bubbles," Fluid Dynamics, vol. 31, no. 3, pp. 399-409, 1996.

[23] T. Miyahara, S. Kaseno, and T. Takahashi, "Studies on Chains of Bubbles Rising Through Quiescent Liquid," The Canadian J. Chem. Eng., vol. 62, pp. 186-193, April 1984.

[24] A.C. Hoffmann and H.A. van den Bogaard, "A Numerical Investigation of Bubbles Rising at Intermediate Reynolds and Large Weber Numbers," Ind. Eng. Chem. Res., vol. 34, pp. 366-372, 1996.

[25] E. Delnoij, J.A.M. Kuipers, and W.P.M. van Swaaij, "Numerical simulationof bubble coalescence using volume of fluid (VOF) model," in Third International Conference on Multiphase Flow, ICMF'98, Lyon, France, 1998.

[26] M. Koebe, D. Bothe, and H.-J. Warnecke, "Direct numerical simulations of air bubbles in water/glycerol mixtures: shapes and velocity fields," in 4th ASME-JSME Joint Fluids Enginnering Conference, Honolulu, Hawaii, USA, 2003.

[27] M. van Sint Annaland, W. Dijkhuizen, N.G. Deen, and J.A.M. Kuipers, "Numerical Simulation of Behavior of Gas Bubbles Using a 3-D FrontTracking Method," AIChE Journal, vol. 52, no. 1, January 2006.

[28] A.K. Gunstensen, D.H. Rothman, and S. Zaleski, "Lattice Boltzmann model of immiscible fluids.," Phys. Rev. A, vol. 43, no. 18, pp. 4320-4327, 1991.

[29] X. Shan, and H. Chen, "Lattice Boltzmann model for simulating flows with multiple phases and components," Phys. Rev. E, vol. 47, no. 3, pp. 1815-1819, 1993. 
[30] M.R. Swift, W.R. Osborn, and J.M. Yeomans, "Lattice Boltzmann Simulation of Nonideal Fluids," Phys. Rev. Lett., vol. 74, no. 5, pp. 830833, 1995.

[31] X. He, X. Shan, and G.D. Doolen, "Discrete Boltzmann equation model for nonideal gases," Phys. Rev. E, vol. 57, no. 1, pp. 13-16, 1998.

[32] D.H. Rothman, and J.M. Keller, "Immiscible cellular-automaton fluids," J. Statistical Phys., vol. 52, no. 3-4, pp. 1119-27, 1988.

[33] D. Grunau, S. Chen, and K. Eggert, "A lattice Boltzmann model for multiphase fluid flows," Physics of Fluids A, vol. 5, no. 10, pp. 2557-62, 1993.

[34] H. Huang, D.T. Thorne, M.G. Schaap, and M.C. Sukop, "Proposed approximation for contact angles in the Shan-and-Chen-type multicomponent multiphase lattice Boltzmann models," Phys. Rev. E, vol. 76, no. 066701, pp. 1-6, 2007.

[35] P. Yuan and L. Schaefer, "Equations of state in a lattice Boltzmann model," Phys. Fluids, vol. 18, pp. 042101-, 2006.

[36] S. Chibbaro, G. Falcucci, G. Chiatti, H. Chen, X. Shan, S. Succi, "Lattice Boltzmann models for nonideal fluids with arrested phase-separation," Phys. Rev. E, vol. 77, no. 3, pp. 036705-, 2008.

[37] Z. Yu, L-S. Fan, "Multirelaxation-time interaction-potential-based lattice Boltzmann model for two-phase flow," Phys. Rev. E, vol. 82, p. 046708, 2010.

[38] C.M. Pooley and K. Furtado, "Eliminating spurious velocities in the freeenergy lattice Boltzmann method.," Phys. Rev. E, vol. 77, no. 046702, pp. 1-9, 2008.

[39] T. Inamuro, T. Ogata, S. Tajima, and N. Konishi, "A lattice Boltzmann method for incompressible two-phase flows with large density differences," J. Comp. Phys, vol. 198, pp. 628-644, 2004. 
[40] K. Sankaranarayanan, I.G. Kevrekidis, S. Sundaresan, J. Lu, G. Tryggvason, "A comparative study of lattice Boltzmann and fronttracking finite-difference methods for bubble simulations," Int. J. Mult. Phase Flow, vol. 29, pp. 109-116, 2003.

[41] N. Takada, M. Misawa, A. Tomiyama, and S. Hosokawa., "Simulation of bubble motion under gravity by Lattice Boltzmann method," J. Nucl. Sci. Technol., vol. 38, no. 5, pp. 330-341, 2001.

[42] X. Frank, D.Funfschilling, N.L. Midoux, and H.Z. Li, "Bubbles in a viscous liquid: lattice Boltzmann simulation and experimental validation.," J. Fluid Mech., vol. 545, pp. 113-122, 2006.

[43] I.O. Kurtoglu, and C.-L. Lin, "Lattice Boltzmann study of bubble dynamics.," Num. Heat Transfer Part B, vol. 50, pp. 333-351, 2006.

[44] G. Thömmes, J. Becker, M. Junk, A.K. Vaikuntam, D. Kehrwald, A. Klar, K. Steiner, A. Wiegmann, "A lattice Boltzmann method for immiscible multiphase flow simulations using the level set method," J. Comput. Phys., vol. 228, pp. 1139-1156, 2009.

[45] L. Amaya-Bower and T. Lee., "Single bubble rising dynamics for moderate Reynolds number using Lattice Boltzmann Method. ," Computers \& Fluids , vol. 39, pp. 1191-1207, 2010.

[46] H. Huang, H. Zheng, X-Y. Lu and C. Shu., "An evaluation of a 3D freeenergy-based lattice Boltzmann model for multiphase flows with large density ratio.," Int. J. Numer. Meth. Fluids., vol. 63, pp. 1193-1207, 2010.

[47] A. Fakhari and M. H. Rahimian, "Simulation of an axisymmetric rising bubble by a multiple relaxation time lattice Boltzmann method.," Int. J. Modern Phys. B, vol. 23, no. 21, pp. 4907-4932, 2009.

[48] J. Chao, R. Mei, R. Singh, and W. Shyy, "A filter-based, mass-conserving lattice Boltzmann method for immiscible multiphase flows.," Int. J. Numer. Meth. Fluids, vol. DOI: 10.1002/fld.2276, 2010.

[49] S. Wolfram, "Cellular automaton automaton fluid 1: Basic theory.," $J$. 
Stat. Phys., vol. 45, pp. 471-526, 1986.

[50] T. Inamuro, M. Yoshino, and F. Ogino, "A non-slip boundary condition for lattice Boltzmann simulations," Phys. Fluids, vol. 7, no. 12, pp. 29282930, 1995.

[51] D.R. Noble, J.G. Georgiadis, and R.O. Buckius, "Direct Assessment of Lattice Boltzmann Hydrodynamics and Boundary Conditions for Recirculating Flows," J. Stat. Phys., vol. 81, no. 1-2, pp. 17-33, 1995.

[52] I. Ginzbourg and D. d'Humieres, "Local Second-Order Boundary Methods for Lattice Boitzmann Models," J. Stat. Phys., vol. 84, no. (5-6), pp. 927971, 1996.

[53] R.S. Maier, R.S. Bernard, and D.W. Grunau, "Boundary conditions for the lattice Boltzman nmethod," Phys. Fluids, vol. 8, pp. 1788-1801, 1996.

4] S. Chen, D. Martinez, and R. Mei, "On boundary conditions in lattice Boltzmann methods," Phys. Fluids, vol. 8, no. 9, pp. 2527-2536, 1996.

[55] X. He and Q. Zou, "On pressure and velocity boundary conditions for the lattice Boltzmann BGK model," Phys. Fluids, vol. 9, no. 6, pp. 1591-1598, 1997.

[56] R. Mei, L.-S. Luo, and W. Shyy, "An Accurate Curved Boundary Treatment in the Lattice Boltzmann Method," J. Comput. Phys., vol. 155, pp. 307-330, 1999.

[57] R. Mei, W. Shyy, Dazhi Yu, and L.-S. Luo, "Lattice Boltzmann Method for 3-D Flows with Curved Boundary," J. Comput. Phys., vol. 161, pp. 680-699, 2000.

[58] U. Frisch, B. Hasslacher, and Y. Pomeau, "Lattice-gas automata for the Navier-Stokes equation.," Phys. Rev. Lett., vol. 56, no. 14, pp. 1505-1508, 1986.

[59] S. Succi, The lattice Boltzmann equation for fluid dynamics and beyond.: 
Oxford Science Publications, 2001.

[60] G.R. McNamara and G. Zanetti , "Use of the Boltzmann Equation to Simulate Lattice-Gas Automata," Phys. Rev. Lett., vol. 61, no. 20, pp. 2332-2335, 1988.

[61] P.L. Bhatnagar, E.P. Gross, and M. Krook, "A Model for Collision Processes in Gases. I. Small Amplitude Processes in Charged and Neutral One-Component Systems," Phys. Rev. , vol. 94, pp. 511-525, 1954.

[62] Michael C. Sukop, and Daniel T. Thorne, Lattice Boltzmann Modeling: An Introduction for Geoscientists and Engineers. Berlin: Springer, 2006.

[63] A. Mezrhab, M. A. Moussaoui, M. Jami, H. Naji, and M. Bouzidi, "Double MRT thermal lattice Boltzmann method for simulating convective flows," Phys. Letters A, vol. 374, pp. 3499-3507, 2010.

[64] T. Lee, C.-L. Lin, "A stable discretization of the lattice Boltzmann equation for simulation of incompressible two-phase flows at high density ratio," J. Comput. Phys., vol. 206, pp. 16-47, 2006.

[65] N.S. Martys and H. Chen, "Simulations of multicomponent fluids in complex three-dimensional geometry by the lattice Boltzmann method," Phys. Rev. E, vol. 53, no. 1, pp. 743-751, 1996.

[66] X. He, and G.D. Doolen, "Thermodynamic Foundations of Kinetic Theory and Lattice Boltzmann Models for Multiphase Flows," J. Stat. Phys., vol. 107, no. 1-2, pp. 309-328, 2002.

[67] X. Shan, "Analysis and reduction of spurious currents in a class of multiphase lattice Boltzmann models," Phys. Rev. E, vol. 73, no. 047701, pp. 1-3, 2006.

[68] COMSOL Multiphysics Modeling Guide, 2008, v. 3.5a.

[69] S. Hysing, "Numerical Simulation of Immiscible Fluids with FEM Level Set Techniques," Technical University of Dortmund, Germany, PhD 
2007.

[70] S. Hysing, S. Turek, D. Kuzmin, N. Parolini, E. Burman, S. Ganesan, and L. Tobiska, "Proposal for quantitative benchmark computations of bubble dynamics," Int. J. Num. Meth. Fluids, vol. 60, p. 1259, 2008.

[71] S. Hou, X. Shan, Q. Zou, G.D. Doolen, and W.E. Soll, "Evaluation of two lattice Boltzmann models for multiphase flows," J. Comput. Phys., vol. 138, pp. 695-713, 1997.

[72] G. Brereton, and D. Korotney, "Coaxial and oblique coalescence of two rising bubbles," in Dynamics of Bubbles and Vortices Near a Free Surface, AMD-vol. 119. New York: ASME, 1991.

[73] M.C. Sukop and D. Or, "Lattice Boltzmann method for modeling liquidvapor interface configurations in porous media," Water Ressources Research, vol. 40, pp. W01509 (1-11), 2004.

[74] E. Kellner, A.J. Baird, M. Oosterwoud, K. Harrison, and J.M. Waddington, "Effect of temperature and atmospheric pressure on methane (CH4) ebullition from near-surface peats," Geophys. Res. Lett., vol. 33, no. L18405, pp. 1-5, 2006.

[75] M. Strack, E. Kellner and J.M. Waddington, "Effect of entrapped gas on peatland surface level fluctuations," Hydrol. Process., vol. 20, pp. 3611$3622,2006$.

[76] T. Tokida T. Miyazaki, M. Mizoguchi, O. Nagata, F. Takakai, A. Kagemoto, and R. Hatano, "Falling atmospheric pressure as a trigger for methane ebullition from peatland," Global Biogeochem. Cycles, vol. 21, no. GB2003, pp. 1-5, 2007.

[77] A.F. Stalder, G. Kulik, D. Sage, L. Barbieri, P. Hoffmann , "A SnakeBased Approach to Accurate Determination of Both Contact Points and Contact Angles ," Colloids And Surfaces A, vol. 286, no. 1-3, pp. 92-103, September 2006.

[78] K. Sankaranarayanan, X. Shan, I.G. Kevrekidis, and S. Sundaresan, "Analysis of drag and virtual mass forces in bubbly suspensions using an 
implicit formulation of the lattice Boltzmann method," J. Fluid Mech., vol. 452, pp. 61-96, 2002.

[79] I. Tosun, D. Uner, and C. Ozgen, "Critical Reynolds number for Newtonian flow in rectangular ducts," Ind. Eng. Chem. Res, pp. 19551957, 1988.

[80] A.F. Stalder, T. Melchior, M. Müller, D. Sage, T. Blu, M. Unser , "LowBond Axisymmetric Drop Shape Analysis for Surface Tension and Contact Angle Measurements of Sessile Drops ," Colloids and Surfaces A , vol. 364, no. 1-3, pp. 72-81, July 2010. 


\section{APPENDICES}

\section{APPENDIX A: MATLAB SCRIPTS USED FOR POST PROCESSING in}

\section{LBM.}

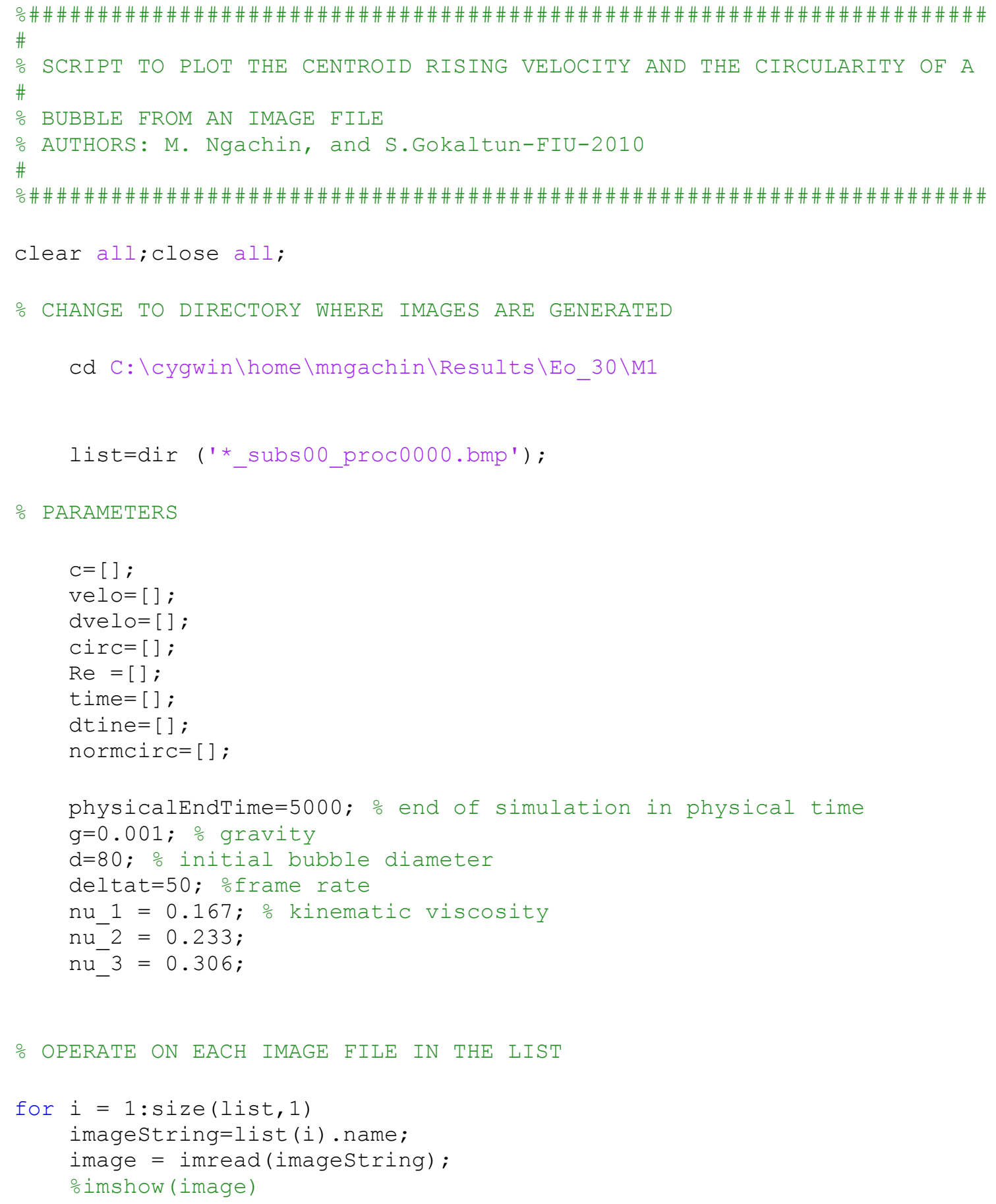









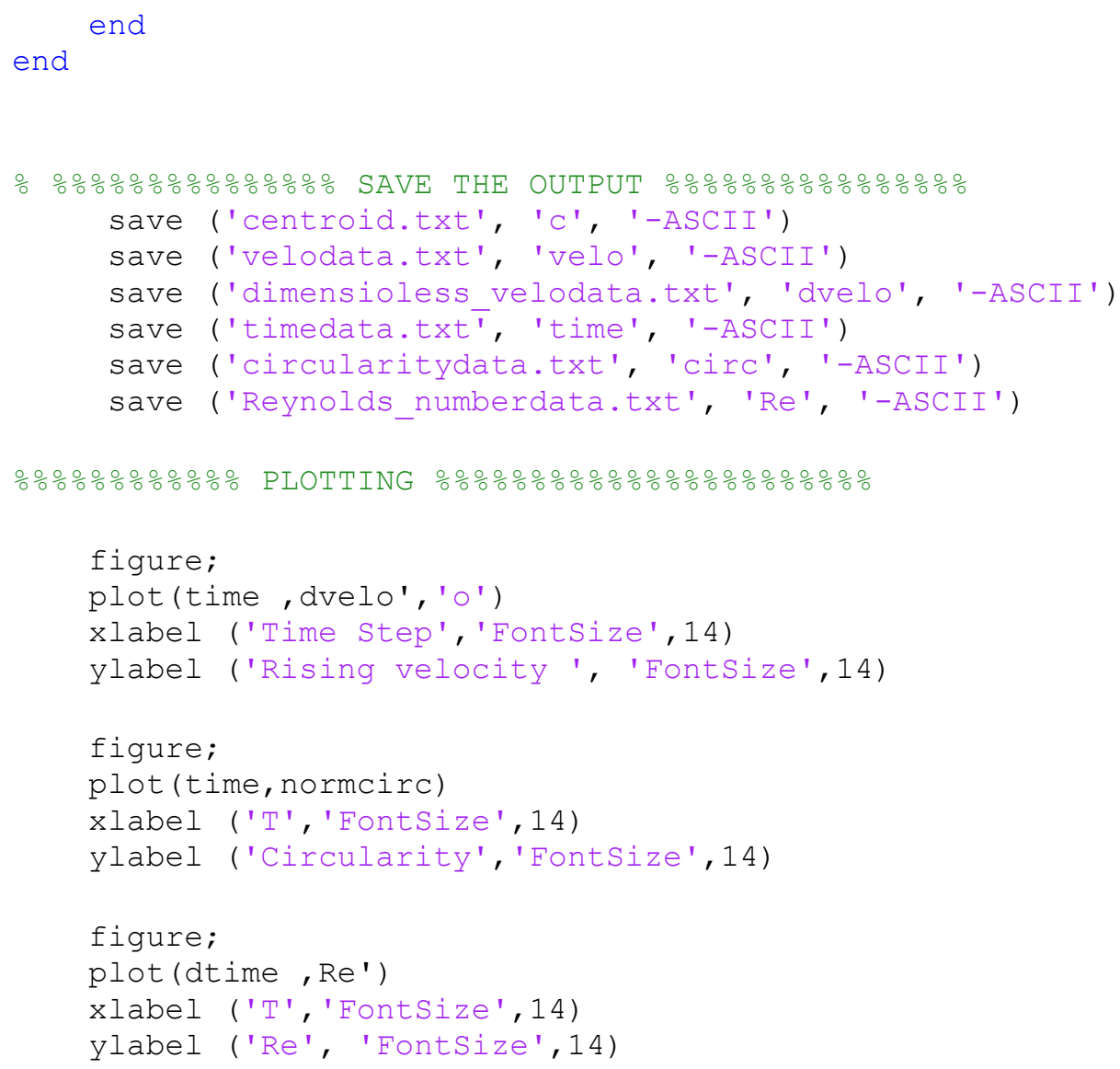









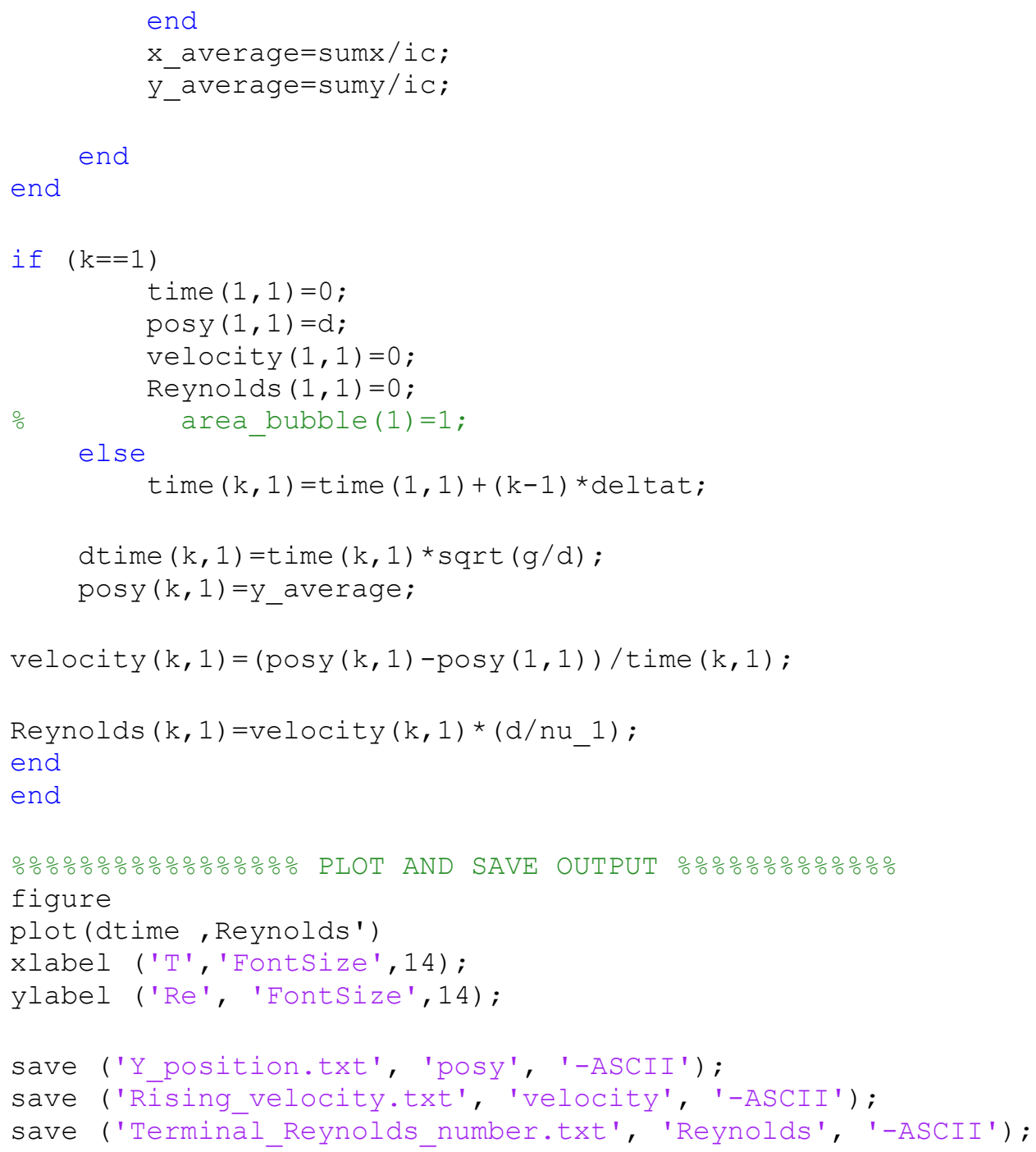














\section{APPENDIX B: PARAMETER FILES AND PSEUDO CODE USED TO GENERATE LINE AND RANDOM SOURCE TERMS}






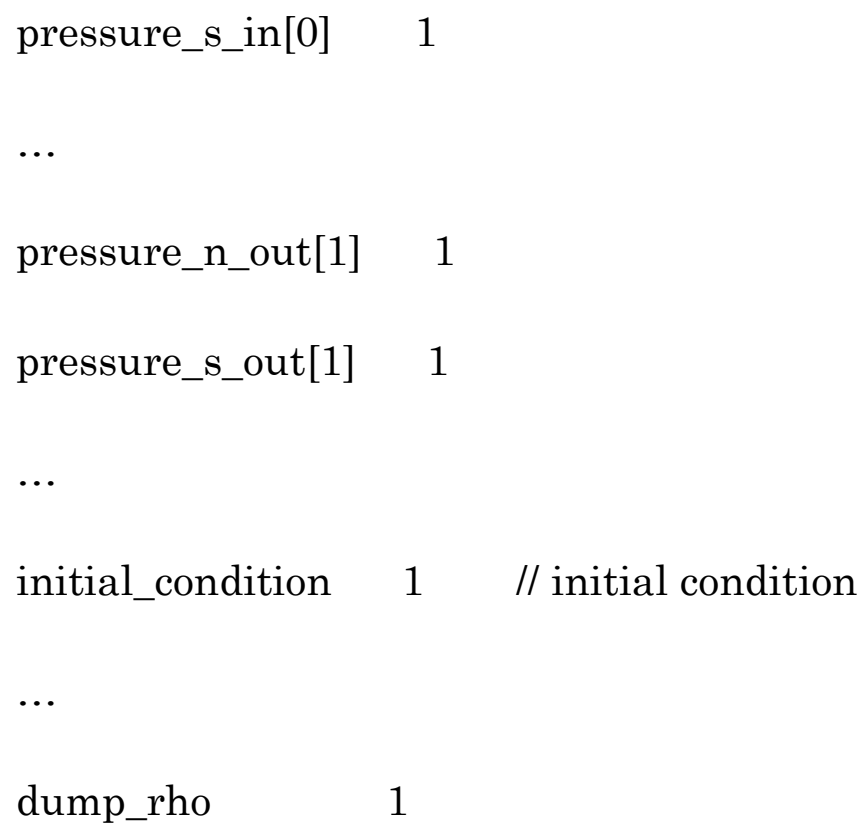

\section{Pseudo code implementing the line and the random source terms}






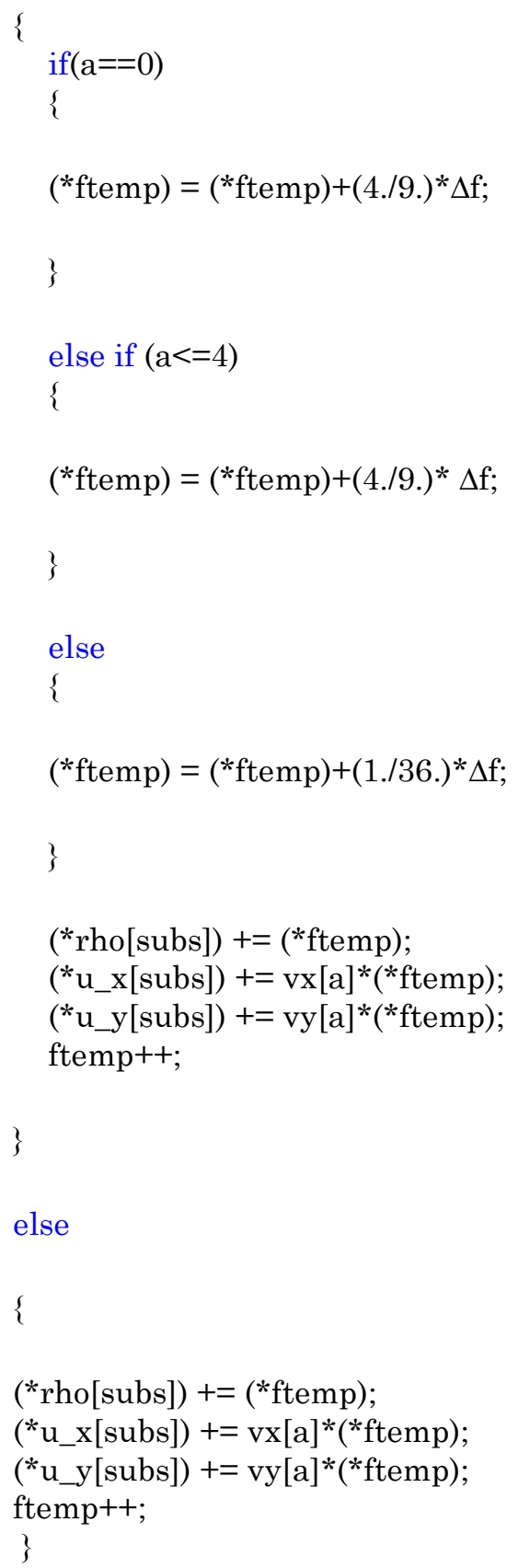




\section{APPENDIX C. FLOW IN A PIPE AND A RECTANGULAR DUCT}

To investigate the capability of our code to simulate $3 \mathrm{D}$ problems, and using parallel computing, flows in a pipe and rectangular duct were simulated and results were compare to analytical solution.

\section{Flow in a pipe}

A gravity-driven flow in a $21 \times 21 \times 21$ lu domain was simulated for 20,000 time steps, using serial and parallel computing. The domain was created using Microsoft Paint to create a single slice, which was then replicated. After the slices are created, we use ImageJ software to import the image sequence and combine them to create a $21 \times 21 \times 21$.raw pipe as indicated on Fig. C1.

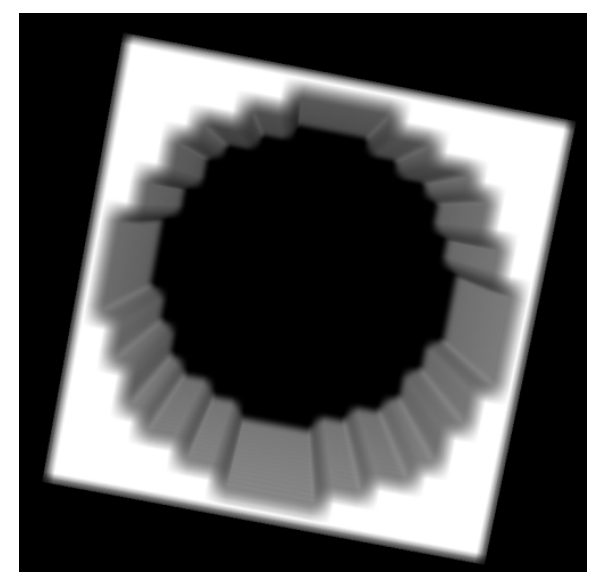

Figure C1: $21 \times 21 \times 21$ lattice nodes domain used for the simulation.

Simulation parameters include: the gravity acceleration $\mathrm{g}=0.001$, the kinematic viscosity $v=0.167 \mathrm{lu}^{2} \mathrm{ts}^{-1}$, the characteristic length $=19 \mathrm{lu}$ (pipe 
radius $\mathrm{r}=9.5 \mathrm{lu}$ ). Twenty one $(21)$ processors as indicated on line 5 in the bash script were used to run this case on a parallel machine:

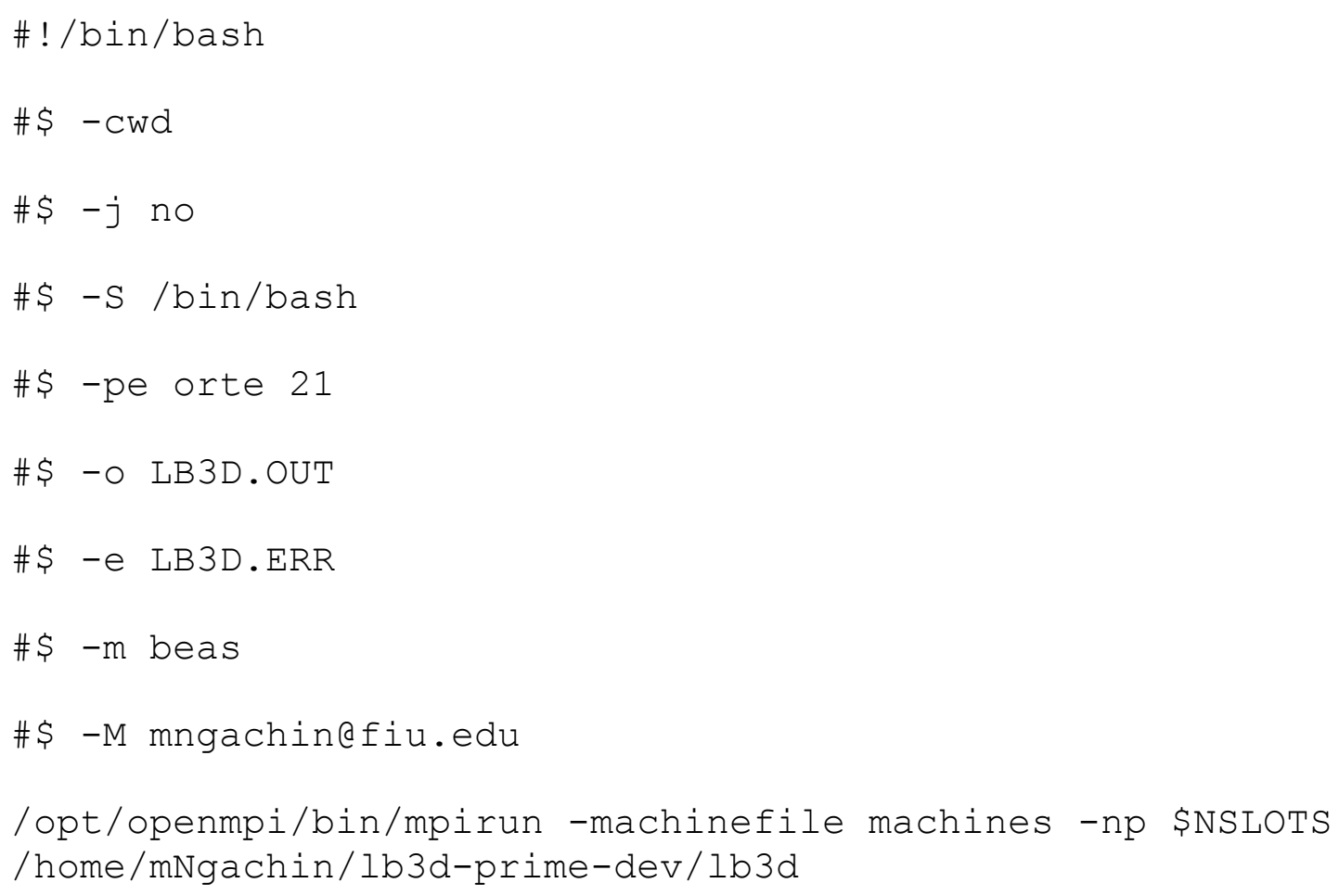

In order to check the domain partitioning in the parallel computer, we compare our results with serial computing. Both methods gave the same results, indicating that the partitioning works perfectly.

The velocity profile in Fig. C2 shows a maximum velocity of $0.13 \mathrm{lu} \mathrm{ts}^{-1}$ (average $=0.065 \mathrm{lu} \mathrm{ts}^{-1}$ ) in LBM. A comparison with the analytical value of $0.135 \mathrm{lu} \mathrm{ts}^{-1}$ (average $\bar{u}=g \frac{r^{2}}{8 v}=0.0675 \mathrm{lu} \mathrm{ts}^{-1}$ ) indicates a relative difference of $3.7 \%$, which is good considering the coarse discretization of the pipe 


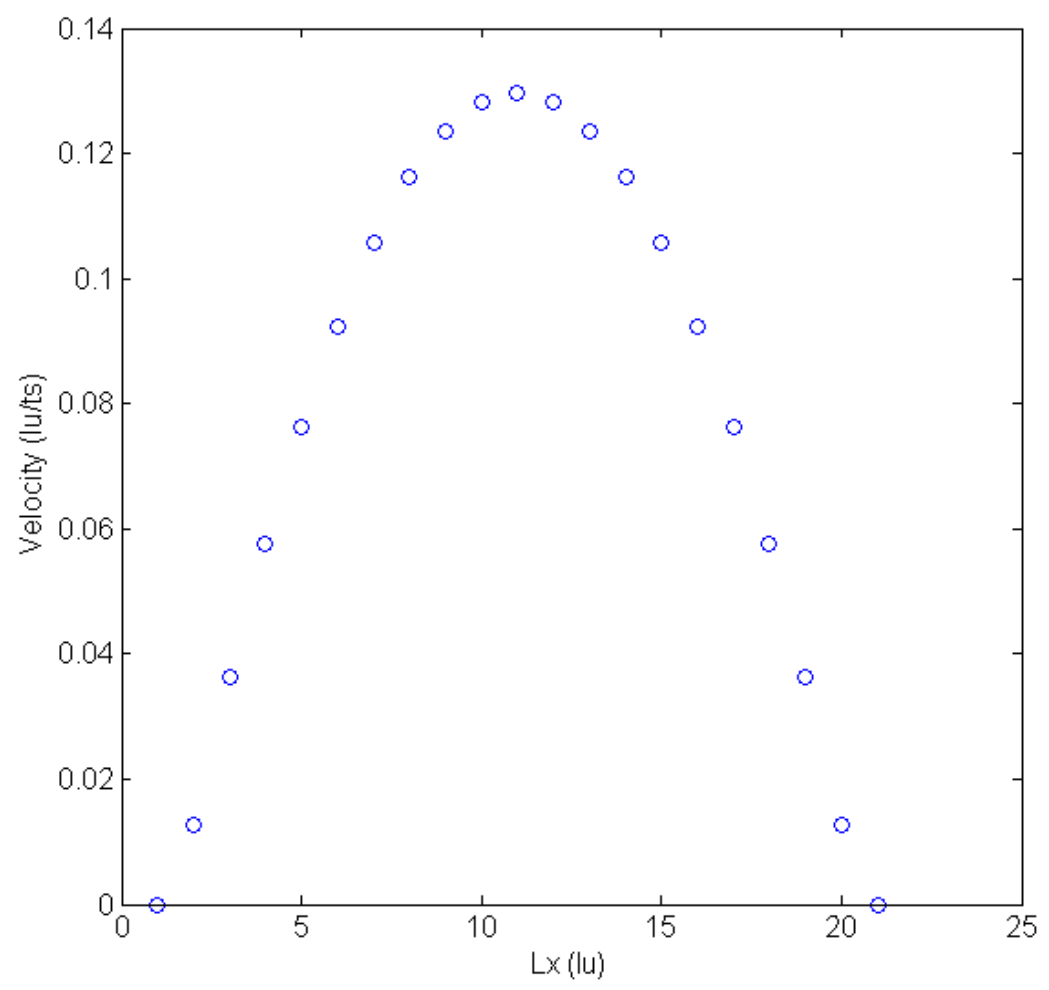

Figure C2: Velocity profile on a cross section along horizontal centerline obtained with parallel computing on a $21 \times 21 \times 21$ lattice nodes domain for 20,000 time steps.

\section{Flow in a square duct}

As previously, we consider a gravity-driven flow in a square duct with aspect ratio, $\alpha=1$, as shown on Fig C3. The gravity $\mathrm{g}=10^{-3} \mathrm{lu} \mathrm{ts}^{-2}$ and $v=0.167 \mathrm{lu}^{2} \mathrm{ts}^{-}$ 1 


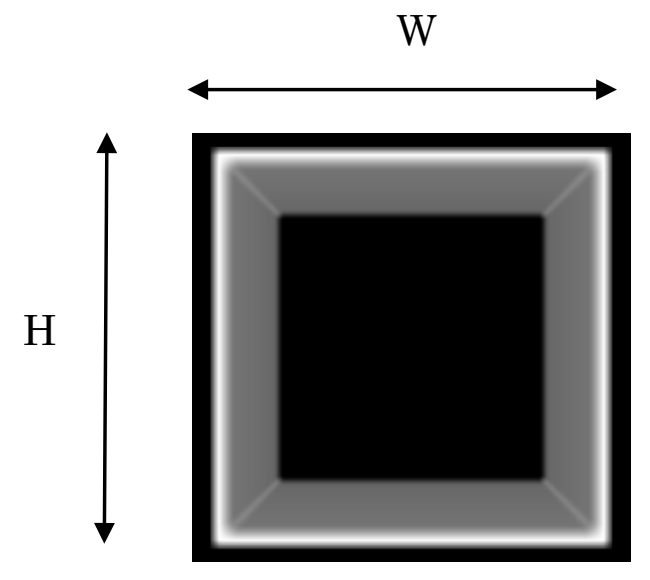

Figure C3: $23 \times 23 \times 23$ lattice nodes square duct (aspect ratio, $\alpha=\frac{H}{W}=1$ )

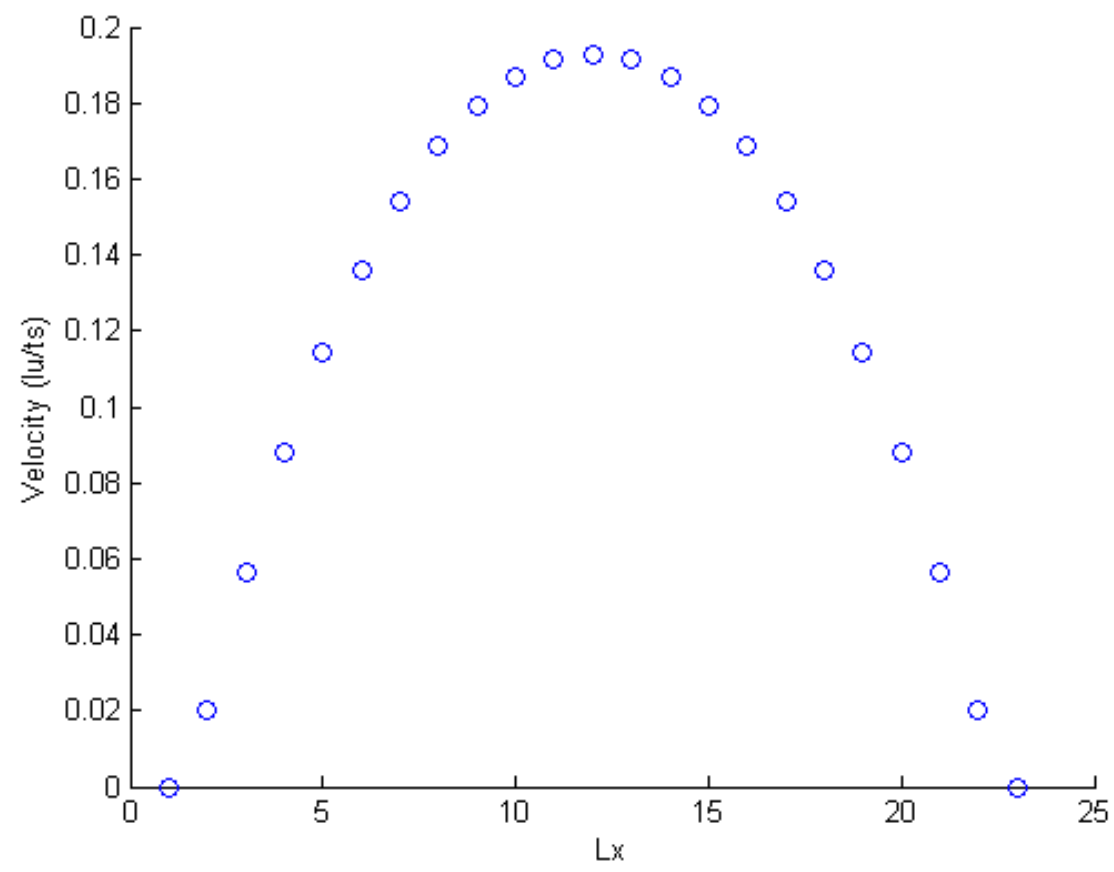

Figure C4: Velocity profile in a square duct obtained with parallel computer; on a $23 \times 23 \times 23$ lu domain for 10,000 time steps.

Figure $\mathrm{C} 4$ gives the velocity profile. The average velocity for flow in a square duct is defined as 


$$
\bar{u}=F \frac{a^{2}}{3 \mu} Y=g \frac{a^{2}}{3 v} Y
$$

where $F$ is the driving force, and the function $Y=1-\frac{192}{\pi^{5}}\left(\frac{a}{b}\right) \sum_{j=1}^{\infty} \frac{1}{(2 j-1)} \tanh \left[\frac{(2 j-1) \pi b}{2 a}\right]$. For aspect ratio $=1, Y=0.422[79]$.

The computed average velocity is $0.0928 \mathrm{lu} \mathrm{ts}^{-1}$ as compared with the analytical average of $0.093 \mathrm{lu} \mathrm{ts}^{-1}$, indicating a relative difference of $0.2 \%$. 


\section{APPENDIX D: PLOTS OF TERMINAL REYNOLDS NUMBERS OF THE DIFFERENT CASES PRESENTED IN THIS WORK.}
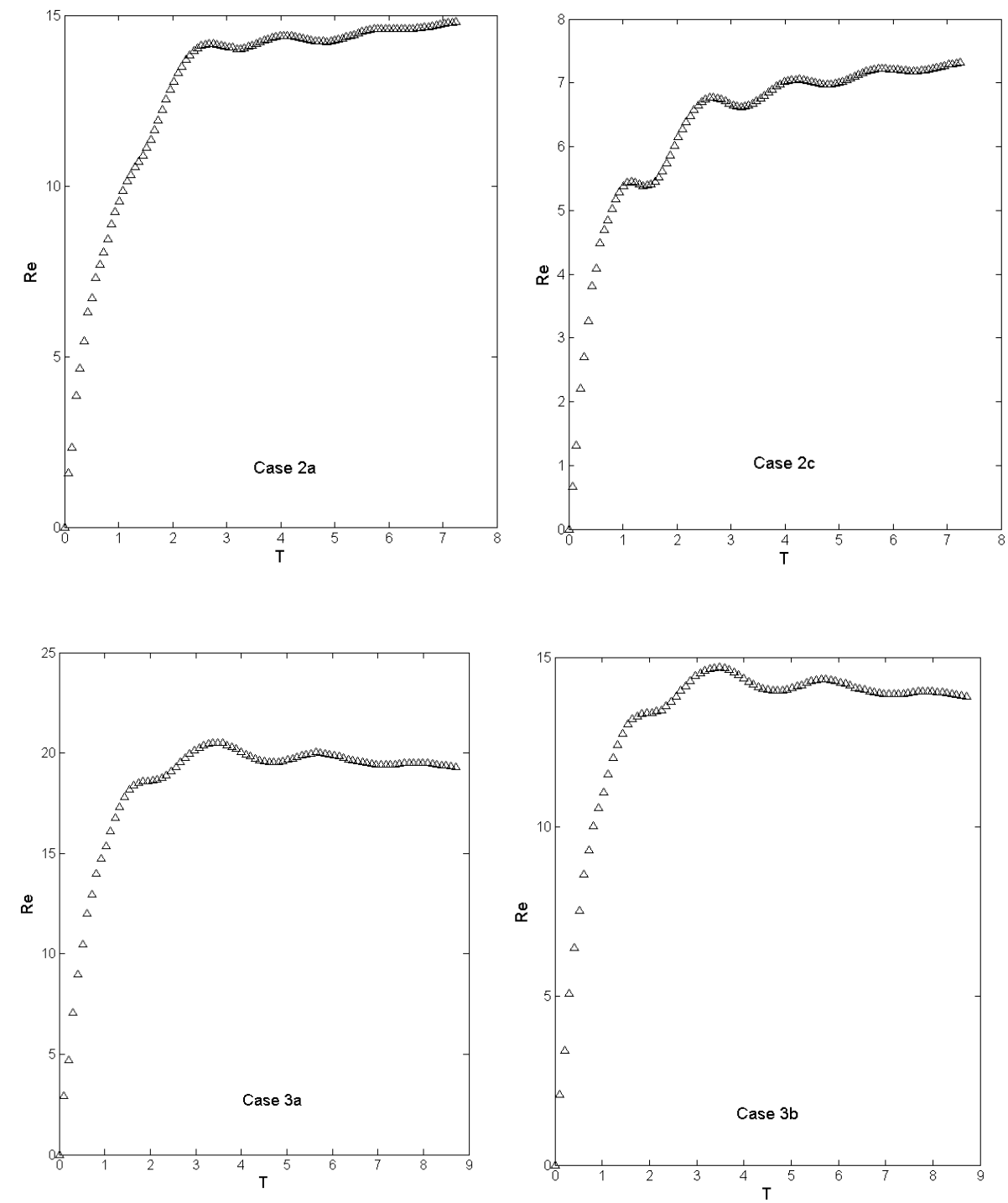

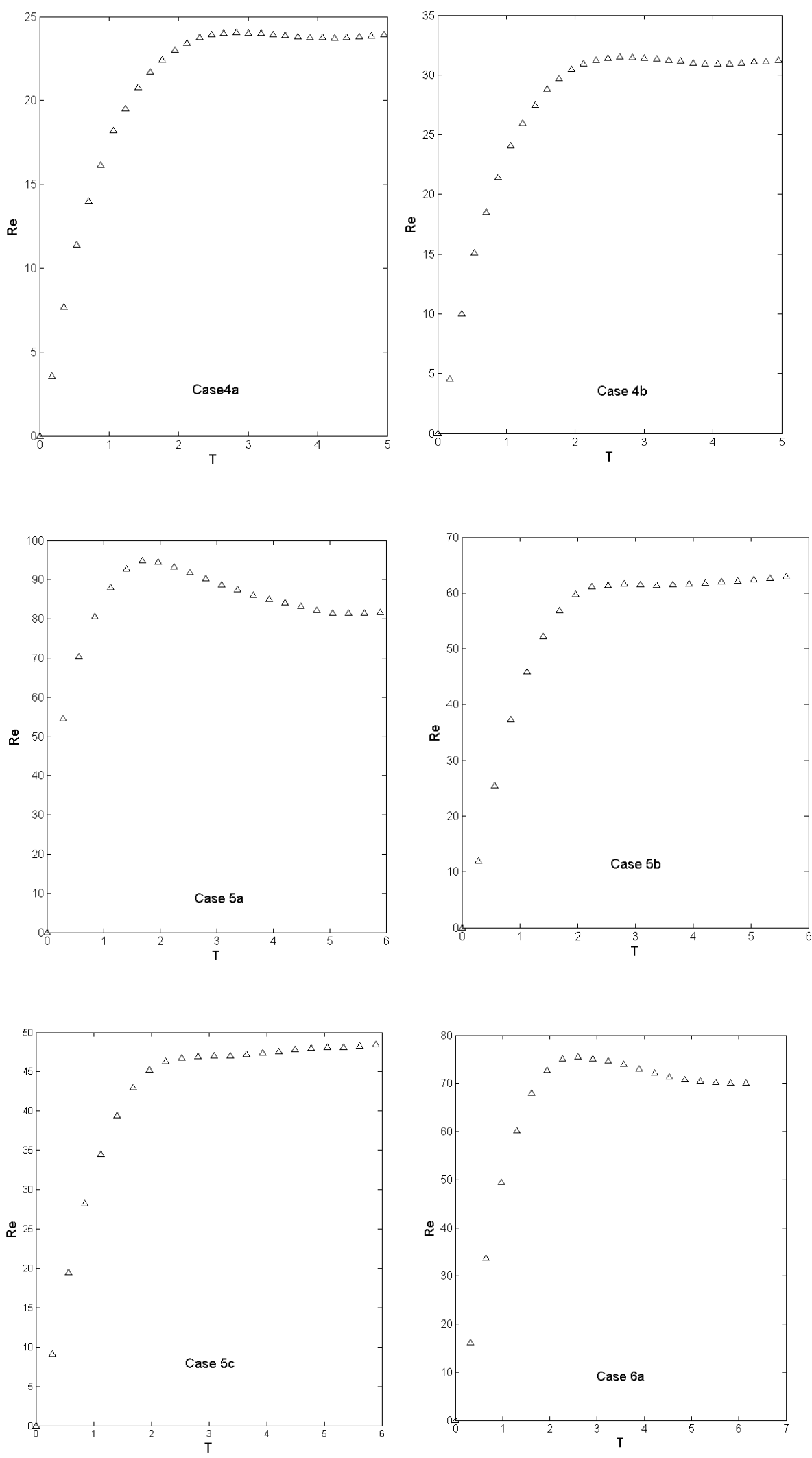


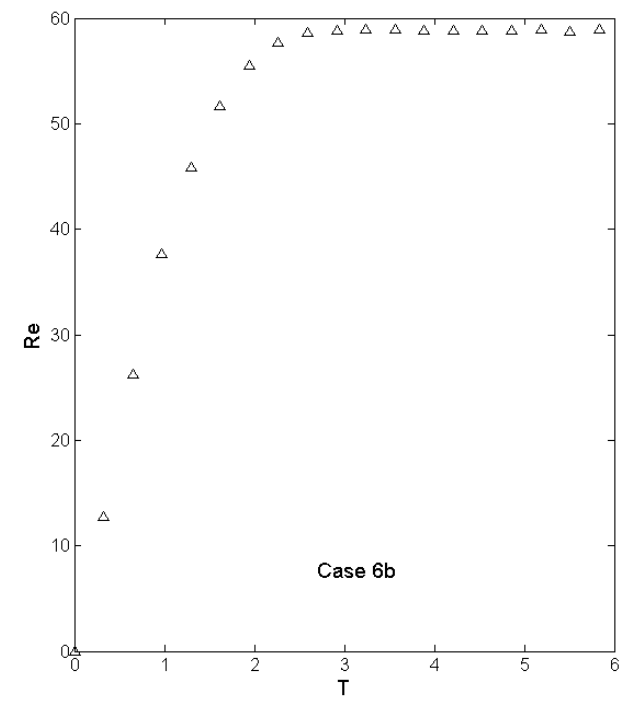




\section{APPENDIX E: CONTACT ANGLE MEASUREMENT USING LOW BOND AXISYMMETRIC DROP SHAPE ALGORITHM (LB-ADSA) UNDER IMAGEJ SOFTWARE.}

\section{Installation}

Get ImageJ and download the plugin drop_analysis.zip (www.bigwww.epfl.ch/demo/dropanalysis). Extract drop_analysis.zip in the "plugins" folder of ImageJ and extract all the files in a new folder "drop_analysis".

\section{How to use}

Open the image and make sure the image is in grayscale. On the "Process" menu of Image J, go under "Binary" and then on "Make Binary" as in Figure E1 below.

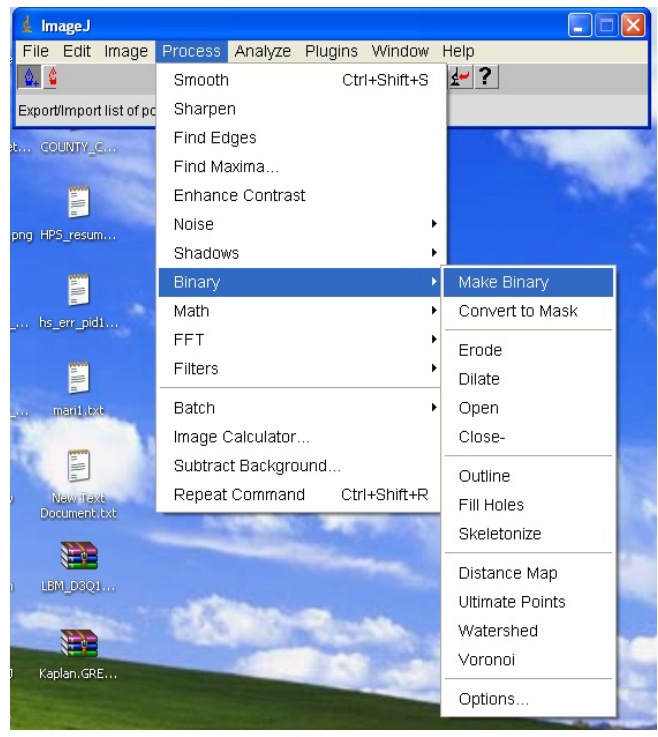

Figure E1. 
In the "plugins" menu go under "Drop Analysis-LB-ADSA". Then adjust the drop parameters until you completely recover the drop.

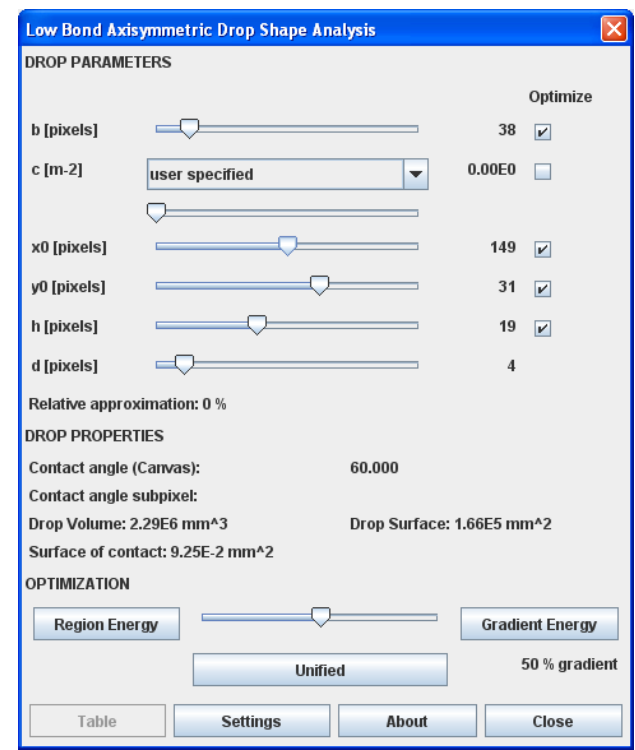

Figure E2. Low Bond Axisymmetric Drop Shape Analysis (LB-ADSA) indows.

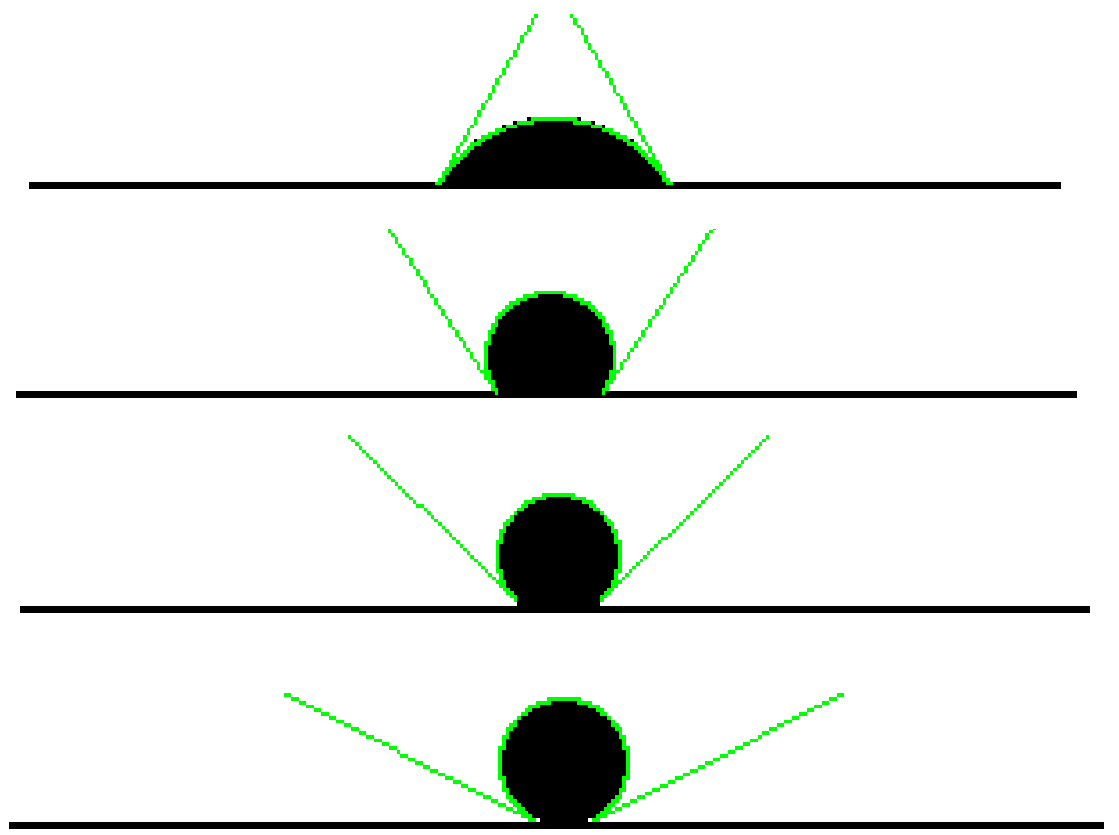

Figure E3. From top to bottom, snapshot of $60^{\circ} 120^{\circ}, 135^{\circ}$, and $150^{\circ}$ contact angle measured using the LB-ADSA algorithm [80]. The green line perfectly recovers the bubble shape obtained with LBM. 